\title{
Pitch Angle Control for a Small-Scale \\ Darrieus Vertical Axis Wind Turbine with Straight Blades (H-type VAWT)
}

by

Gebreel Abdalrahman

\author{
A thesis \\ presented to the University of Waterloo \\ in fulfillment of the \\ thesis requirement for the degree of \\ Doctor of Philosophy \\ in \\ Mechanical and Mechatronics Engineering
}

Waterloo, Ontario, Canada, 2019

(C) Gebreel Abdalrahman 2019 


\section{EXAMINING COMMITTEE MEMBERSHIP}

The following served on the Examining Committee for this thesis. The decision of the Examining Committee is by majority vote.

External Examiner

Supervisor(s)

Internal Member

Internal Member

Internal-external Member
Professor. Simon Yang

School of Engineering

University of Guelph, Guelph, Canada

Professor. Fue-Sang Lien \& Professor. William Melek

Department of Mechanical \& Mechatronic Engineering

University of Waterloo, Waterloo, Canada

Professor. John Wen

Department of Mechanical \& Mechatronic Engineering

University of Waterloo, Waterloo, Canada

Professor. Soo Jeon

Department of Mechanical \& Mechatronic Engineering

University of Waterloo, Waterloo, Canada

Professor. Fakhreddine Karray

Department of Electrical and Computer Engineering

University of Waterloo, Waterloo, Canada 


\section{DECLARATION}

I hereby declare that I am the sole author of this thesis. This is a true copy of the thesis, including any required final revisions, as accepted by my examiners.

I understand that my thesis may be made electronically available to the public. 


\section{Abstract}

Unlike the horizontal axis wind turbines, only a few studies have been conducted recently to improve the performance of a Darrieus Vertical Axis Wind Turbine with straight blades (H-type VAWT). Pitch angle control technique is used to enhance the performance of an $\mathrm{H}$ type VAWT in terms of power output and self-starting capability. This thesis aims to investigate the performance of an H-type VAWT using an intelligent blade pitch control system. Computational Fluid Dynamics (CFD) is used to determine the optimum pitch angles and study their effects on the aerodynamic performance of a 2D H-type VAWT at different Tip Speed Ratios (TSRs) by calculating the power coefficient $\left(C_{p}\right)$. The results obtained from the CFD model are used to construct the aerodynamic model of an H-type VAWT rotor, which is required to design an intelligent pitch angle controller based on Multi-Layer Perceptron Artificial Neural Networks (MLP-ANN) method. The performance of the blade pitch controller is investigated by adding a conventional controller (PID) to the MLP-ANN controller (i.e., Hybrid controller). For stability analysis, an H-type VAWT is modeled in nonlinear state space by determining the mathematical models for an H-type VAWT components along with Hybrid control scheme. The effectiveness of proposed pitch control system and the CFD results are validated by building an H-type VAWT prototype. This prototype is tested outdoor extensively at different wind conditions for both fixed and variable pitch angle configurations. Results demonstrate that the blade pitching technique enhanced the performance of an H-type VAWT in terms of power output by around $22 \%$. 


\section{ACKNOWLEDGEMENTS}

In the name of Allah, the most beneficent and the most merciful. I wish to express my profound gratitude to Allah for his grace and mercy granted to me right from birth to date, "Thanks my God".

My profound gratitude goes to my country Libya for the support and grand even during the most difficult situations and unstable conditions.

I wish to render my unreserved gratitude to my project supervisors Prof. Fue-Sang Lien and Prof. William Melek for their support, guidance and time given to me during this work. You gave me all the support and freedom I would ever need to conduct experiments and do an original research toward this $\mathrm{PhD}$ thesis. I will never forget it, thank you and God bless you. I would also like to show my appreciation to Egyptian Internship students especially Eng. Mohamed A. Daoud for his help to finish the experimental control part. 


\section{DEDICATION}

This is dedicated to the one I love. My heartfelt gratitude goes to my mother Kideaga A. and to the spirit of my father Yousef Abdalrahman. Also, my wife's Ayea Omar for her support both morally and spiritually, and for her patience and love. I also would like to dedicate it to my brothers and sisters for their support and encouragement. 


\section{TABle OF CONTENTS}

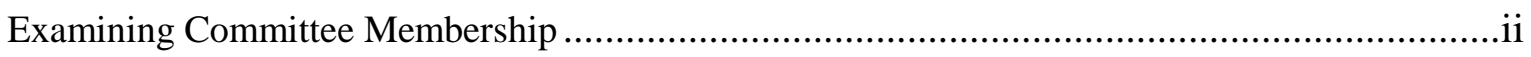

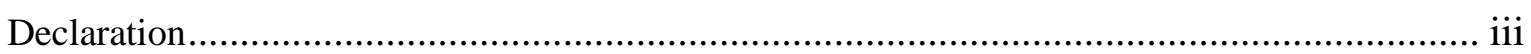

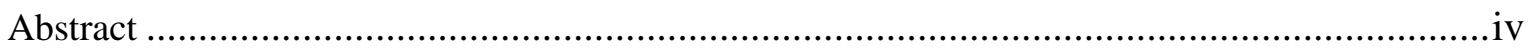

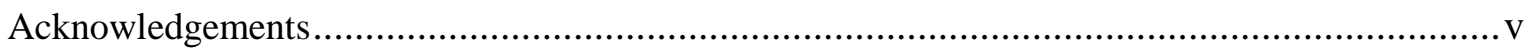

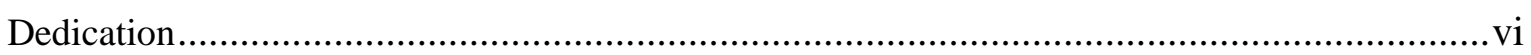

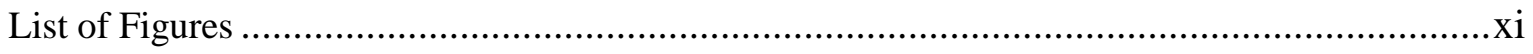

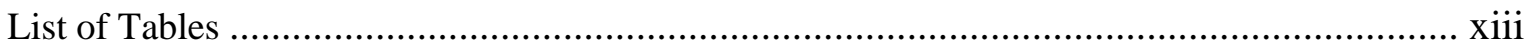

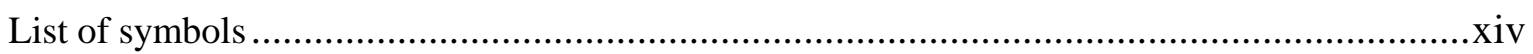

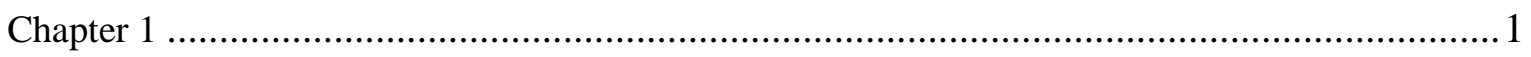

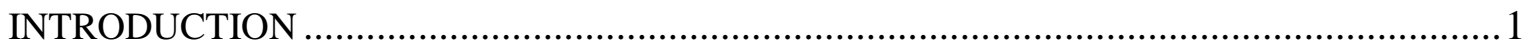

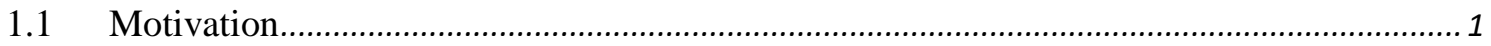

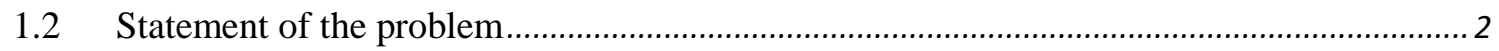

$1.3 \quad$ Proposed thesis contributions and novelty ........................................................................

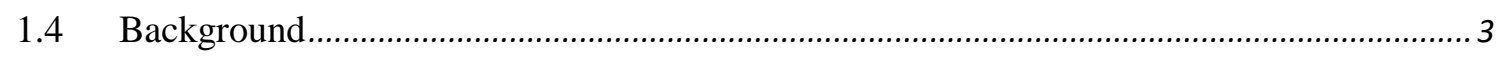

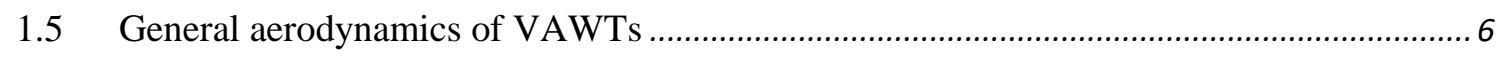

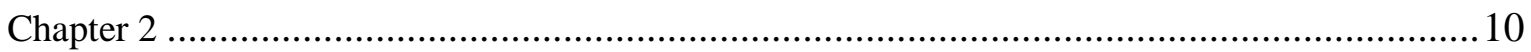

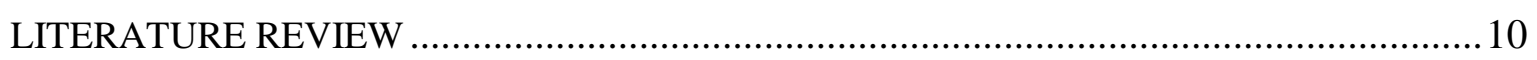

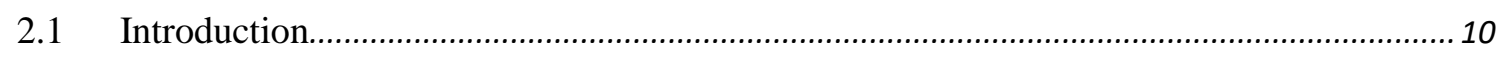

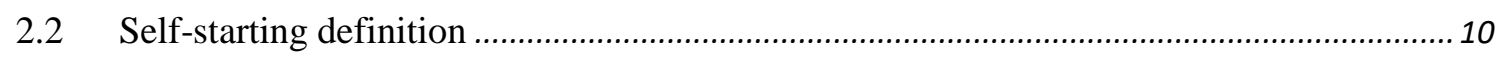

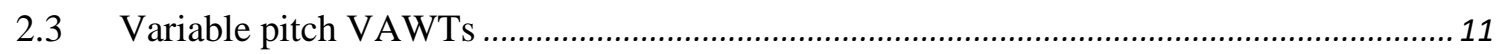

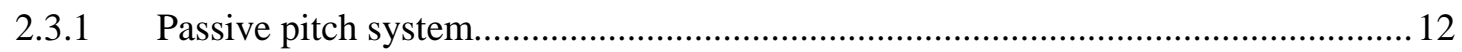

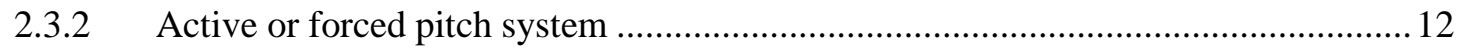

2.4 Analytical and numerical studies for investigating the aerodynamic of VAWTs ..............13

2.5 Studies of wind turbine pitch control system design .......................................................... 16

2.6 Methodology 
COMPUTATIONAL FLUID DYNAMICS (CFD) MODEL................................................. 20

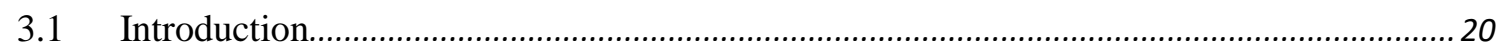

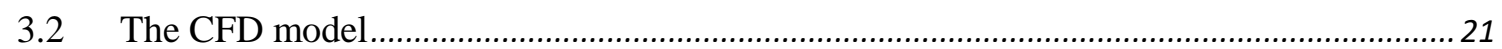

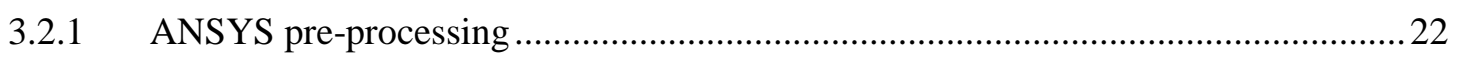

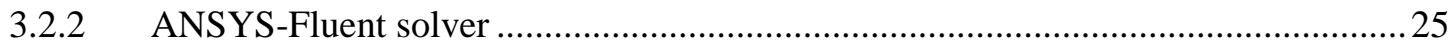

3.3 Validation

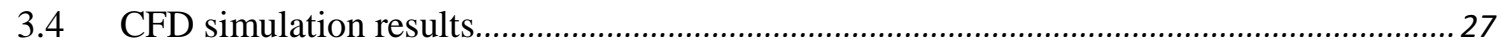

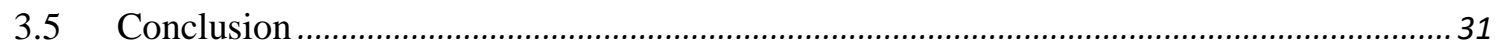

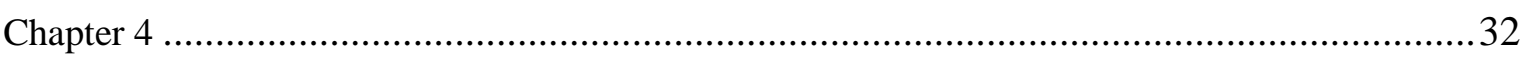

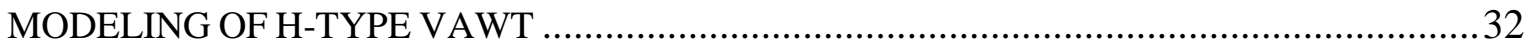

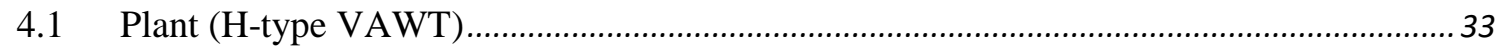

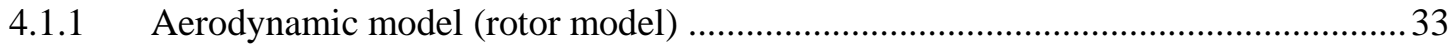

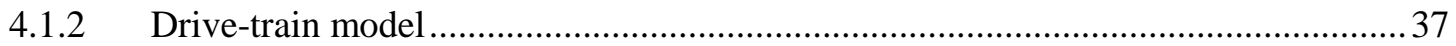

4.1.3 Model of Permanent Magnet Synchronous Generator (PMSG) ................................ 37

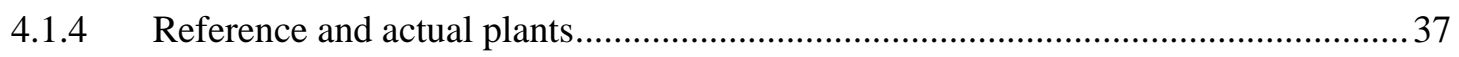

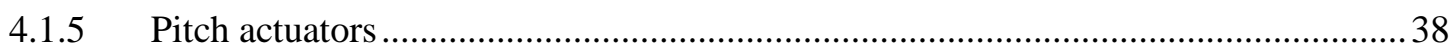

4.2 Proposed H-type VAWT blade pitch control system ……………….................................... 38

4.2.1 Using an MLP-ANN for mapping of a variable pitch H-type VAWT rotor.............. 40

4.2.2 Using an MLP-ANN for the blade pitch angle control system ..................................40

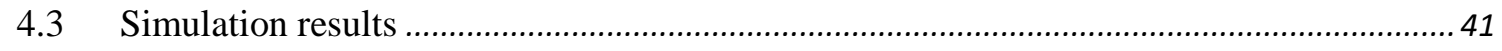

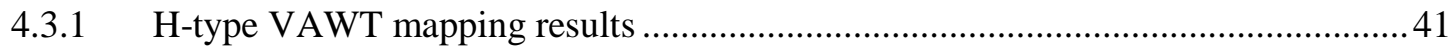

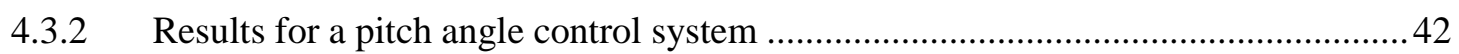

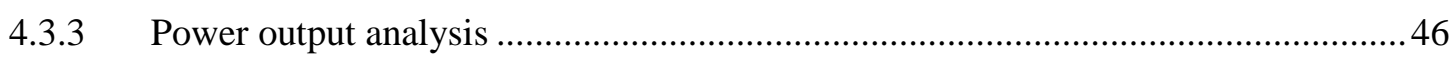

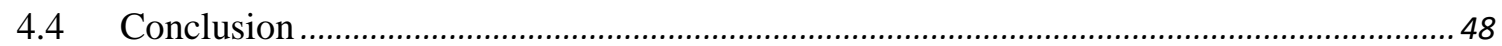

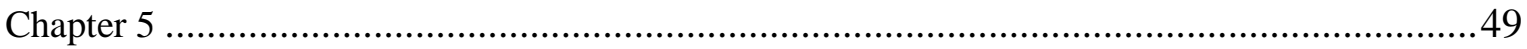

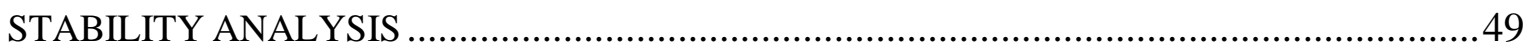

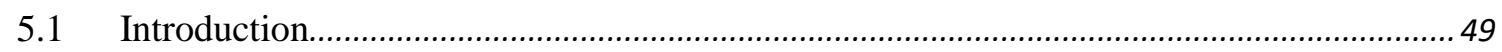

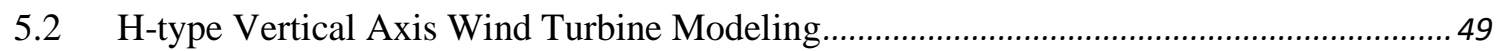

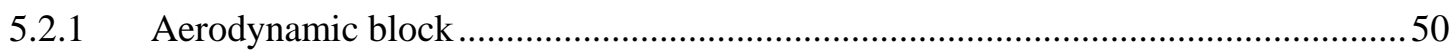




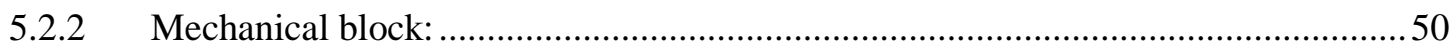

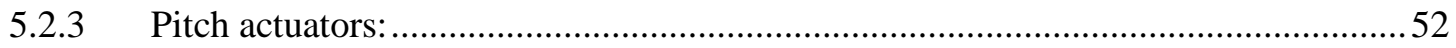

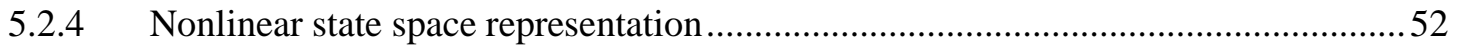

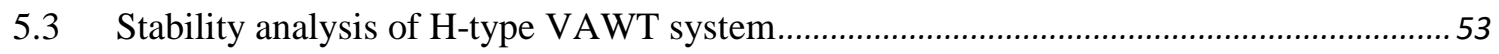

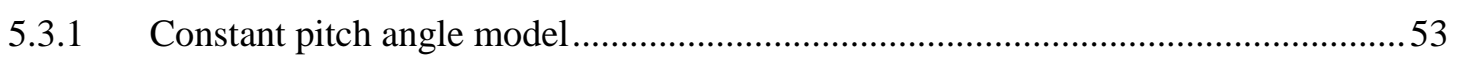

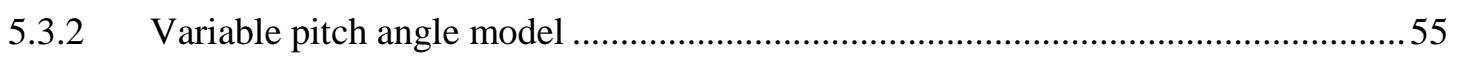

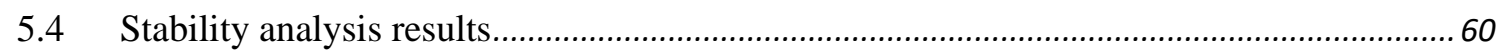

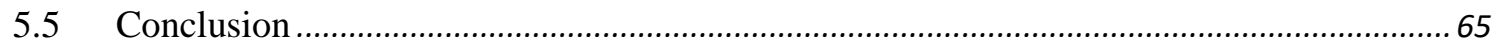

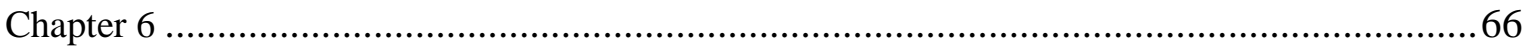

AN H-TYPE VAWT EXPERIMENTAL SETUP: DESIGN AND VALIDATION....................66

6.1 Design, Manufacturing \& Assembly an H-type VAWT .....................................................6

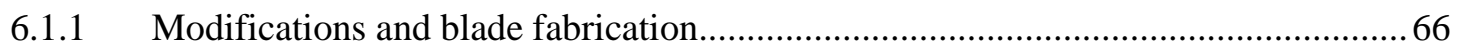

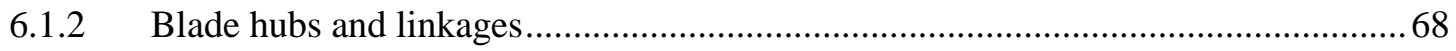

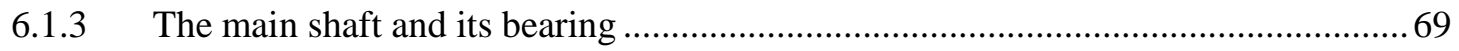

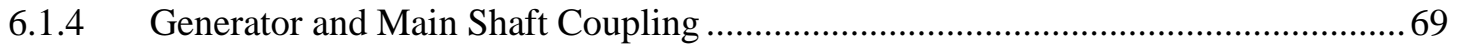

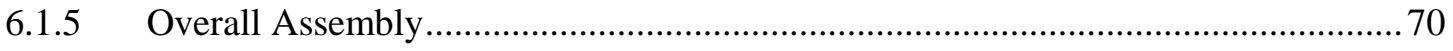

6.2 Design and implementation of blade pitch control system for an H-type VAWT .............. 72

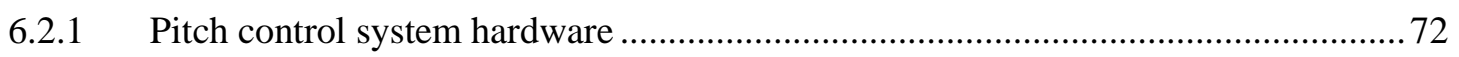

6.2.2 Data Acquisition system interface (DAQ system) .................................................. 74

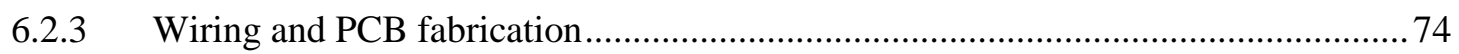

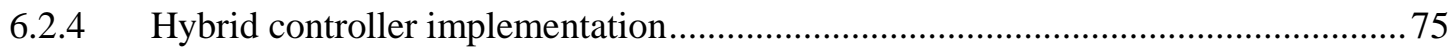

6.2.5 Feedback loop of the blade pitching system ............................................................. 77

6.2.6 Experimental measurements ..................................................................................

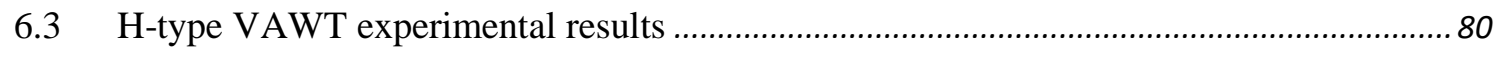

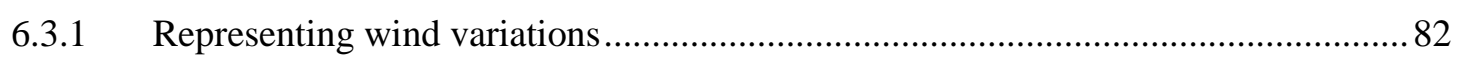

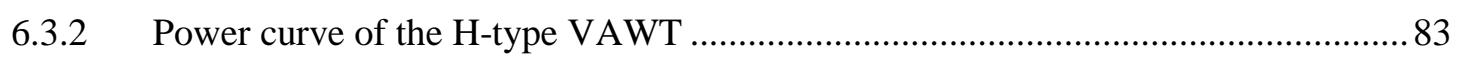

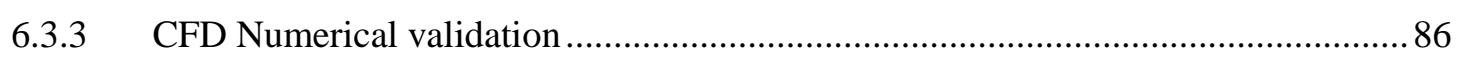

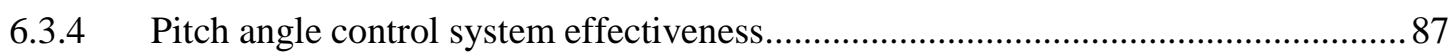

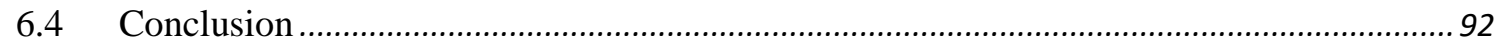

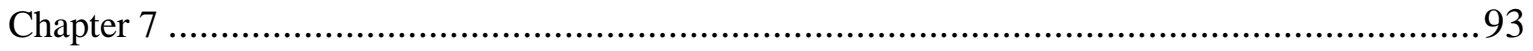

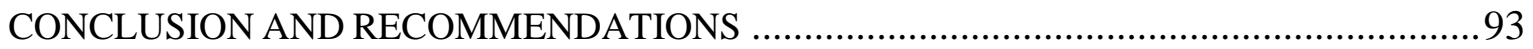




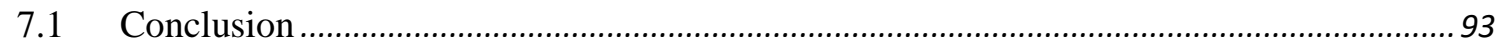

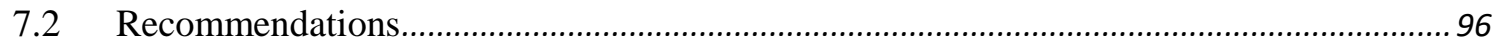

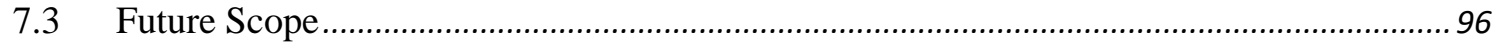

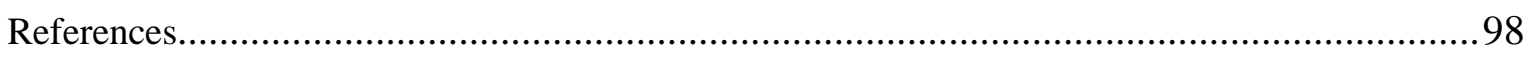

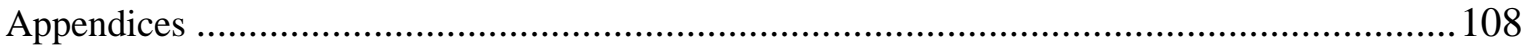




\section{LIST OF FIGURES}

Figure 2.1. The $C p$-TSR curves for Sandia $17 m$ VAWT and Mod-0A 27.8m HAWT [25]................ 11

Figure 3.1. Main computational subdomains used in ANSYS Design Modeler...................................... 23

Figure 3.2. Mesh of the Darrieus turbine modeled for this study. ....................................................23

Figure 3.3. Comparison of the CFD results for this study with other published experimental and CFD results, with respect to the power coefficient versus the TSR at different pitch angles.

Figure 3.4. Moment coefficient variations for the blade 1 at low TSR of $\lambda=1$ with different pitch angles. 29

Figure 3.5. Optimum pitch angles for three blades at different TSRs. ............................................. 30

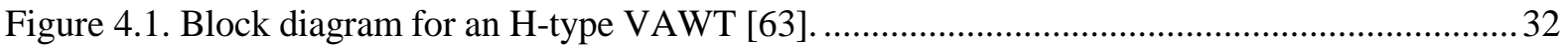

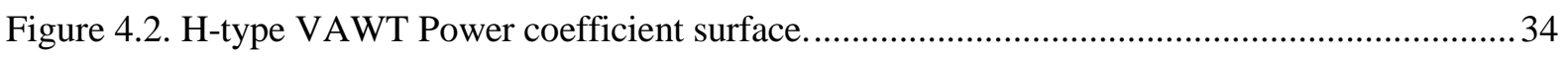

Figure 4.3. LUT-mapping of the power coefficient $\left(C_{p}\right)$ for a fixed pitch angle VAWT rotor............... 35

Figure 4.4. MLP-ANN mapping of the power coefficient $\left(C_{p}\right)$ for a variable pitch angle VAWT rotor.

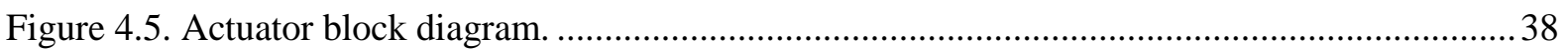

Figure 4.6. Proposed blade pitch control system block diagram......................................................... 39

Figure 4.7. Mapping of the power coefficient $\left(C_{p}\right)$ at a wind speed of $10 \mathrm{~m} / \mathrm{s}$ by (a) the LUT for a

fixed pitch angle VAWT and (b) the MLP-ANN for a variable pitch angle VAWT..................... 41

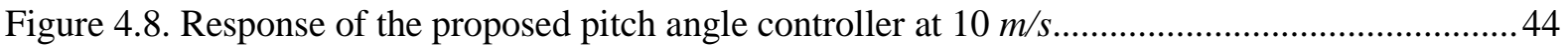

Figure 4.9. Power output from fixed and variable pitch angle H-type VAWT at uniform wind speed. 45

Figure 5.1. A wind turbine drive-train model based on a 2-mass model..............................................50

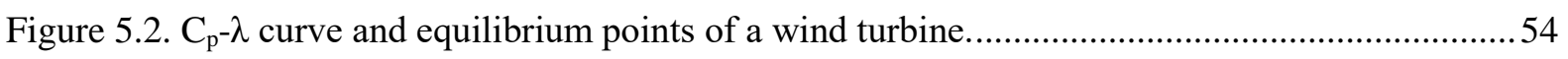

Figure 5.3. Equilibrium points for variable pitch angles wind turbine [87]......................................57

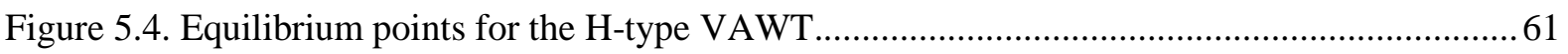

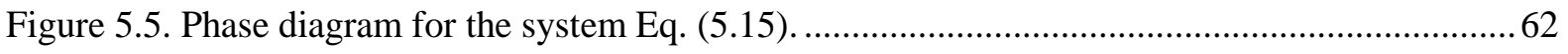

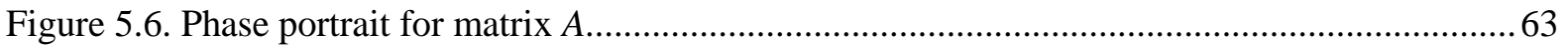

Figure 5.7. Stability domains for $C_{p}-\lambda$ curves at different pitch angles. ..............................................64

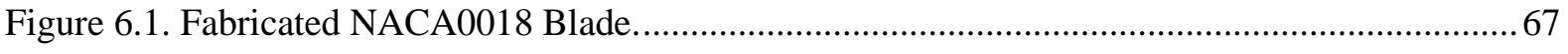

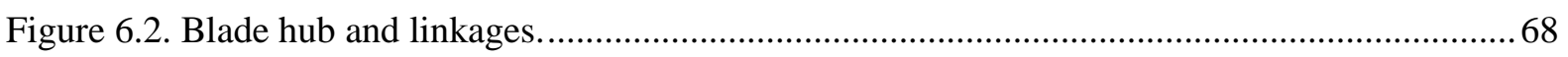

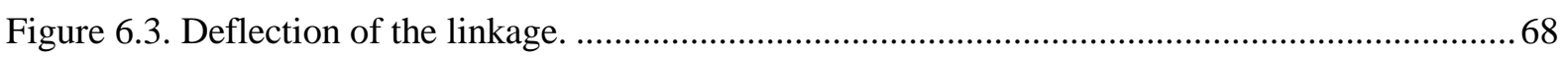

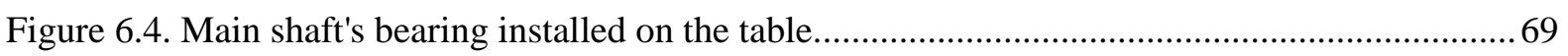

Figure 6.5. Pulley system for main shaft and generator coupling ........................................................ 70

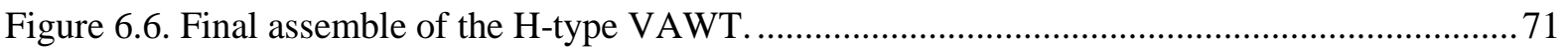

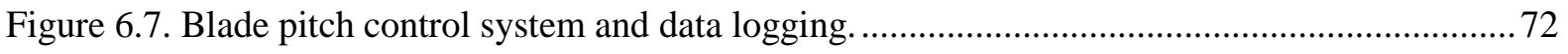

Figure 6.8. Four-bar linkage, DC motor, stopper, and blade encoder experimental setup. .................. 73

Figure 6.9. CAD design and main dimensions of four-bar linkage system. ....................................... 73 


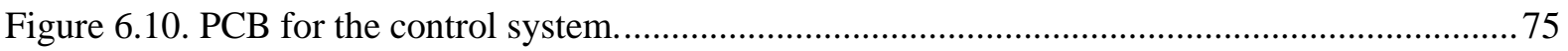

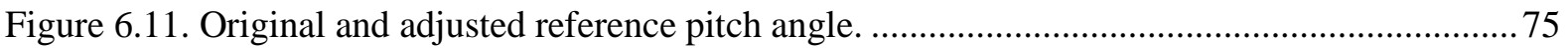

Figure 6.12. Matlab script for the MLP-ANN implemented in LabView............................................. 76

Figure 6.13. A block diagram of the closed-loop pitch control system for one blade. ......................... 77

Figure 6.14. Absolute rotary encoder experimental setup. …............................................................... 78

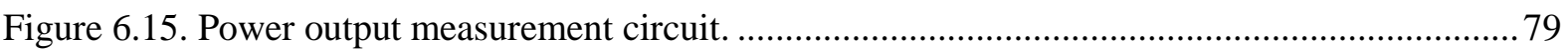

Figure 6.16. A pickup truck for H-type VAWT experimental setup. .................................................. 80

Figure 6.17. Main road for Test 1 and Test 2 and ground wind speed for Test 1 ............................... 81

Figure 6.18. Normal probability plot (P-plot) for wind speed data...................................................... 82

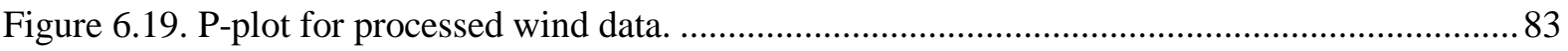

Figure 6.20. The H-type VAWT power generation of first and second tests in both fixed and variable

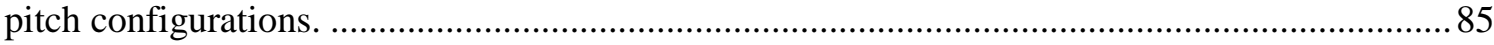

Figure 6.21. Filtered power generation curves for both tests in fixed and variable pitch angle

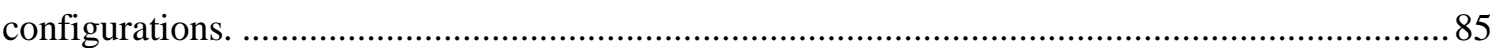

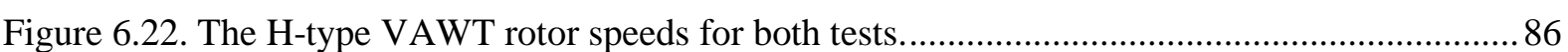

Figure 6.23. Power coefficient comparison between the experimental and CFD results. ..................... 87

Figure 6.24. The Fréchet distance of experimental and numerical (CFD) power coefficient curves. ..87 Figure 6.25. Response of the Hybrid controller for each blade at different RPMs for three revolutions. 


\section{LIST OF TABLES}

Table 3.1. Main features of the Darrieus wind turbine analyzed in the present study........................2

Table 3.2. Mesh features for current VAWT model. ........................................................................... 24

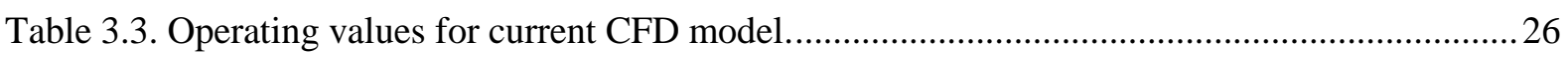

Table 3.4. Power coefficients $\left(C_{p}\right)$ at different TSRs and pitch angles.............................................2 28

Table 4.1. Rise time for both the MLP-ANN and Hybrid pitch control systems at a uniform wind

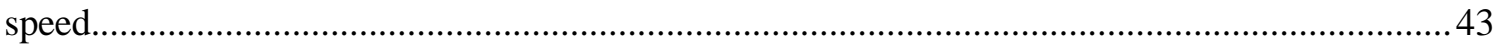

Table 4.2. MSEs for both the MLP-ANN and Hybrid pitch control systems at a uniform wind speed. 43

Table 4.3.Wind energy prediction using fixed and variable pitch angle H-type VAWT models. ......... 46

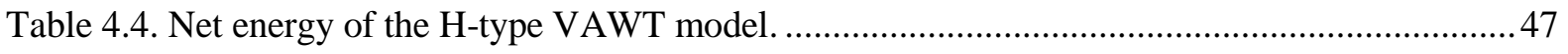

Table 5.1. Stability results using theorem i for the H-type VAWT model ..........................................61

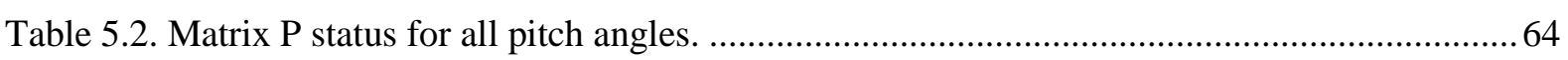

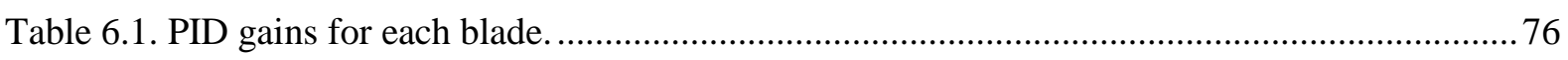

Table 6.2. RMSE of the Hybrid controller in different weights. ....................................................... 90

Table 6.3. Overshoot of the Hybrid controller response for the first revolution..................................91 


\section{LIST OF SYMBOLS}

\begin{tabular}{|c|c|}
\hline$\alpha$ & Angle of attack \\
\hline$\beta$ & Blade pitch angle \\
\hline$\theta$ & Rotor position angle (Azimuth angle) \\
\hline$\lambda$ & Tip speed ratio \\
\hline$\omega_{r}$ & Rotor angular velocity \\
\hline$u_{\infty}$ & Uniform wind speed \\
\hline$\sigma$ & Solidity \\
\hline$\rho$ & Air density \\
\hline$\tau_{t}$ & Rotor torque \\
\hline$\varphi$ & Angle of relative wind \\
\hline$\Delta t$ & CFD time step size \\
\hline$\lambda_{o p t}$ & Optimum tip speed ratio \\
\hline$\mu$ & Mean of wind speed data \\
\hline$R$ & Rotor radius \\
\hline$N_{b}$ & Number of blades \\
\hline$c$ & Blade chord \\
\hline$D$ & Rotor diameter \\
\hline$\vec{V}$ & Tangential velocity vector \\
\hline $\overrightarrow{\mathrm{W}}$ & Relative velocity vector \\
\hline $\overrightarrow{\mathrm{U}}$ & Induced velocity vector \\
\hline$C_{L}$ & Lift coefficient \\
\hline$C_{D}$ & Drag coefficient \\
\hline$C_{m}$ & Moment coefficient \\
\hline$A$ & Swept area \\
\hline $\mathrm{H}$ & Blade height \\
\hline $\mathrm{T}$ & Tangential force \\
\hline$N$ & Normal force \\
\hline$P_{m}$ & Mechanical power \\
\hline$C_{p}$ & Power coefficient \\
\hline$P_{W}$ & Power in the wind \\
\hline$C_{m, \max }$ & Max. Moment coefficient \\
\hline$\beta_{\text {opt }}$ & Optimum pitch angle \\
\hline$y+$ & Wall distance \\
\hline I & Turbulent intensity \\
\hline$l$ & Turbulent length scale \\
\hline $\bar{C}_{m}$ & Average moment coefficient \\
\hline $\bar{C}_{m, \text { total }}$ & Average moment coefficient (all blades) \\
\hline$e$ & Error \\
\hline$C_{p, a v g}$ & Average power coefficient \\
\hline$P_{r e f}$ & Reference power output \\
\hline$P_{g}$ & Ele. Power generation \\
\hline$T_{r}$ & Control rise time \\
\hline$E_{a}$ & Energy by pitching \\
\hline Ev & Gross energy for variable pitch angle case \\
\hline$E f$ & Gross energy for fixed pitch angle case \\
\hline$E_{\text {net }}$ & Net energy \\
\hline$E_{\text {loss }}$ & Energy losses \\
\hline$P_{\text {loss }}$ & Power losses \\
\hline$P_{\text {servo }}$ & Power losses by servomotor \\
\hline$P_{C}$ & Power losses by centrifugal force \\
\hline$T_{\mathrm{s}}$ & Servomotor torque \\
\hline
\end{tabular}




$\begin{array}{cl}I & \text { Moment of inertia for blade } \\ m_{\text {blade }} & \text { Mass blade } \\ D_{r} & \text { Rotor self-damping } \\ D_{g} & \text { Generator self-damping } \\ k & \text { Turbine gear ratio } \\ J_{r} & \text { Moments of inertia of the rotor } \\ J_{g} & \text { Moments of inertia of the generator } \\ J_{t o t} & \text { Total moments of inertia of the drive system } \\ C_{p, e q} & \text { Equilibrium point of power coefficient } \\ V_{r m s} & \text { Root mean square voltage } \\ Z & \text { Z-scores }\end{array}$




\section{Chapter 1}

\section{INTRODUCTION}

\subsection{Motivation}

Because of the rising worldwide demand for energy, the development of alternative and renewable energy sources has become a vital factor in providing clean energy. All forms of renewable energy, from biofuels and hydro to wind, geothermal energy, and solar power have been explored in terms of the practical and economic potential of the renewable energy sources. The horizontal axis wind turbines (HAWTs) industry has received considerable attention compared to that of vertical axis wind turbines (VAWTs) because of their high efficiency. Most VAWTs operate at a relatively low tip speed ratio (TSR) of only 2 to 3 [1]. Yet, they can achieve some useful power production with less noise generation. In recent years, there has been a noticeable interest in developing the small and medium-sized range of VAWTs because they can be used in both rural electrification as stand-alone power supply systems as well as in urban environments. The limited research available about VAWTs clearly indicates that the aerodynamic characteristics of the Darrieus VAWT can be improved using some strategies such as blade pitching. However, the design of the active pitch control system for a multi-bladed VAWT is a challenging problem due to the difficulty in defining optimum pitch angles during a given revolution in the presence of uncertainties due to varying wind speed, direction and wake effects around the blades. Therefore, intelligent control methods which utilize an neural networks approach are proposed in this thesis to design an individual blade active pitch control system for an H-type VAWT to enhance its performance in terms of power generation and subsequently increase its adoption as a standalone wind energy generation source. In this thesis, an H-type VAWT is simulated using a CFD model and validated experimentally along with the proposed control method by building a full scale model of an H-Darrieus VAWT. 


\subsection{Statement of the problem}

During the rotation of an VAWT, the flow velocity around the blades changes constantly in both the upstream and downstream regions. At low TSRs, the poor self-starting capability occurs due to the negative torque. Particularly, the negative torque is often generated by fixed pitch VAWTs because of the large dynamic cyclic variations of angle of attack ( $\alpha)$ [2]. How to control of the variation of the angle of attack plays an important role in improving the power performance of VAWTs. Blade pitching technique is proposed herein to avoid or reduce the effect of dynamic stall due to the large variation of angle of attack, and hence, improve the VAWT performance in terms of power output and self-starting capability.

\subsection{Proposed thesis contributions and novelty}

The small and medium size of VAWTs can be effectively utilized as stand-alone wind energy generation sources if its state of the art performance can be improved in terms of efficiency by incorporating an intelligent controller. To this end, the variable pitch system is proposed to enhance the VAWT efficiency. Recent CFD studies [3][4][5][6] have examined effect of blade pitch angles on power output of VAWTs, and the blade pitch angles were chosen randomly or calculated by using a sinusoidal pitch function (i.e., $\beta(\theta)$ ), to only numerically investigate their effects on VAWT performance and/or self-starting capability. These pitch angles were also used in some experimental studies [7][8][9] as "optimal pitch angles". These pitch angles are, however, not usually the optimal values because they are not guaranteed to generate the maximum moment coefficients, and subsequently, the maximum power coefficients for blades at all azimuthal angle position $(\theta)$. Also, the experimental studies used mechanical designs such as cams or simple linear pitch control systems to set the blades at the desired pitch angles.

Simulation, modeling, and fabrication of an H-type VAWT are carried out in this thesis. The goal is to systematically design a nonlinear individual active blade pitch control system 
for an H-type VAWT based on the simulation results obtained with the commercial ANSYSFluent CFD software. The CFD model was used for studying the aerodynamic performance of an H-type VAWT, including its self-starting capability, and determining the optimum pitch angles for each individual blade. These optimal pitch angles are also utilized in the design of the proposed blade pitch control system. If each blade can be controlled individually, it is possible to maximize the moment coefficients for all blades. Because of the complexity of VAWT aerodynamics, a reliable nonlinear control system is required. Therefore, this thesis proposes the use of intelligent control techniques based on the neural network approach combined with PID feedback control mechanism (i.e., Hybrid controller) to design an individual active blade pitch control system for an H-type VAWT as a means of improving its power generation performance. The nonlinear state space model of an H-type VAWT is also derived as part of the stability analysis for the proposed pitch control system using Lyapunov stability theory. In addition, several outdoor experiments are conducted in order to investigate the effect of the blade pitching technique on the performance of an H-type VAWT and validate the effectiveness of the proposed control system.

\subsection{Background}

According to the International Energy Agency's statistics, about 1.3 billion people, most of whom are living in remote areas or islands, have no access to electricity [10]. All forms of renewable energy, from biofuels and hydro to wind, geothermal energy, and solar power, have been explored in terms of their practical and economic potential. Stand-alone power supply systems, such as wind turbines and solar cells, are not only suitable solutions for producing the electricity needed for rural and remote areas, but also economical alternatives because they can contribute to a reduction in the cost of grid extensions [11]. Because standalone power systems do not rely on the utility grid, they can utilize renewable energy technologies such as wind turbines [10]. Wind energy is one of the most promising renewable 
energy. For a number of years, two main types of wind turbines have been used for the extraction of power from wind. They are categorized on the basis of their orientation of the axis of rotation: vertical axis wind turbines (VAWTs) and horizontal axis wind turbines (HAWTs).

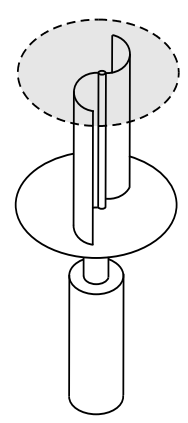

(a)

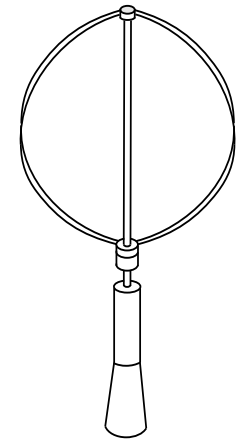

( b )

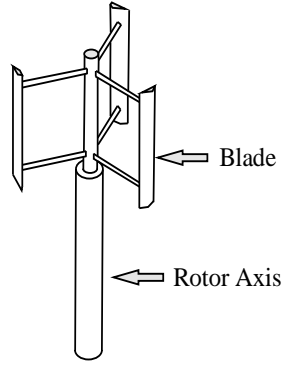

(c)

Figure 1.1. Some types of VAWTs; (a): Savonius- Rotor (drag type), (b): Darrieus- Rotor (lift type), (c): H-Darrieus- Rotor [12].

Vertical Axis Wind Turbines (VAWTs) fall into two categories of lift-based or drag-based [12]. Savonius is a drag-type device with two or more half cylinder shape blades as shown in Fig. 1.1 (a), while Darrieus, which was developed by Georges Jean Marie Darrieus in 1925, is a lift type of VAWT. Specifically, there are different kinds of Darrieus rotors. For instance, the "egg-beater" or "troposkein" Darrieus shape with curved blades is shown in Fig. 1.1 (b). Also, there is the "H-rotor" or "Giromill" in which the blades are straight and parallel to the axis of rotation as shown in Fig. 1.1 (c). The airfoil profile of blades can create aerodynamic lift when they are exposed to the incident wind. This aerodynamic lift produces a moment about the blade axis that allows the main shaft of wind turbine to rotate. The straight-bladed Darrieus VAWTs are more popular for the small-scale power production because of blade design simplicity. In addition, the straight-bladed Darrieus VAWTs can use a variable pitch angle. The variable pitch configuration for blades is proposed to improve the starting torque issues of Darrieus VAWTs. Moreover, VAWTs offer a number of advantages over HAWTs. For example, VAWTs can receive the wind blowing from any direction (i.e., omni-directional), so 
that a yaw mechanism is not needed. In addition, maintenance is relatively quick and easy since the transmission equipment and generator are placed at the ground level. Also, because of simplicity of blade design, the cost is relatively low [13]. For these reasons the work presented in this thesis focuses on the performance of the Darrieus H-type Vertical-Axis Turbine (H-type VAWT). Some of the most important differences between Darrieus vertical and horizontal axis wind turbines are summarized in Table 1.1 [14].

Table 1.1 Main differences between vertical and horizontal axis wind turbines [14].

\begin{tabular}{llll}
\hline & $\begin{array}{c}\text { H-type } \\
\text { Darrieus } \\
\text { VAWT }\end{array}$ & $\begin{array}{c}\text { Curved-blade } \\
\text { Darrieus VAWT }\end{array}$ & HAWT \\
\hline Blade Profile & Simple & Complicated & Complicated \\
Need for Yaw Mechanism & No & No & Yes \\
Possibility of Pitch Mechanism & Yes & No & Yes \\
Tower & Yes & No & Yes \\
Guy Wires & Optional & Yes & No \\
Noise & Low & Moderate & High \\
Blade Area & Moderate & Large & Small \\
Generator position & On ground & On ground & On tower \\
Self-start & No & No & Yes \\
Tower interference & Small & Small & Large \\
Foundation & Moderate & Simple & Extensive \\
Overall Structure & Simple & Simple & Complicated \\
Blade load & Moderate & Low & High \\
\hline
\end{tabular}




\subsection{General aerodynamics of VAWTs}

An important parameter associated with VAWTs is the operating tip speed ratio $\operatorname{TSR}(\lambda)$, and a key factor in the selection of TSR is wind speed, as given in [15].

$$
\lambda=\frac{\omega_{r} R}{u_{\infty}}
$$

where $\omega_{r}$ is the angular velocity in $(\mathrm{rad} / \mathrm{sec}), u_{\infty}$ is the wind speed in $(\mathrm{m} / \mathrm{s})$ and $R$ is the rotor radius in $(m)$. The second dimensionless parameter is solidity $(\sigma)$ which is defined as the ratio of rotor blade surface area to the frontal, swept area of the wind turbine covered by the blades and is given by the following expression [16]

$$
\sigma=\frac{N_{b} c}{D}
$$

Here, $N_{b}$ is the number of blades, $c$ is the blade chord length in $(m)$, and $D$ is the diameter of the rotor in $(m)$. The geometry of the VAWT can be defined using solidity. Once the tip speed ratio and geometry are defined, Actual VAWT performance can be predicted based on the aerodynamic force acting on each blade. Figure 1.2 illustrates the velocity and force vectors acting on a Darrieus turbine blade. The velocity $(\vec{V})$ is the tangential velocity of the rotor $\left(-\overrightarrow{\omega_{r}} \times \vec{R}\right)$. The resultant velocity vector $(\overrightarrow{\mathrm{W}})$ is the relative velocity vector that consists of the induced velocity $(\overrightarrow{\mathrm{U}})$ and $(\overrightarrow{\mathrm{V}})$. The angle of attack $(\alpha)$ is typically defined as the angle between the direction of the relative velocity, W, and the chord line of the blade. Obviously, both the angle of attack $(\alpha)$ and the relative wind speed (W), which is a function of the azimuth angle $(\theta)$, vary during each cycle. The result is that the magnitude and orientation of both the lift and drag forces change depending on the azimuthal position of the blade. The induced velocity $(\overrightarrow{\mathrm{U}})$ is lower than the freestream velocity $\left(u_{\infty}\right)$ due to the pressure drop across the rotor. 


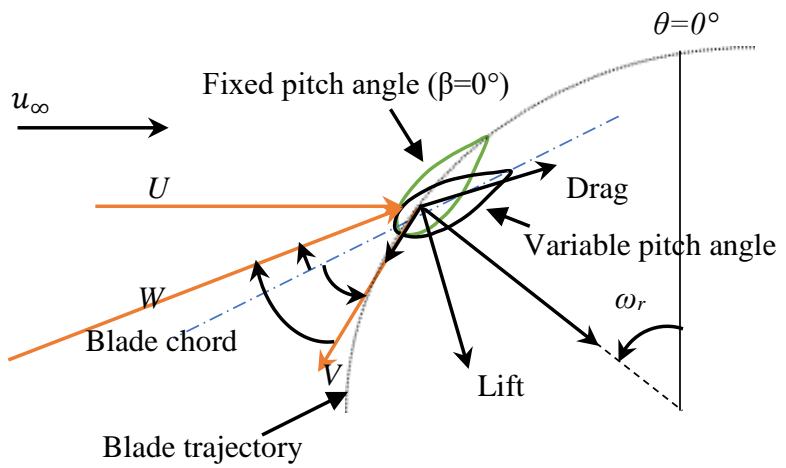

Figure 1.2. Forces and velocities acting on a Darrieus turbine blade for various azimuthal positions [17].

The variation of angle of incidence is associated with the dynamic stall phenomenon that occurs at a relatively large angle of attack greater than the static stall angle. The blade angle of attack $(\alpha)$ for a VAWT can be expressed as [18]:

$$
\alpha=\tan ^{-1}\left(\frac{\cos \theta}{\sin \theta+\lambda}\right)
$$

The blades of the VAWT frequently experience a high angle of attack beyond the stall angle at low TSRs as shown in Fig. 1.3. This means that the blades stall during most of their trajectories [19]. Consequently, the stall leads to a sudden decrease in the lift, a rapid increase in the drag and hence a decrease in the rotor torque. Figure 1.4 explains the lift and drag coefficients $\left(C_{L}\right.$ and $\left.C_{D}\right)$ and the resultant velocity $(\overrightarrow{\mathrm{W}})$ variations for a fixed pitch VAWT at a low TSR. It is clear that the drag is dominant for most of the periodic blade trajectory [20].

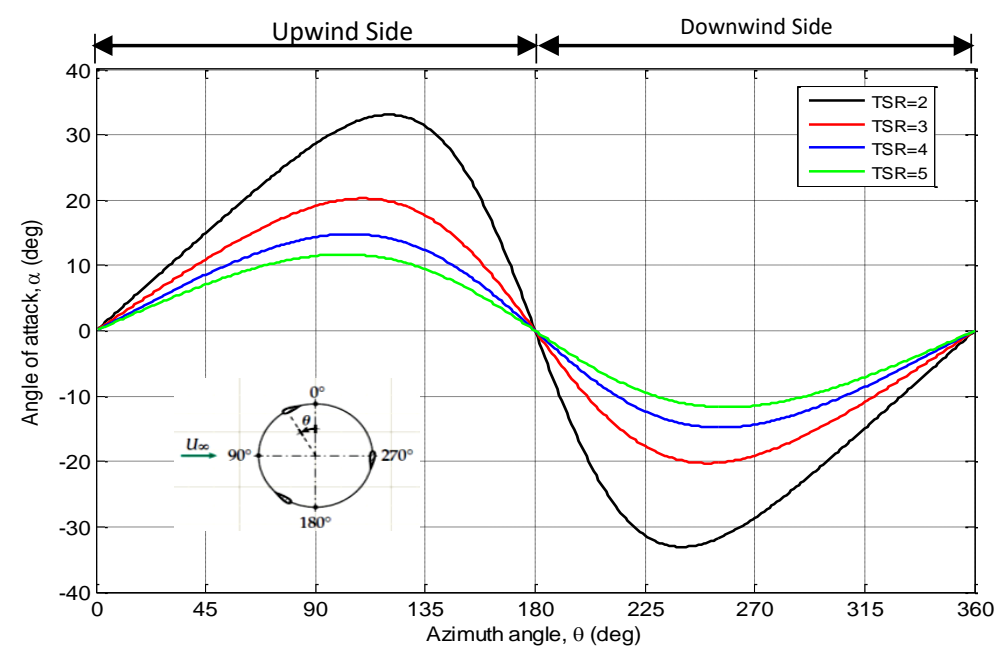

Figure 1.3. Variation of angle of attack $(\alpha)$ at different tip speed ratios $(\lambda)$ [19]. 


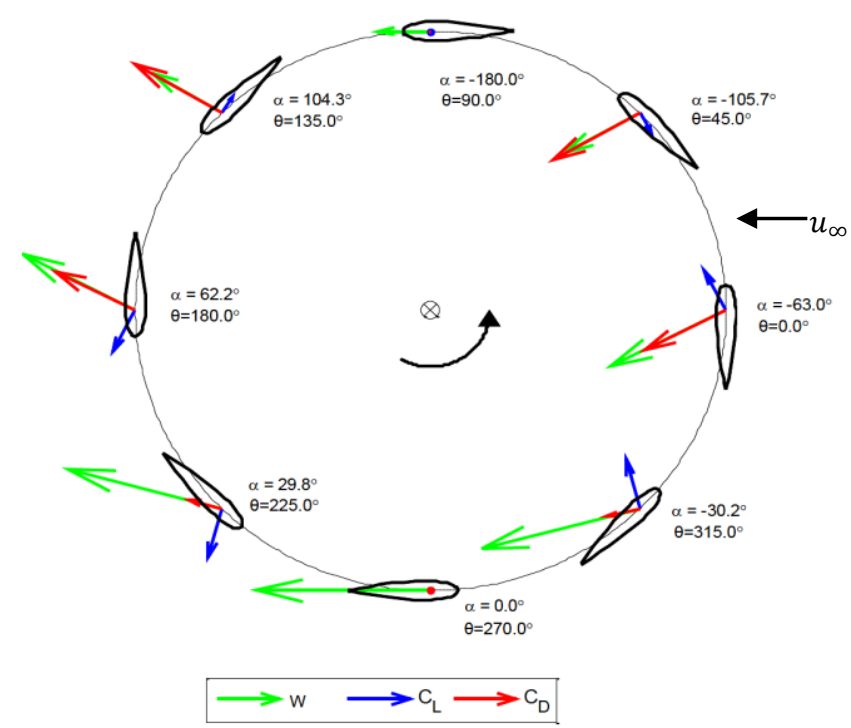

Figure 1.4. Variations of aerodynamic forces (lift and drag) and the relative velocity (W) at different azimuthal angels around a Darrieus rotor [20].

The large variation of angle of attack can also increase the development of a blade vortex which drives to a sudden flow separation on the suction surface of the blade. Figure 1.5 shows that the vortex travels from the blade leading edge to the trailing edge as it grows (1a to $1 b)$, and then separates from the airfoil surface close to the trailing edge ( $2 a$ and $2 b)$. It produces the vortex shedding in the upstream blade (2 and 3) which moves to the downstream blades (4). Consequently, the flow field around a VAWT involves a complex vortex structure [21].

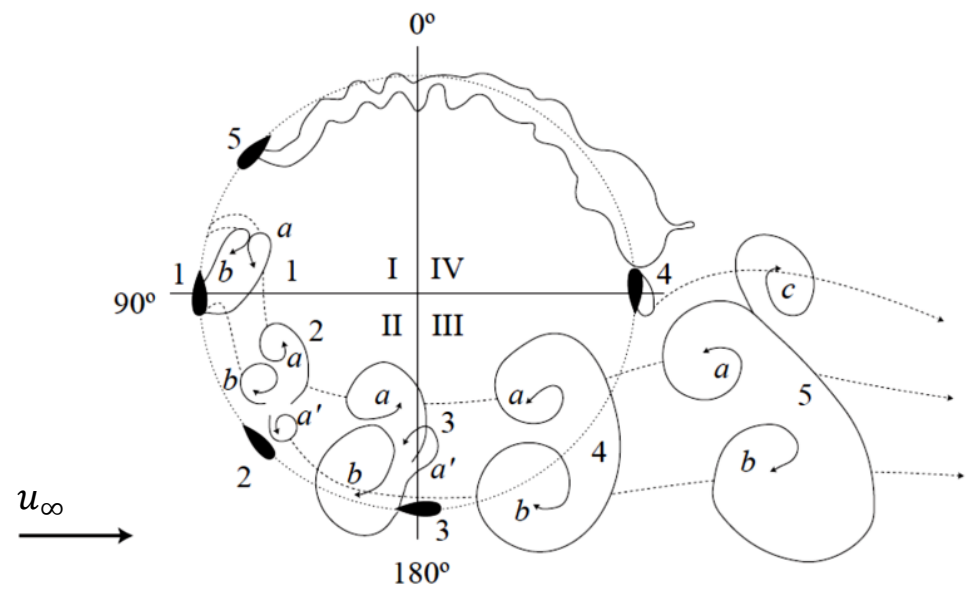

Figure 1.5. A schematic picture of the vortex shedding phenomenon during the operation of an H-type VAWT at a low TSR ( $a, a^{\prime}, b$ and $c$ denote vortices) [22]. 
In order to study the aerodynamic characteristics around the blade such as dynamic stall, boundary layer etc., some coefficients should be taken into account such as lift, drag, and moment coefficients. These coefficients $\left(C_{L}, C_{D}\right.$, and $\left.C_{m}\right)$ can be expressed based on the relative wind speed, respectively, by [17]

$$
C_{L}=\frac{\text { Lift }}{1 / 2 \rho A W^{2}} ; C_{D}=\frac{\text { Drag }}{1 / 2 \rho A W^{2}} ; C_{m}=\frac{\tau_{t}}{1 / 2 \rho A u_{\infty}^{2} R}
$$

where $\rho$ is air density in $\left(\mathrm{kg} / \mathrm{m}^{3}\right), A$ is the swept area by the turbine in $\left(\mathrm{m}^{2}\right)$ (e.g., for an $\mathrm{H}$ type VAWT, $A=2 \mathrm{RH}$, where $\mathrm{H}$ is the blade length), and $\tau_{t}$ is the rotor torque in $(\mathrm{Nm})$. Figure 1.2 also shows the main forces acting on a VAWT blade, where the tangential (T) and normal (N) directions relative the blade velocity $\mathrm{V}$ are used to analyze the loads on the blades of a VAWT [23]. The amount of mechanical power, $P_{m}$, that can be absorbed by a wind turbine is:

$$
P_{m}=\omega_{r} \tau_{t}
$$

where $\omega_{r}$ is the rotational speed of rotor in $(\mathrm{rad} / \mathrm{sec})$. The rotor performance of VAWT can be estimated using the power coefficient $\left(C_{p}\right)$. It is the ratio of the mechanical power produced by the wind turbine $\left(P_{m}\right)$ to the power available in the wind $\left(P_{W}\right)$ [24]:

$$
C_{p}=\frac{P_{m}}{P_{W}}=\frac{\omega_{r} \tau_{t}}{1 / 2 \rho A u_{\infty}^{3}}=\frac{\omega_{r}\left(C_{m} \times 1 / 2 \rho A u_{\infty}^{2} R\right)}{1 / 2 \rho A u_{\infty}^{3}}=C_{m} \frac{\omega_{r} R}{u_{\infty}}=C_{m} \lambda
$$




\section{CHAPTER 2}

\section{LITERATURE REVIEW}

\subsection{Introduction}

Many methods have been proposed to improve the performance of VAWTs in terms of power generation and self-starting capability. The blade pitching is one of these methods that will be covered in this review.

\subsection{Self-starting definition}

Under no-load condition and stationary blades, the Darrieus VAWT can generate a small amount of forward torque and hence can start to rotate slowly. Because of negative torque, the extracted power per cycle is less than zero typically at tip speed ratios between 0.5 and 2.0 depending on the blade airfoil [25]. A large negative torque peak is the result of the blade operating within stall and post-stall conditions during its rotation, so the Darrieus VAWT cannot accelerate up to generate any power output. As a result, an external power such as a motor is required to accelerate rotor up, and hence, generate power [26]. Hill et al. [27] opined that there is no exact definition for the term self-starting. Many studies, however, suggest some criteria to define the term of self-starting as, for example, the ability of the rotor to accelerate from rest to its nominal operating speed without external power [28].

A good understanding of the VAWTs' complex aerodynamics is required to improve their performance in terms of power output generation. Because it has not been proven that the aerodynamic of HAWTs are fundamentally more efficient than VAWTs, It has been argued that VAWTs may be more appropriate than HAWTs on a very large scale (10 MW) due to the excessively increasing gravitational load on the HAWT blade [13]. Figure 2.1 shows that the measured peak power coefficient of the $17 \mathrm{~m}$ diameter Sandia Darrieus VAWT is generally comparable with the $37.8 m$ diameter Mod-0A HAWT [25]. Therefore, many studies are being conducted in order to enhance the self-starting capability and performance 
of VAWTs using different techniques [29]. For example, there are three suggested techniques for this purpose [25]:

- Variable pitch blades

- Flexible blades or sails

- Cambered, fixed pitch blades

The variable pitch mechanism is one of the most promising technologies to maximize the power of an H-Darrieus rotor type and improve its aerodynamic performance [7].

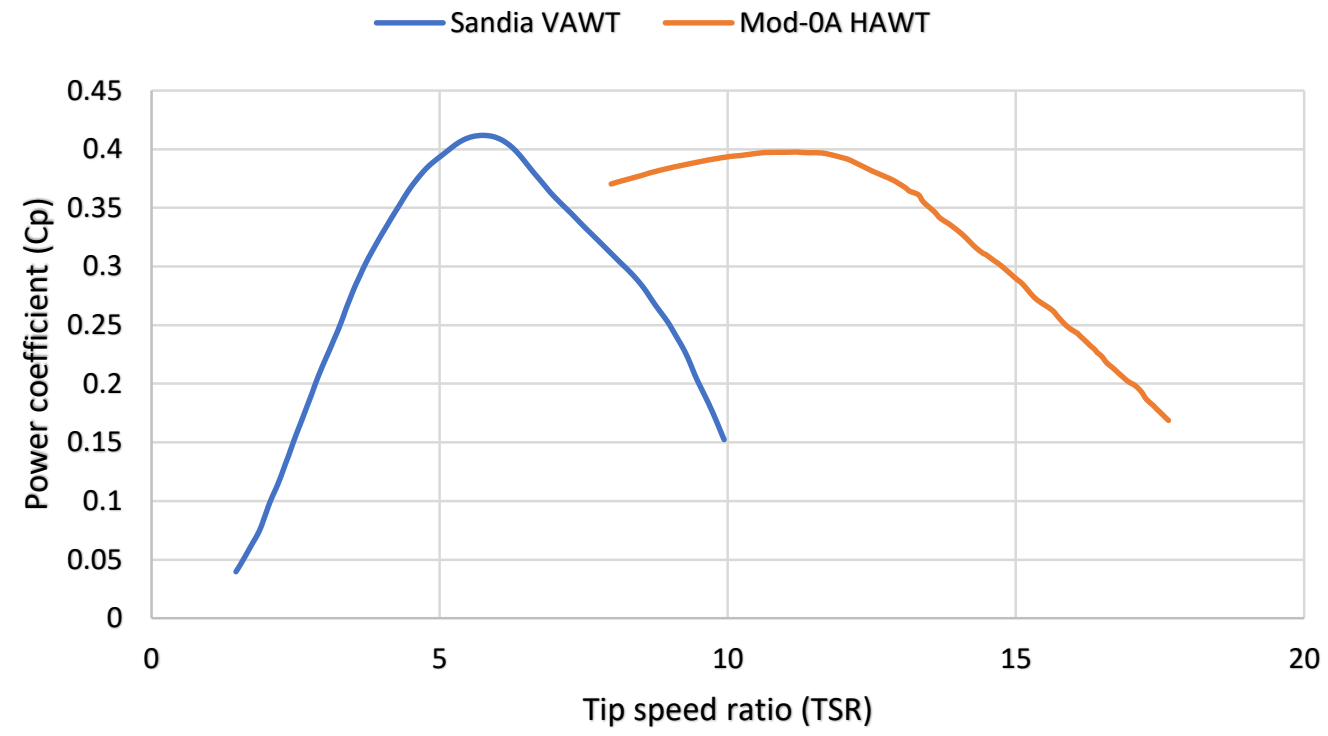

Figure 2.1. The $C p$-TSR curves for Sandia $17 m$ VAWT and Mod-0A 27.8m HAWT [25].

\subsection{Variable pitch VAWTs}

To improve VAWT performance, a variable pitch technique is applied through the modification of the angle of attack $(\alpha)$. Blade angles are shown in Fig. 1.2, where the blade pitch angle is set out as in [30]:

$$
\beta=\alpha-\varphi
$$

where $\varphi$ is the angle of relative wind. A variable pitch angle control system can be either of two main categories: passive or active [31]. 


\subsubsection{Passive pitch system}

In a passive variable pitch system, the blade is free to pitch about its axis near the leading edge. All passive variable pitch VAWTs depend on aerodynamic forces that produce a pitching moment about the pivot to reduce the angle of attack [32]. Some stabilizer configurations can be used including springs, counterweights or cable systems to limit the pitch angle. Although the angle of attack is reduced naturally using a passive pitch system, some disadvantages can be observed. Using the stabilizer configurations can generate extra forces such as spring forces. The impact of these forces can be obvious at a low wind speed.

\subsubsection{Active or forced pitch system}

In an active control system, pitch control mechanisms such as pushrods, cams, or servomotors have been designed to achieve continuous changes in the blade pitch amplitude. At low TSRs, a large pitch amplitude is needed in order to reduce the angle of attack, and hence, to enhance rotor performance. However, a large pitch amplitude can cause VAWT performance to deteriorate at high TSRs that it may be because a large blade pitching reduces the frontal area of an H-type VAWT to the extent that not enough power is produced. Conversely, a small pitch amplitude is sufficient to produce good performance at high TSRs, it will lead to poor performance at low TSRs which may be because a small pitch angle means that the effect of angle of attack still exists, resulting the blades are stalled for some of the time [24][26][33]. This effect gives rise to an important question, how should the pitch amplitude be varied in order to optimize VAWT performance as the TSR changes? In other words, how can optimum pitch angles be achieved during rotation to produce a maximum torque in a realistic condition? In fact, the proposed active pitch angle mechanisms by some studies [34]-[36] used simple techniques such as sinusoidal forcing through cam, gears, or servomotors. Moreover, the design of actuators with fast response times in order to vary the pitch angle for a small H-type VAWT is complicated due to the high frequency of the 
rotational speeds and the frequent changes in the angle of attack. For large VAWTs, such designs are also expensive. A suitable aerodynamic model and a control system that can approximate the optimal pitch angle of an H-type VAWT during rotation are therefore required. It can be argued that if self-starting of vertical axis wind turbines can be improved while retaining the simplicity of this model, such wind turbines can play an important role in providing power to the public without the need to access to grid power [32].

In this thesis, a $1.7 \mathrm{~m}$ diameter three-bladed H-type VAWT with a variable pitch angle and NACA 0018 airfoil are analyzed to efficiently estimate the moment coefficient in both upstream and downstream regions which is a precursor for the design of an active pitch angle control system. Also, an intelligent controller based the neural networks system is proposed to predict the optimum pitch angle control command for the blades actuators in order to increase the power output.

Although VAWTs have been in existence for many years, very limited research has been conducted with respect to these systems, in comparison with the extensive literature devoted to HAWTs [37]. Only recently, have VAWTs received increased analytical, numerical, and experimental attention; a trend that is attributable to their ability to achieve useful power production but with less noise [38]. This chapter provides a review of the literature on VAWTs, which is divided into two groups: (1) analytical and numerical studies in which the aerodynamic performance of VAWTs is explored, and (2) literature on the design of wind turbine pitch control systems.

\subsection{Analytical and numerical studies for investigating the aerodynamic of VAWTs}

Numerous analytical, numerical, and experimental methods have been employed for studying the flows around VAWTs in order to better understand their aerodynamic behavior at both with fixed and variable pitch angles [39]. The Double Multiple Stream-Tube model 
(DMST) was proposed by Paraschivoiu [40]. It is an analytical model based on the Blade Element Method (BEM). It is also commonly used to investigate the aerodynamic characteristics for VAWTs. Although the DMST model is slightly inaccurate, it is a starting point to predict the performance of VAWTs. Paraschivoiu used the DMST model to study the variable pitch angle VAWTs and indicated that the variable pitch angle strategies can improve self-starting capability, increase power coefficient peaks and reduce the vibration of blades by avoiding stall. Zhao et al [41] proposed a new blade pitching approach and used the DMST model to investigate the effect of blade pitching on power output of a VAWT. The results of this study displayed that the variable pitch technique increased the peak power coefficient by about $18.9 \%$ and enhanced the self-starting capability. Kavade and Ghanegaonkar used Single Stream Tube (SST) and Double Multiple Stream Tube (DMST) models to study the effect of best position of blade pitching on performance of a VAWT. It was found that the power coefficient and self-starting capability were improved using different pitch angles $\left(45^{\circ}\right.$ and $\left.15^{\circ}\right)$ and TSRs. Also, Jain and Abhishek [42] used DMST to predict the aerodynamic performance of a VAWT with dynamic blade pitching (sinusoidal blade pitching). Their work concluded that the pitch amplitudes should be high $\left(\approx 35^{\circ}\right)$ for TSRs below 0.5 and should be reduced to approximately $10^{\circ}$ for TSRs greater than 2.0.

High performance computing systems have recently been employed as a means of addressing a number of engineering problems. Computational Fluid Dynamics (CFD) is a powerful numerical tool for acquiring an understanding of the aerodynamic response of VAWTs through solving the Navier-Stokes equation. There are several studies that have been reported about the use of CFD for investigating the aerodynamics of VAWTs. Balduzzi et al. [43] examined some of the model parameters, such as the dimensions of domains, mesh sizes, turbulence models, etc., in order to examine their influence on the performance of a $2 \mathrm{D} \mathrm{H}$ type VAWT with a fixed pitch angle. Their CFD results agreed well with the experimental 
data. Chen et al. [3] used the ANSYS-Fluent CFD package to assess the performance of a VAWT by using sinusoidal pitching, which in turn changed the angle of attack. A $2 \mathrm{D}$ study was conducted on a three-bladed VAWT with an NACA 0018 airfoil. The results were compared to a fixed pitch VAWT, which revealed that blade pitching can improve the power efficiency. Also, the fluctuation in power output, rotation speed and torque output were suppressed. Li et al. [4] used the genetic algorithm in conjunction with CFD simulation to predict the optimum pitch angles. Their numerical results showed that the optimized blade pitches can increase the average power coefficients. Using the ANSYS-CFX package, Sumantraa et al.'s study [5] was focused on the effect of a preset pitch angle on the performance of an H-type VAWT. The CFX model simulated and analyzed three pitch angles (namely, $-6^{\circ}, 0^{\circ}$, and $+6^{\circ}$ ) with varied TSRs and wind speeds. For each pitch angle configuration, flow field characteristics were investigated, and the power curves were compared with each other. The authors concluded that the best performance occurred at a pitch angle of $-6^{\circ}$ for all TSRs and wind speeds. Sagharichi et al. [6] argued that the low performance of fixed pitch VAWT at high solidities could be enhanced by using the blade pitching technique. The effect of solidity on aerodynamic characteristics for a VAWT with fixed and variable pitches was investigated numerically based on a $2 \mathrm{D}$ incompressible turbulent flow CFD model.

Most numerical studies used experimental data collected on full-scale VAWTs with fixed blade to validate the obtained simulation results. Raciti Castelli et al. [44] investigated a flow field characteristics of a VAWT numerically and performed validation campaign for a Darrieus micro-VAWT through a systematic comparison with wind tunnel experimental data. Balduzzi et al. [45] validated their CFD simulation results for a VAWT against the experiment conducted in a wind tunnel with a full-scale VAWT, which is exactly the same geometry used in the CFD model. Vittecoq and Laneville [46] reported reliable experimental 
results in terms of instantaneous torque output of a Darrieus VAWT rotor. However, the experimental studies of a VAWT with variable blade pitch angles are quite rare in the open literature. Erickson et al. [9] explored the impact of sinusoidal pitch actuation on the performance of a high-solidity VAWT in a wind tunnel over a wide range of design and operational conditions. Their experimental work was conducted using a cam and control rod mechanism. The variable pitch VAWT achieved 35\% more power coefficient than the fixed blade configuration. Hantoro et al. [47] investigated experimentally the performance of an Htype VAWT in water using passive variable-pitch blade mechanisms. The results showed that the stall angles were reduced by using a passive variable pitch technique. As a result, the lift force was dominated during most the blade trajectories. Some experimental studies [48], [49] investigated the effect of different fixed blade pitch angles on the performance of Darrius VAWTs. These studies concluded that the blade pitching techniques can enhance the average power coefficient. However, all the previous experimental studies used simple techniques for blade pitching such as cams or leaving the blades free to pitch (passive control).

\subsection{Studies of wind turbine pitch control system design}

Numerous studies have suggested a variety of pitch angle control methods. The proportional-integral (PI) or proportional-integral-derivative (PID) based pitch angle controllers have been used for the power regulation. These linear control methods offer only limited performance, especially in the face of the uncertainties associated with the nonlinear dynamic properties of wind turbines [50]. A linear quadratic Gaussian (LQG) control method is also applied for the pitch angle control. Although it is a more robust method compared to the PID control method, its performance is also limited to be applicable to the nonlinear system model [50]. Sliding-mode control techniques have also been applied to the pitch angle control. These techniques provide robust performance but require an observer to estimate the aerodynamic torque and rotor acceleration [51]. Intelligent control techniques that employ 
approaches such as neural networks and fuzzy logic have also been proposed for the modeling and control of nonlinear dynamical systems [52]. These methods are powerful when the system contains high non-linearity due to effects such as strong wind turbulence. Tiwari and Babu [14] proposed an advanced pitch angle control strategy for variable speed wind turbines using Radial Basis Function Network (RBFN) and Feedforward based Back Propagation Network (BPN) algorithm to generate pitch angle. The performance of the proposed control system is compared to Fuzzy Logic Control (FLC) and ProportionalIntegral (PI) control techniques. The simulation results show that the proposed control strategy can reduce the fluctuations due to the wind speed variations in generated power. An Artificial Neural Network (ANN) can not only estimate a number of nonlinear functions based on information available for training but also provide a high degree of accuracy under specific system conditions [53]. All of the above research related to pitch control systems has been conducted for HAWTs. To the best of the author's knowledge, the published literature contains no studies related to intelligent control of the pitch angle for Darrieus VAWTs. Therefore, this thesis proposes the design of a novel intelligent pitch control system in order to improve the performance of a VAWT in terms of its power output.

Sargolzaei [54] applied ANNs for predicting the power coefficient for Savonius VAWTs that have six different shapes of rotor blades at different Reynolds numbers. The simulation results, which were compared with experimental data, revealed that use of the proposed ANNs technique provided a reasonable estimation of VAWT power coefficient. Hossain et al. [55] investigated the ability of the Fuzzy Expert System (FES) to predict the power generation of a small Hybrid (Darrieus and Savonius) VAWT compared to the experimental results. The results have demonstrated that the proposed FES is effective. Although these studies used artificial intelligent methods for only predicting the power output of VAWTs, 
there were no studies so far that used same proposed intelligent methods for improving the performance of VAWTs in terms of power output and self-starting capability.

The wind turbine performance can be optimized by predicting the optimum blade pitch angle in order to generate the highest value of torque. A key goal of this thesis is to design an intelligent blade pitch controller based on ANNs strategy for a small H-type VAWT. The proposed controller will generate blade pitch angle commands based on simulation data created from a dedicated CFD model which will be presented in Chapter 3.

\subsection{Methodology}

To investigate the effect of blade pitching technique on the performance of an H-type VAWT in terms of power output, a systematic approach is proposed in this section.

1. Analyze the aerodynamic characteristics of an H-type VAWT numerically using CFD method in both fixed and variable blade pitch angle configurations.

2. Use the knowledge in 1 to determine the optimum blade pitch angles that can improve the performance of an H-type VAWT.

3. Design a reliable intelligent pitch control system based on the optimum pitch angles obtained in 2.

4. Present a theoretical proof of stability of the proposed control system by modeling the H-type VAWT mathematically.

5. Validate all above steps experimentally by building a prototype of the H-type VAWT and testing it in different environmental conditions.

In this thesis, all the steps above are successfully realized. In order to investigate the performance of the H-type VAWT, a 2D H-type VAWT model is simulated numerically by using the ANSYS-Fluent CFD commercial software at different pitch angles. Both steady and transient solvers are adopted to predict the H-type VAWT performance by means of Multiple Reference Frame (MRF) and Sliding Mesh (SM) techniques, respectively. The k- $\varepsilon$ and k- $\omega$ 
turbulent models, which solve two extra transport equations for turbulence quantities are employed to describe turbulent flow over the Darrieus VAWT. The power coefficients of an H-type VAWT are obtained for both fixed and variable pitch angle configurations. For each blade, the optimum pitch angles which maximized the average power coefficients are also determined at each blade position. More details will be discussed in Chapter 3 .

Based on CFD results, a Hybrid active pitch control system, which combines MLP-ANN and PID controllers, is proposed in this thesis (Chapter 4) for each blade to investigate the effect of blade pitching on performance of an H-type VAWT in terms of power output and self-starting capability. In order to design a Hybrid pitch control system, a dynamic model of an H-type VAWT is also developed based on CFD results.

The stability analysis of control system based on the Lyapunov stability theory is carried out in Chapter 5. The Quadratic Lyapunov Function is used for stability analysis of nonlinear control system for an H-type VAWT.

In Chapter 6, an H-type VAWT experimental prototype is presented. Also, the Hybrid control system is implemented for each blade individually to set the desired (i.e., optimum) blade pitch angles. Extensive outdoor experiments were carried out to investigate the effect of blade pitching technique on the performance of an H-type VAWT as well as examine the effectiveness of proposed pitch control system. Moreover, the CFD results were validated in comparison with experimental results. The experimental results showed that the blade pitching technique can improve the performance of an H-type VAWT in terms of power output and self-starting capability. 


\section{Chapter 3}

\section{COMPUTATIONAL FLUID DYNAMICS (CFD) MODEL ${ }^{1}$}

\subsection{Introduction}

This chapter introduces the modeling of a 2D H-type VAWT using a CFD method. Even though wind tunnel tests are used to investigate the performance of VAWTs in terms of power output or self-starting capability, results often cannot provide a comprehensive and deep understanding of the complex aerodynamic behavior of VAWTs. Therefore, inexpensive solutions for performing the complex aerodynamic analysis are required. Computational Fluid Dynamics (CFD) is an ideal technique for solving fluid dynamic problems and analyzing systems related to fluid flow and heat transfer by means of computer-based simulations. Many engineering applications are investigated using CFD, for example [56]:

- Aerodynamics of aircraft and vehicles,

- Power plants,

- Turbomachinery,

- Electrical and electronic engineering applications.

The fluid governing equations can be defined by applying the laws of mechanics to a fluid. In order to address the fluid flow problem, CFD codes based on numerical algorithms not only can provide a large number of results to describe the physics of fluid flow but also can perform parametric studies to optimize the performance of equipment itself [57]. In addition, commercial CFD packages such as ANSYS contain interfaces that allow users to input the parameters of the problem and simulate the flow behaviour of the system.

\footnotetext{
${ }^{1}$ Published in Renewable Energy Journal [110]
} 


\subsection{The CFD model}

CFD techniques have recently been applied to study the aerodynamic behavior of VAWTs [37]. In the study presented in this thesis, ANSYS-Fluent was utilized for the simulation of both fixed and variable pitch angle 2D three-bladed H-Darrieus VAWT with NACA 0018 airfoil at different tip speed ratios in order to predict the H-type VAWT aerodynamic performance and investigate the self-starting capability. The flow filed around the rotor is also analyzed using both multiple reference frame (MRF) and the sliding mesh (SM) techniques. MRF is a steady-state computational fluid dynamics (CFD) modeling technique, while SM is a transient (unsteady) simulation technique. Therefore, MRF model is used in this study to calculate flow field parameters that can be used as initial conditions for the sliding mesh calculations.

Table 3.1. Main features of the Darrieus wind turbine analyzed in the present study.

\begin{tabular}{ll}
\hline \multicolumn{1}{c}{ Feature } & \multicolumn{1}{c}{ Value } \\
\hline Rotor radius $(\mathrm{R})[\mathrm{mm}]$ & 850 \\
Blade height $(\mathrm{H})(2 \mathrm{D})[\mathrm{mm}]$ & 1 \\
Blades number $\left(N_{b}\right)[-]$ & 3 \\
Blade profile $[-]$ & NACA 0081 \\
Chord $(c)[\mathrm{mm}]$ & 246 \\
Pitch angle $(\beta)\left[^{\circ}\right]$ & $-6,-4,0,4,6$ \\
Azimuth angle $(\theta)\left[^{\circ}\right]$ & 0 to 360 \\
Tip speed ratio $(\lambda$ or TSR) $[-]$ & $1,1.7,2,2.5,3.3$ \\
Solidity $(\sigma)[-]$ & 0.14 \\
\hline
\end{tabular}

Table 3.1 shows the main dimensions and parameters of the H-type VAWT CFD model, which are based on previous studies [43][5]. For each TSR $(1,1.7,2,2.5$, or 3.3), the H-type VAWT model is simulated at each pitch angle $\left(\beta=-6^{\circ},-4^{\circ}, 0^{\circ}, 4^{\circ}\right.$, and $\left.6^{\circ}\right)$ over ten or more of cycles to allow for convergence. The results of the last revolution are chosen for the analysis. Because ANSYS-Fluent can be used to obtain the moment coefficient $\left(C_{m}\right)$ for each blade, the maximum moment coefficient $\left(C_{m, \max }\right)$ was determined at each azimuth angle in order to 
define the optimum blade pitch angle $\left(\beta_{\text {opt }}\right)$, and hence, the power coefficients $\left(C_{p}\right)$ were calculated for all cases.

\subsubsection{ANSYS pre-processing}

The ANSYS Workbench Pre-processing tools are used to define the H-type VAWT geometry. Pre-processing includes building the 2D H-type VAWT model within the flow domains and then creating meshes in ANSYS-Fluent Meshing mode. This chapter presents more details about the H-type VAWT modeling by using CFD.

\section{A. Computational domains}

Figure 3.1 shows the main computational domains that are generated by the ANSYS Design Modeller. The H-type VAWT CFD model used in the study presented in this thesis consists of three main subdomains: a stationary subdomain, a rotating subdomain, and a blade subdomain:

- Stationary domain: it should be large enough to avoid a solid blockage effect of the lateral boundaries and describe a development of the wake. Therefore, the dimensions of the domain are 40 rotor diameters upstream (L1),100 rotor diameters downstream (L2), and 60 rotor diameters width (W) [43].

- Rotating domain: it is recommended to be very small in order to better describe the vorticity accurately and avoid undesirable disturbances generated at the interface. The radius of the circular domain $(2.5 m)$ is almost three times the rotor radius $(R)$ [43].

- Blade sub-domain: in order to study the boundary layer of the airfoils, the mesh around these airfoils should be finer. Therefore, each blade has a circular sub-domain with diameter equal to $0.4 m$. 


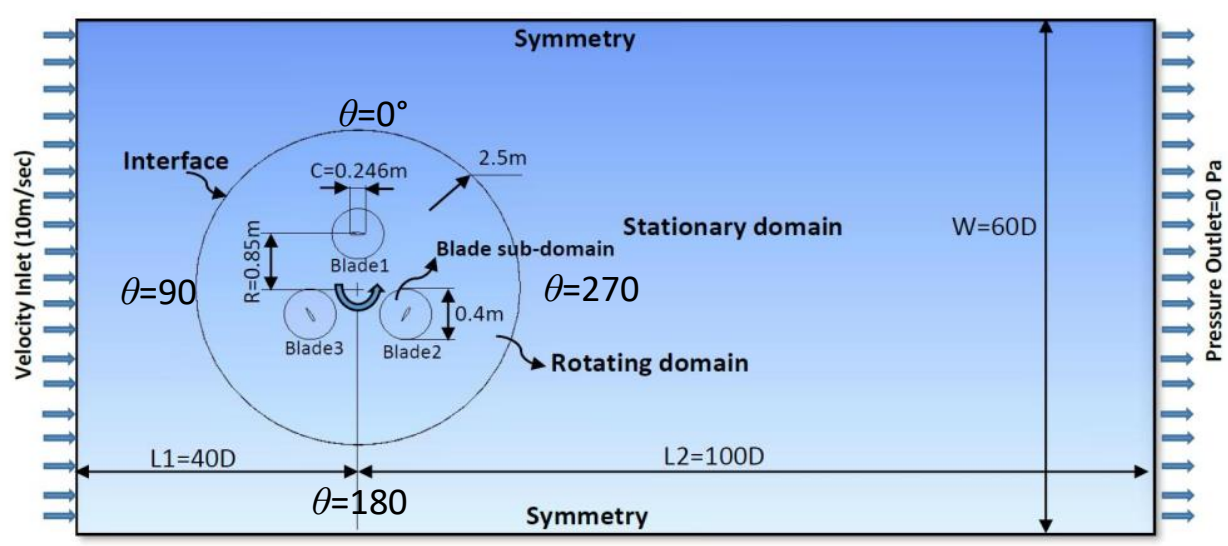

Figure 3.1. Main computational subdomains used in ANSYS Design Modeler.

\section{B. Grid generation}

The accuracy of the model results is sensitive to the size and distribution of the mesh. In the 2D simulations for this study, unstructured mesh is generated using ANSYS Workbench and the mesh is characterized by triangular elements. In order to resolve the boundary layer of the airfoils, very fine mesh is created around the blades (i.e., blade sub-domain) as can be seen in Fig. 3.2 (a) and (b). Particularly, the number of layers around each blade and the thickness of the first layer were equal to 50 and $3.561 \times 10^{-5} \mathrm{~m}$, respectively to achieve y+ values lower than 5 and capture the viscous sub-layer.

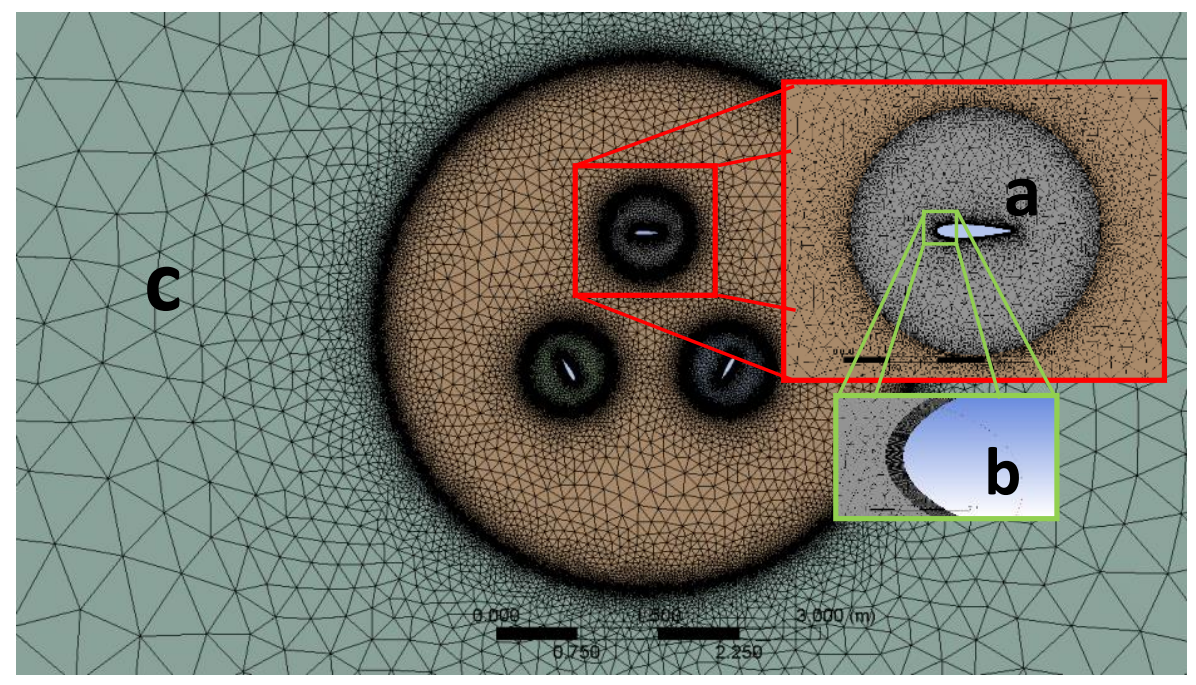

Figure 3.2. Mesh of the Darrieus turbine modeled for this study.

A dimensionless wall distance $(y+)$ is commonly used in boundary layer theory and in defining the law of the wall. $y+$ can be defined in the following way: 


$$
y^{+}=\frac{\left(u_{*}\right) y}{v}
$$

where $u_{*}$ is the friction velocity at the nearest wall, $y$ is the distance to the nearest wall and $v$ is the local kinematic viscosity of the fluid [58]. The near wall flow can be divided into three sublayers [59]:

1. $y+<5$ : viscous sublayer

2. $5<y+<30$ : buffer sublayer

3. $y+>30$ : fully turbulent sublayer.

In this study, the quality of near-blade mesh was investigated using $y+$ values. The average of $y+$ is around 1.5. Hence, the region around the airfoils is in the viscous sublayer which is recommended for accurate results [59]. To reduce the computational cost of the overall CFD model, a close-to-equilateral coarse mesh is generated in the stationary domain, as shown in Fig. 3.2 (c).

The number of nodes and elements, aspect ratio, and skewness for the 2D H-type VAWT simulations are listed in Table 3.2. Because of the limited mesh size in the ANSYS product license accessible, the total number of mesh elements was chosen to be slightly smaller than the recommended number of elements proposed in [43]. However, the skewness and aspect ratio indicated that the quality of the current mesh is acceptable to describe the flow around the Darrieus rotor. Skewness and aspect ratio are significant measures of mesh quality where the skewness value should be less than 0.9 and aspect ratio less than 50 for most applications [58].

Table 3.2. Mesh features for current VAWT model.

\begin{tabular}{ll}
\hline \multicolumn{2}{c}{ Mesh features } \\
\hline Number of nodes & 167443 \\
Number of elements & 236907 \\
Aspect ratio & 54.222 \\
Skewness & 0.71 \\
\hline
\end{tabular}




\subsubsection{ANSYS-Fluent solver}

To predict the H-type VAWT performance, steady and transient solvers were applied by using both multiple reference frame (MRF) and sliding mesh (SM) techniques, respectively. ANSYS-Fluent uses many turbulence models based on Reynolds-averaged Navier-Stokes (RANS) equations to represent the turbulent properties of the flow. Typical examples of such models are the k- $\varepsilon$ and $k-\omega$ turbulence models in their different forms including two extra transport equations that are solved for the turbulence kinetic energy $(k)$ and its dissipation rate ( $\varepsilon$ or $\omega$ ). This thesis used the Shear-Stress Transport (SST k- $\omega$ ) model that is one of the k- $\omega$ turbulence model-proposed by Menter in 1993 [60]. It combines two turbulence models via blinding functions: a standard k- $\omega$ turbulence model to treat the near-wall region and a transformed k- $\varepsilon$ turbulence model to study the outer region. Hence, it is more accurate for a wide range of boundary layer flows with pressure gradient [61]. The boundary conditions are shown in Fig. 3.1. The inlet and outlet are defined as an air velocity inlet $\left(u_{\infty}=10 \mathrm{~m} / \mathrm{s}\right)$ and pressure outlet (atmospheric pressure), respectively. Also, turbulent intensity $(I)$ and turbulent length scale $(l)$ are defined $1 \%$ and $5 \%$ of the width $(\mathrm{W})$ for both the inlet and outlet boundaries, respectively. Two symmetry boundary conditions are applied for both side walls. No-slip shear condition is assumed for the blades. For solution methods in ANSYS-Fluent:

- $\quad$ The COUPLIED scheme is applied.

- $\quad$ Least-squares Cell-based is chosen as the gradients of solution variables.

- $\quad$ Pressure-based solver with the standard pressure are selected as a pressure equation.

- $\quad$ Second order upwind are set as a discretization scheme.

These methods are usually used for VAWT CFD modeling [43]. Moment, lift, and drag coefficients are monitored. Using an accurate time step size can reduce the discretization errors and increase the stability of calculation [43][62]. Table 3.3 shows different time step sizes $(\Delta t)$ that are used herein for each simulation. 
Table 3.3. Operating values for current CFD model.

\begin{tabular}{cccc}
\hline TSR & $\begin{array}{c}\boldsymbol{\omega}_{\boldsymbol{r}} \\
(\mathbf{r a d} / \mathbf{s e c})\end{array}$ & $\begin{array}{c}\text { Time step size }\left(\mathbf{s} / \mathbf{1}^{\mathbf{0}}\right) \\
\boldsymbol{2} \boldsymbol{2} \boldsymbol{\pi} /\left(\boldsymbol{\omega}_{\boldsymbol{r}} \times \mathbf{3 6 0}\right)\end{array}$ & $\begin{array}{c}\text { number of time steps } \\
\text { (revolutions) }\end{array}$ \\
\hline $\mathbf{1}$ & 11.8 & 0.001484 & $\geq 3600$ \\
$\mathbf{1 . 7}$ & 20 & 0.000873 & $\geq 3600$ \\
$\mathbf{2}$ & 23.53 & 0.000742 & $\geq 3600$ \\
$\mathbf{2 . 5}$ & 29.41 & 0.000593 & $\geq 3600$ \\
$\mathbf{3 . 3}$ & 38.82 & 0.00045 & $\geq 3600$ \\
\hline
\end{tabular}

For each simulation, the moment coefficient for each blade $C_{m 1}, C_{m 2}$, and $C_{m 3}$ is stored at each rotor position. The average of moment coefficients $\left(\bar{C}_{m}\right)$ are determined for all blades at each azimuth angle by:

$$
\bar{C}_{m}=\frac{\sum_{i=1}^{n=3} C_{m i}}{n}
$$

The rotor power coefficient $C_{p}$ can be calculated by the following equation:

$$
C_{p}=T S R \times \bar{C}_{m, \text { total }}
$$

where $\bar{C}_{m, \text { total }}$ is the average of moment coefficients $\bar{C}_{m}$ during one cycle. The optimum pitch angle $\left(\beta_{\text {opt }}\right)$ can be obtained by defining the maximum torque coefficient $\left(C_{m, i, m a x}, i=1,2,3\right)$ for the TSR value ranging from 1 to 3.3 .

\subsection{Validation}

The CFD model was validated through comparison with published wind tunnel experimental and CFD results for an H-type VAWT [43]. Because the experimental data are available only for fixed pitch angle, the CFD simulation results were validated, herein, only for the fixed pitch angle case. In this thesis, the 2D H-type VAWT model has the same geometry size of the 2D H-type VAWT model with a fixed pitch angle that was simulated using a CFD method and reported by Balduzzi et al. [43] but with some differences, such as the shape of computational domains and the number of elements. To reduce the computational cost, some parameters, such as the number of iterations and the time step size, were also reduced in the ANSYS-Fluent setup used in this thesis. 


\subsection{CFD simulation results}

In the study presented in this thesis, the analysis is carried out for pitch angles of $\beta=-6^{\circ},-4^{\circ}$, $0^{\circ}, 4^{\circ}$, and $6^{\circ}$; TSRs of $\lambda=1,1.7,2,2.5$, and 3.3; and mean wind speed of $u_{\infty}=10 \mathrm{~m} / \mathrm{s}$. Figure 3.3 shows the power coefficients $\left(C_{p}\right)$ curves at the different pitch angles, including the fixed pitch angle case $\left(\beta=0^{\circ}\right)$. The predicted curves are then compared with other published experimental and CFD results with respect to the power coefficient.

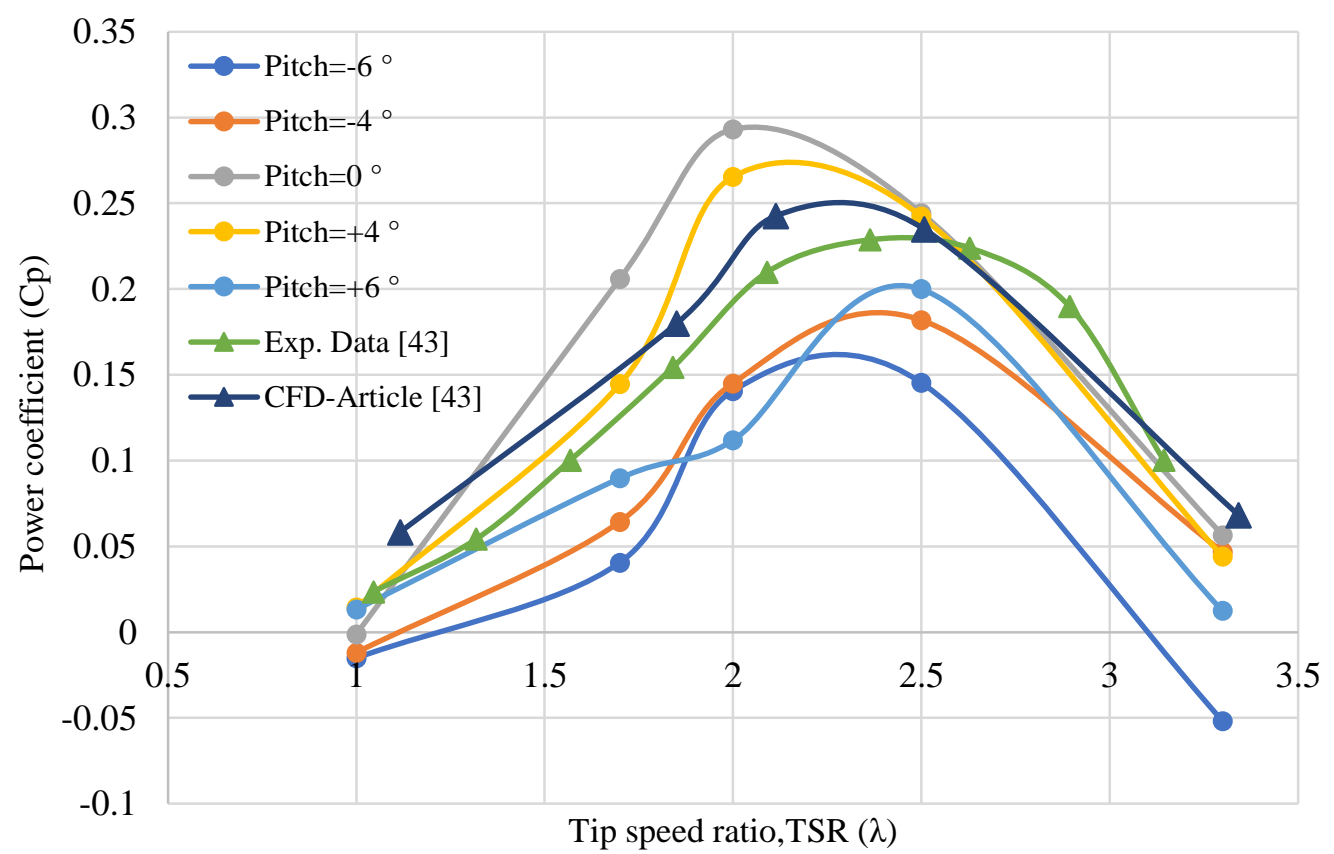

Figure 3.3. Comparison of the CFD results for this study with other published experimental and CFD results, with respect to the power coefficient versus the TSR at different pitch angles.

Although the wind tunnel prototype (i.e., experimental study) is a 3D model, the predicted power curves obtained for this study follow a trend that is similar to the published experimental and CFD data. For the fixed pitch angle case, good agreement among all results was observed at TSR values greater than 2.5. However, the CFD models developed for this thesis shows slightly overestimated results of the peak value of $C_{p}$ that it may be due to the accuracy of the proposed turbulence model in prediction of aerodynamic performance. Also, a discrepancy can be noted in $C_{p}$ values at low TSR of $\lambda=1$ between CFD and experimental 
results. This behavior is probably due to the differences evident between the angle of attack variations in the computational and experimental conditions.

Figure 3.3 also shows that, with negative and zero pitch angles, negative power coefficients are predicted at a low TSR of $\lambda=1$ because of a negative generated torque that might be a result of overestimating the angle of attack using CFD method. It could be observed that the $C_{p}$ peaks for all curves occur at TSRs between 2 and 2.5 (i.e., optimal TSRs or $\lambda_{\text {opt }}$ ). Also, the $C_{p}$ curve at the pitch angle of $\beta=+4^{\circ}$ was better than all other the pitch angles at all TSRs.

For the positive pitch angles, the power coefficient at low TSR of $\lambda=1$ was increased by around 12 percent compared to the fixed pitch angle. This means that an enhancement in the self-starting capability of an H-type VAWT can be achieved using the blade pitching technique. Table 3.4 shows the power coefficients $\left(C_{p}\right)$ values at different TSRs and pitch angles.

Table 3.4. Power coefficients $\left(C_{p}\right)$ at different TSRs and pitch angles.

\begin{tabular}{cccccc}
\hline \multirow{2}{*}{ TSR } & \multicolumn{5}{c}{ Pitch angles $\left(^{\circ}\right)$} \\
\cline { 2 - 6 } & $\mathbf{- 6}$ & $\mathbf{- 4}$ & $\mathbf{0}$ (fixed) & $\mathbf{4}$ & $\mathbf{6}$ \\
$\mathbf{1}$ & -0.01519 & -0.01201 & -0.00125 & 0.014456 & 0.013229 \\
$\mathbf{1 . 7}$ & 0.040504 & 0.0643 & 0.205944 & 0.144615 & 0.089795 \\
$\mathbf{2}$ & 0.140547 & 0.145001 & 0.292979 & 0.265298 & 0.111846 \\
$\mathbf{2 . 5}$ & 0.145308 & 0.181745 & 0.244328 & 0.242333 & 0.199989 \\
$\mathbf{3 . 3}$ & -0.05182 & 0.046705 & 0.056599 & 0.044126 & 0.012437 \\
\hline
\end{tabular}

In order to investigate the effect of blade pitching technique on the performance of an $\mathrm{H}$ type VAWT at low TSRs, the torque characteristics of the 2D H-type VAWT model were examined for all pitch angles at TSR of $\lambda=1$.

Figure 4.3 shows the single blade torque coefficients (blade 1) for five pitch angles over the last revolution at a low tip speed ratio TSR of $\lambda=1$. For the upwind region $\left(\theta=0^{\circ}-180^{\circ}\right)$, which is shown in Fig.3.1, peaks of torque for all cases occur at azimuth angles less than $30^{\circ}$. The torque curves with positive pitch angles are slightly wider than other curves with zero and 
negative pitch angles. This means that a higher torque was generated because the stall of the airfoil was delayed and consequently the flow separation was also delayed.

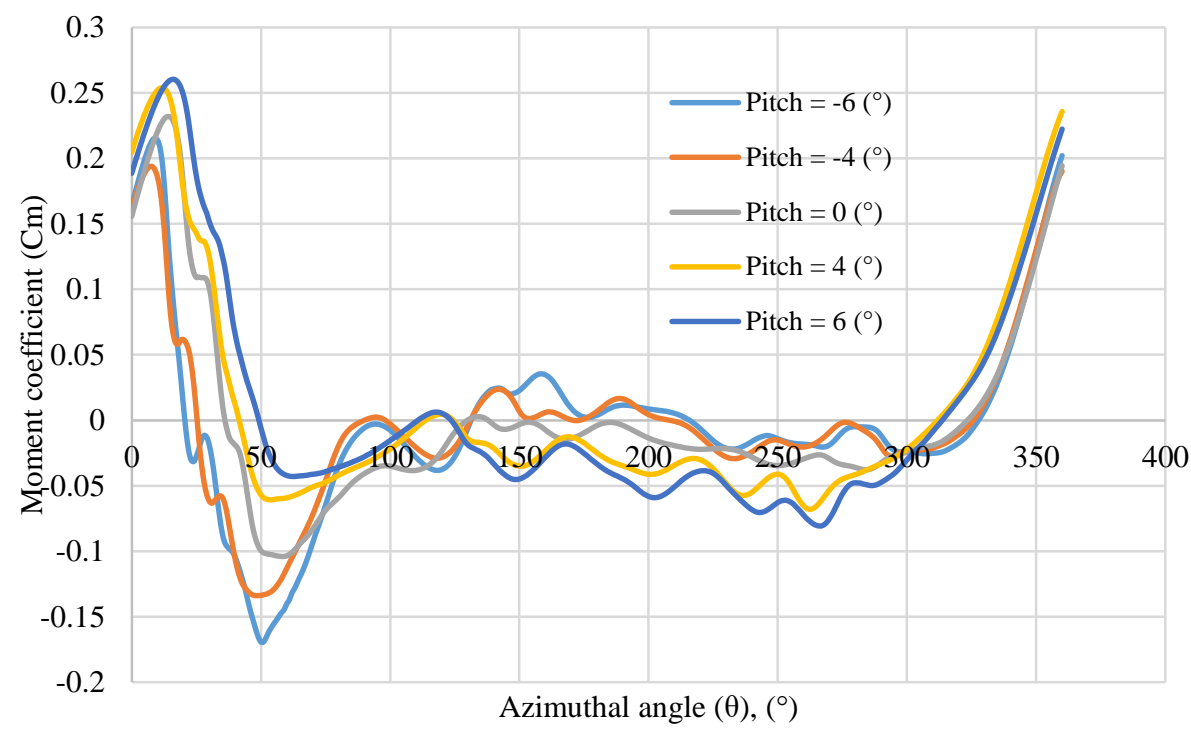

Figure 3.4. Moment coefficient variations for the blade 1 at low TSR of $\lambda=1$ with different pitch angles.

However, since the flow detached too early (approximately at $\theta=20^{\circ}$ ) for the fixed and negative pitch angles, the stall vortex was created leading to a noticeable pressure difference across the upper and lower surfaces of the blade. The stall vortex can dramatically affect the downwind torque production. This behavior might be used to also explain the sudden drop of torque production in these curves after the peak at $\theta \approx 30^{\circ}$. It can be seen that the predicted torque in the downwind zone $\left(\theta=180^{\circ}-325^{\circ}\right)$ is mostly negative for all the pitch angles. This could be a result of the higher intensity and proximity of the stall vortex which is generated from upstream blades. Figure 3.5 shows the optimum pitch angles $\left(\beta_{\text {opt }}\right)$ that provide the highest moment coefficients at different TSRs as a function of the position angle $(\theta)$. 

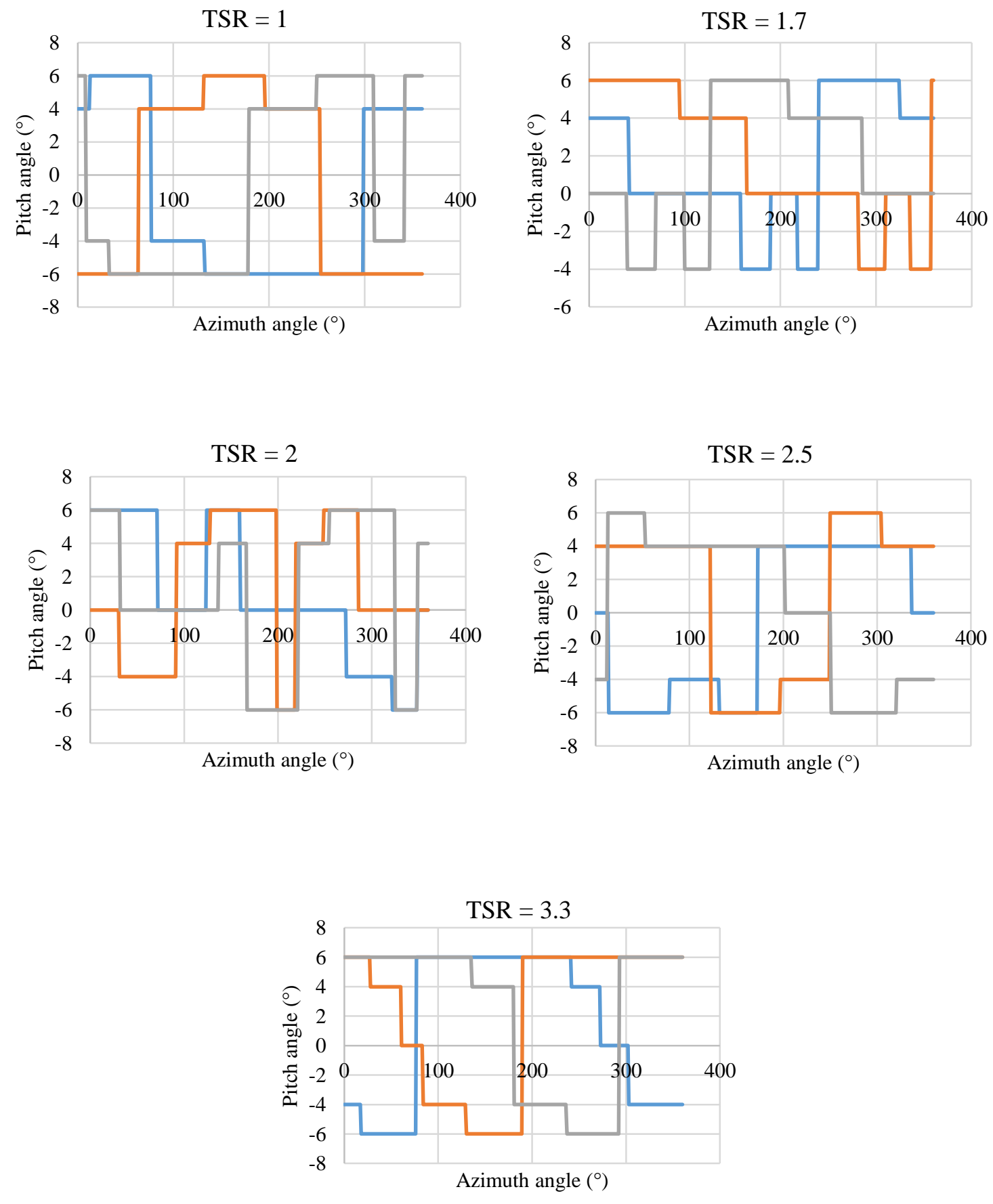

$\longrightarrow \mathrm{B} 1-\mathrm{B} 2-\mathrm{B} 3$

Figure 3.5. Optimum pitch angles for each blade of the H-type VAWT at different TSRs. 


\subsection{Conclusion}

In the CFD models developed for this thesis, the Computational Fluid Dynamics (CFD) solver is employed to analyze the performance of a 2D variable pitch angle H-type Darrieus VAWT with NACA0018 airfoil at different tip speed ratios (TSRs). In addition, multiple reference frame MRF and sliding mesh techniques available from ANSYS-Fluent were adopted to examine the 2D flow physics of the H-type VAWT with different pitch angles. For each case examined, the power coefficient $C_{p}$ is calculated and compared to published experimental and CFD results. Moreover, the effect of blade pitching technique on both performance and self-starting capability of a three-bladed straight H-Darrieus VAWT are investigated. Individual blade pitching can be a powerful strategy to improve the performance of the H-type VAWT by delaying the dynamic stall. Also, the poor self-starting capability of the H-type VAWT can be enhanced at low TSRs. The turbine's dynamic behavior was described properly through 2D CFD simulations. The optimum pitch angles, which maximized the moment coefficients, were determined at different TSRs. The results obtained from the CFD simulation model will be used in the next chapter to design an intelligent blade pitch controller for the three-bladed H-type Darrieus VAWT in order to improve the power output in real time operation. 


\section{CHAPTER 4}

\section{MODELING OF H-TYPE VAWT ${ }^{2}$}

Examining the effect of pitch angle on VAWT power output required the building of a dynamic model of the VAWT. Several studies [63][64][65] describe dynamic wind turbine models, but their primary focus was about the modeling of horizontal axis wind turbines (HAWTs). This chapter provides the dynamic model of an H-type VAWT. The full Matlab/Simulink model for an H-type VAWT is shown in Fig. A1 in Appendix A.

The wind turbine's central purpose is to capture the kinetic energy of wind and convert it to electrical energy [66]. Figure 4.1 is a block diagram of the H-type VAWT considered in this thesis, which was based on the HAWT model described in [67].

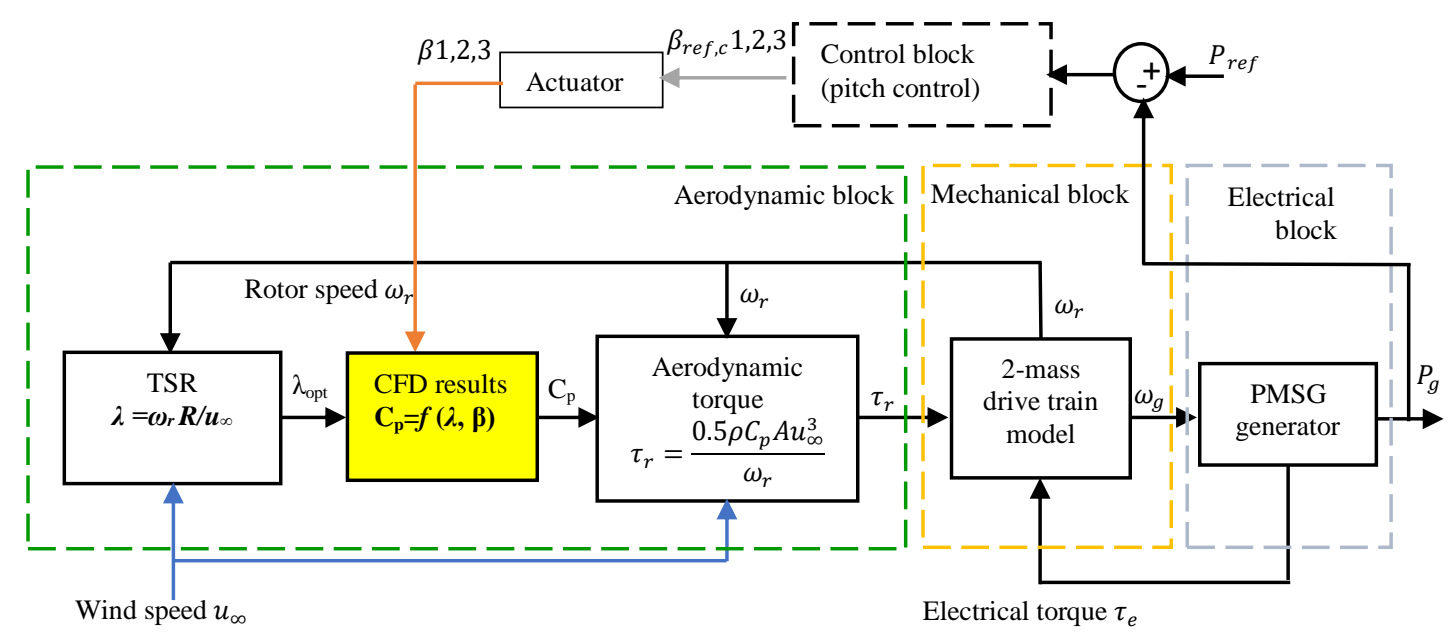

Figure 4.1. Block diagram for an H-type VAWT model [63].

The main wind turbine system consists of the following components:

1. Plant

- Aerodynamic block: Turbine rotor and blades

- Mechanical block: Shaft and gearbox unit (drive-train)

- Electrical block: Induction generator

${ }^{2}$ Published in Renewable Energy Journal [110] 
2. Control block (Pitch angle control system)

\subsection{Plant (H-type VAWT)}

The components of the H-type VAWT system as shown in Fig. 4.1 (i.e., aerodynamic, mechanical and electrical blocks) will be discussed in the next sections.

\subsubsection{Aerodynamic model (rotor model)}

Wind speed model: Wind speed model is not a part of wind turbine model. However, it is required for calculating the power output of wind turbines. Wind speed is usually modeled by four wind components; constant ( i.e., mean wind speed), ramp, gust and noise [64]. In this thesis, constant wind component is selected to represent wind speed $u_{\infty}$. The constant wind speed, which is considered as a uniform wind speed in this thesis, is the same as the inlet mean wind speed in the CFD model (i.e., $u_{\infty}=10 \mathrm{~m} / \mathrm{s}$ ) (see Section 3.2.2).

The power extracted from the wind can be expressed as follows [68]:

$$
P_{W}=\frac{1}{2} \rho A u_{\infty}^{3}
$$

where $\rho$ is the air density $\left(1.225 \mathrm{~kg} / \mathrm{m}^{3}\right), A$ is the swept area (rotor diameter $\mathrm{D} \times$ blade length $\mathrm{H}$ ) in $\mathrm{m}^{2}$, and $u_{\infty}$ is the uniform wind speed in $\mathrm{m} / \mathrm{s}$. In fact, only a fraction of this power can be captured by the turbine, representing the mechanical power from the wind turbine rotor $\left(P_{m}\right)$, defined as,

$$
P_{m}=\frac{1}{2} \rho A u_{\infty}^{3} C_{p}(\lambda, \beta)
$$

where $C_{p}$ is the power coefficient. Theoretically, the maximum limit of $C_{p}$ is 0.5926 under ideal conditions (Betz limit) [63].

Based on the CFD results in Chapter 3, it can be seen that the power coefficient of the Htype VAWT is a function of tip speed ratio $(\lambda)$ and blade pitch angle $(\beta)$, including the fixed 
pitch angle case $\left(\beta=0^{\circ}\right)$. This relationship represented by the surface in Fig. 4.2. The CFD results are also used to determine the optimum blade pitch angles $\left(\beta_{\text {opt }}\right)$ of the H-type VAWT and their maximum power coefficients $\left(C_{p, \max }\right)$ at different TSRs. To keep the power coefficient $C_{p}$ close to its maximum values $\left(C_{p, \max }\right)$ at a given wind speed, the corresponding TSR should also be retained close to its optimal value $\left(\lambda_{\text {opt }}\right)$, which is in the range of $2-2.5$, as shown in Fig. 4.2. The CFD results are then mapped to the aerodynamic model of the H-type VAWT rotor (i.e., "aerodynamic block" in Fig. 4.1), which is used to calculate the aerodynamic torque of the rotor $\left(\tau_{r}\right)$.

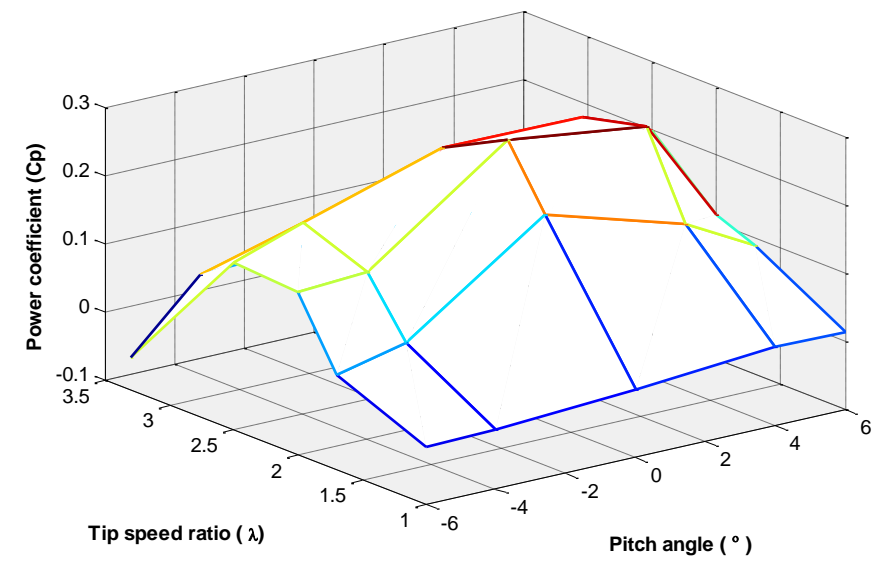

Figure 4.2. The H-type VAWT Power coefficient surface.

From Eq. (1.5), the amount of aerodynamic torque of the rotor $\left(\tau_{r}\right)$ in $N \cdot m$ is given by the ratio between the mechanical power extracted from the wind turbine $\left(P_{m}\right)$, in watt, and the turbine rotor speed $\left(\omega_{r}\right)$, in $\mathrm{rad} / \mathrm{s}$, as follows.

$$
\tau_{r}=\frac{P_{m}}{\omega_{r}}
$$

\subsubsection{Mapping of the H-type VAWT rotor (aerodynamic block)}

The aerodynamic models of the H-type VAWT rotor for the cases of both fixed and variable pitch angle cases are derived based on the CFD results in Chapter 3.

Developing an accurate mathematical model of a nonlinear physical system such as the VAWT can be complicated [69] as discussed in Section 1.5 in Chapter 1. Therefore, input- 
output mapping is proposed in this thesis to emulate the nonlinear system behavior [70]. Although there is no specific method to parameterize nonlinear dynamic systems, Artificial Neural Networks (ANNs) have been proposed as alternative techniques of system identification to model nonlinear systems [71]. The quality of data describing the plant I/O relationship is crucial if ANNs are used to model a nonlinear system from which such data is extracted. The purpose of mapping is to minimize the error between the predicted output from the ANNs and the actual output of the system. This error $e$ at $k+1$ can be expressed as follows [72]

$$
e(k+1)=\left|\hat{y}_{p}(k+1)-y_{p}(k+1)\right|
$$

where $\hat{y}_{p}(k+1)$ is the predicted output and $y_{p}(k+1)$ is the actual output.

In this research, the rotor part of the H-type VAWT is mapped using both a look-up table (LUT) and a multilayer perceptron artificial neural network (MLP-ANN) for cases involving fixed and variable pitch angles, respectively. The rotor parameters are available from the CFD results, such as power coefficients $\left(C_{p}\right)$, TSRs, azimuth angle $(\theta)$, and pitch angles $(\beta)$, are used as input and output data.

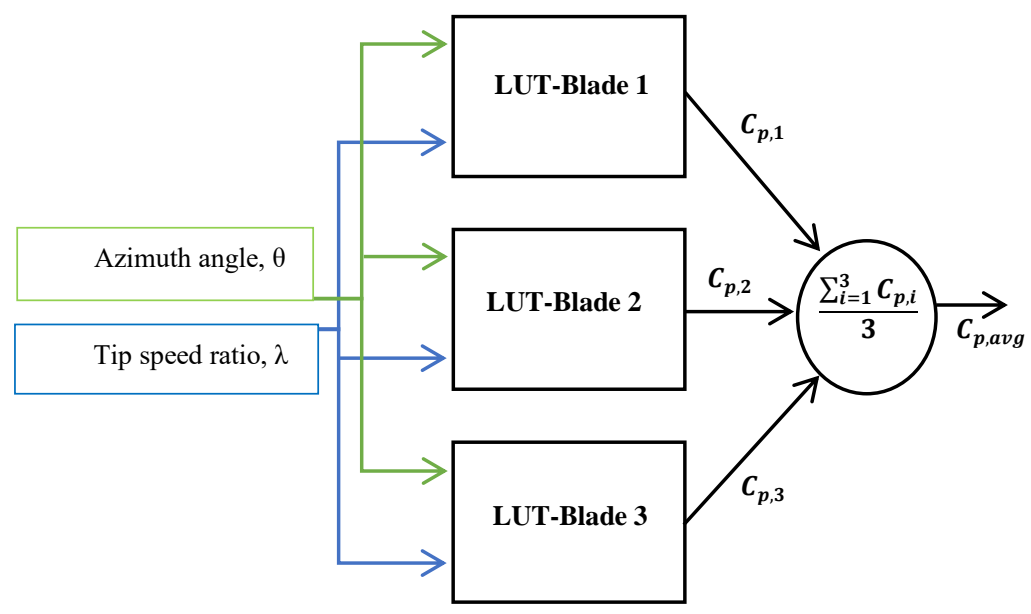

Figure 4.3. LUT-mapping of the power coefficient $\left(C_{p}\right)$ for a fixed pitch angle VAWT rotor. 


\section{A. Mapping of a fixed pitch angle H-Type VAWT rotor}

In order to estimate the average power coefficients $\left(C_{p, a v g}\right)$ for a fixed pitch angle H-type VAWT (i.e., $\beta=0^{\circ}$ ) over three blades, a look-up table (LUT) is implemented for each blade using two types of CFD input ( $\theta$, TSR) and one type of CFD output $\left(C_{p}\right)$, as illustrated in Fig. 4.3. The average power coefficient $\left(C_{p, a v g}\right)$ is used for determining the aerodynamic torque of the H-type VAWT rotor $\left(\tau_{r}\right)$ in Eq. (4.3).

\section{B. Mapping of a variable pitch angle H-type VAWT rotor}

Although an LUT can provide reasonably accurate results through linear interpolation, it is difficult to extend its use for more than two inputs. In the variable pitch angle H-type VAWT rotor model, the input consists of the TSR, $\theta$, and the optimum pitch angles $\left(\beta_{\text {opt }}\right)$, while the output is only the maximum power coefficient $\left(C_{p, \max }\right)$ for each blade. In this case, the MLPANN is utilized in order to approximate the H-type VAWT nonlinear system with a variable pitch angle.

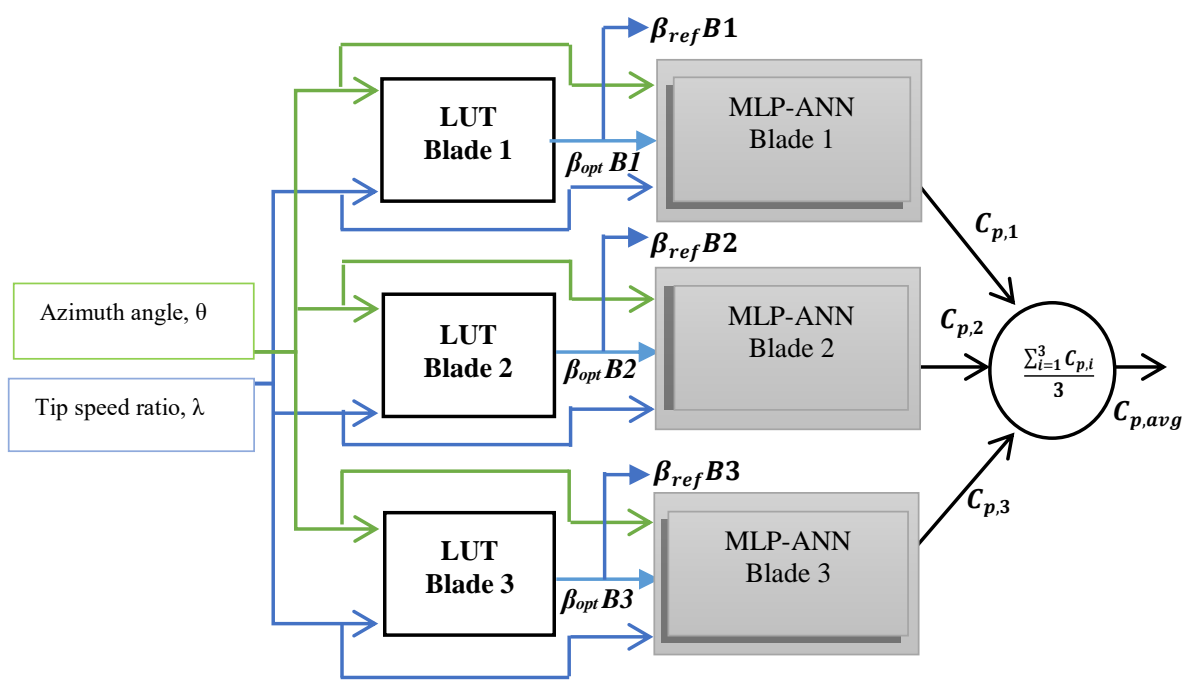

Figure 4.4. MLP-ANN mapping of the power coefficient $\left(C_{p}\right)$ for a variable pitch angle VAWT rotor.

The MLP-ANN output is an estimate of the power coefficient for each blade at different operating conditions. Specifically, MLP-ANN is trained using I/O data obtained from the CFD 
simulations in order to describe the aerodynamic properties of the rotor of the H-type VAWT, which represent a nonlinear relationship between the input and the output.

ALUT is also employed for estimating the optimum pitch angles for each blade $\left(\beta_{\text {opt }}\right)$, which is then used as one of the MLP-ANN input, as shown in Fig. 4.4. These angles will be also used in Section 4.2 as reference signals for the pitch angle control system (see Fig. 4.6).

\subsubsection{Drive-train model}

A drive-train can be described as a multi-mass system such as a 6-mass, 3-mass, 2-mass or single-mass model [73]. A 2-mass model to represent the dynamics of a turbine drive-train has been proposed and was shown to be accurate [73]. Both the turbine rotor and generator inertias are taken into account to model the drive train as 2-mass. The mathematical model for a 2mass drive train H-type VAWT will be discussed in Chapter 5. The drive-train parameters applied in Matlab/Simulink model, are listed in Table A1 in Appendix A.

\subsubsection{Model of Permanent Magnet Synchronous Generator (PMSG)}

A PMSG is usually utilized in a wind energy conversion system (WECS) because it has some advantages such as better reliability, lower maintenance and higher efficiency [74]. A fully developed permanent magnet synchronous generator model available in the Matlab/ Simulink tool has been adopted. For this research, the main parameters of the PMSG are listed in Table A2 in Appendix A. More details about modeling of the PMSG based on the d-q synchronous reference frame are also provided in Section A1 in Appendix A.

\subsubsection{Reference and actual plants}

The MatlablSimulink model presented in this thesis, which consists of two plants; reference and actual. The reference plant is implemented to generate the reference signals based on the CFD results such as power reference $\left(P_{r e f}\right)$ and reference (optimum) pitch angles $\left(\beta_{r e f}\right)$. 
However, the actual electrical power output $\left(P_{g}\right)$ is produced by the actual plant. Also, the actual plant receives the pitch control signals while the reference plant uses the pitch angles obtained from the CFD results.

\subsubsection{Pitch actuators}

For each blade, pitch servo is used to set the blades into the required position by adjusting the rotation of the blades around the longitudinal axes. The actuator is modeled as an integrator as shown in Fig. 4.5.

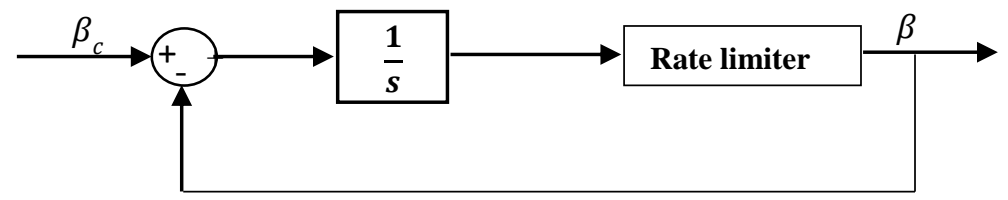

Figure 4.5. Blade actuator block diagram.

\subsection{Proposed H-type VAWT blade pitch control system}

Because of high nonlinearity of wind turbine dynamics due to the wind variations, control systems are implemented to maximize and regulate their power output. Many types of control system are applied for wind turbines such as blade pitch and generator torque control systems [75]. This research focuses on the effect of blade pitching on performance of the H-type VAWT in terms of power output.

For an H-type VAWT the output power may change dramatically as a result of a small change in the pitch angle [76]. Hence, the performance of an H-type VAWT can be improved by adjusting the pitch angle of the blades. The H-type VAWT model shown in Fig. 4.1 is nonlinear because the rotor efficiency, which is dependent on the rotor speed, the wind speed, and the blade pitch angle, is highly nonlinear. Therefore, linear control methods alone are not usually sufficient for ensuring the desired performance of an H-type VAWT. Also, adaptive or robust nonlinear control methods can be challenging to develop for an H-type VAWT pitch control because of the complex model in Fig. 4.1. ANN methods are able to characterize any 
complex nonlinear dynamic system with a certain degree of accuracy according to StoneWeiestrass universal approximation theorem [77]. Some studies [78][79] attempted to combine ANNs with conventional controllers such as PI or PID to develop a stable and robust control system for handling nonlinear system dynamics.

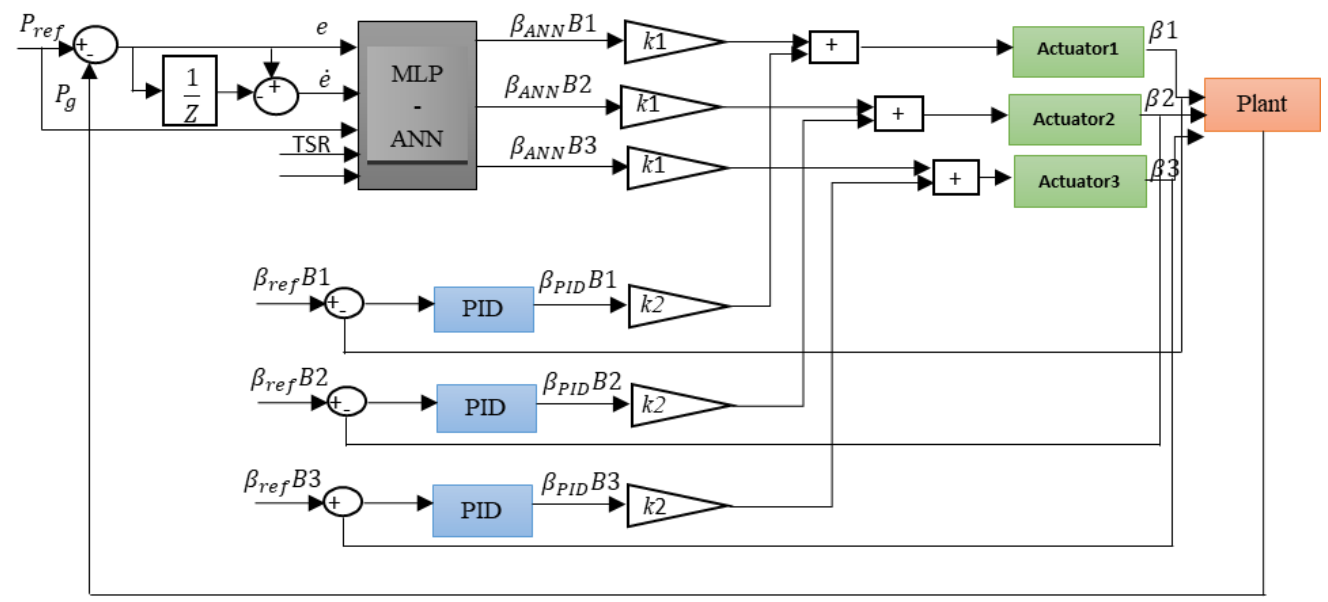

Figure 4.6. Proposed blade pitch control system block diagram.

The electrical power-tracking error $(e)$ is used widely as an input in pitch control system [80]. As shown in Fig. 4.6, the error $(e)$ is defined in terms of the reference and actual electrical power generation $\left(P_{g}\right)$ as follows:

$$
e=P_{\text {ref }}-P_{g}
$$

In this thesis, the three blades of the H-type VAWT are controlled individually using both global ANN and PID controllers (referred to as a Hybrid controller), as shown in Fig. 4.6. Multiple-input multiple-output (MIMO) MLP-ANN is proposed for designing an active intelligent blade pitch control system for an H-type VAWT. The error $(e)$, its derivative $(\dot{e})$, the reference power $\left(P_{r e f}\right)$, TSR, and the azimuth angle $(\theta)$ are introduced as input to MLPANN controller, while the output consists of the pitch angle additive command to supplement the linear control command for each blade. Furthermore, the signals received by the actuators are the sum of signals coming from both the MLP-ANN and PID controllers; $k 1 \%$ of the 
control command comes from the MLP-ANN controller and $k 2 \%$ comes from the PID controller. $k 1$ and $k 2$ are tuneable parameters.

\subsubsection{Using an MLP-ANN for mapping of a variable pitch H-type VAWT rotor}

In this thesis, the MLP-ANN structure is used for mapping of variable pitch angle H-type VAWT rotor and designing the intelligent pitch angle control system. The MLP-ANN structure is discussed in Section A2 in Appendix A. For each blade, a fully connected threelayer feedforward MLP-ANN with three types of input and one type of output is used for mapping the H-type VAWT rotor when the pitch angle is a variable. The number of nodes in the hidden is 20 . The features of the MLP-ANN are given TSRs $\left(1,1.7,2,2.5\right.$, and 3.3), $\theta\left(0^{\circ}\right.$ $\left.360^{\circ}\right)$, and the optimum pitch angles $\left(\beta_{\text {opt }}\right)$. However, maximum power coefficients $\left(C_{p, \max }\right)$ is chosen as the output data (target). These data sets are also divided into two parts: $75 \%$ for training and the remaining $25 \%$ for testing. The Levenberg-Marquardt back propagation algorithm (LM) is adopted as the training method because it can provide accurate predictions [81]. Sigmoid function is used as the activation function for neurons in the hidden layer. the training using LM algorithm ran for 1000 epochs of batch training.

\subsubsection{Using an MLP-ANN for the blade pitch angle control system}

The MLP-ANN method is also adopted for designing the intelligent control block depicted in Fig. 4.6 as the blade pitch angle controller for the H-type VAWT. The structure of the layered feedforward MLP-ANN for the control system is similar to that of the algorithm discussed in Section 4.2.1, but the training data differs with respect to input and output. Because a well-trained ANN with the correct structure is important for capturing the I/O relationship, the MLP-ANN model is trained based on more than 250,000 data points generated from the CFD calculations. The mean square error of the training process for the MLP-ANN model is shown in Fig. A5 in Appendix A. 


\subsection{Simulation results}

The H-Darrieus VAWT model is simulated using Matlab/Simulink to validate the proposed blade pitch angle control strategy in Fig. 4.6. The performance of the H-Darrieus VAWT in terms of power output is also evaluated in both cases: fixed and variable pitch angles.

\subsubsection{H-type VAWT mapping results}

Figure 4.7 (a) shows that LUT can estimate the power coefficients $\left(C_{p}\right)$ accurately at a uniform wind speed $\left(u_{\infty}=10 \mathrm{~m} / \mathrm{s}\right)$. The LUT is implemented using the exact CFD results with the azimuth angle range $\left(0^{\circ}-360^{\circ}\right)$ and optimum TSR $\left(\lambda_{o p t}=2.5\right)$ as inputs.
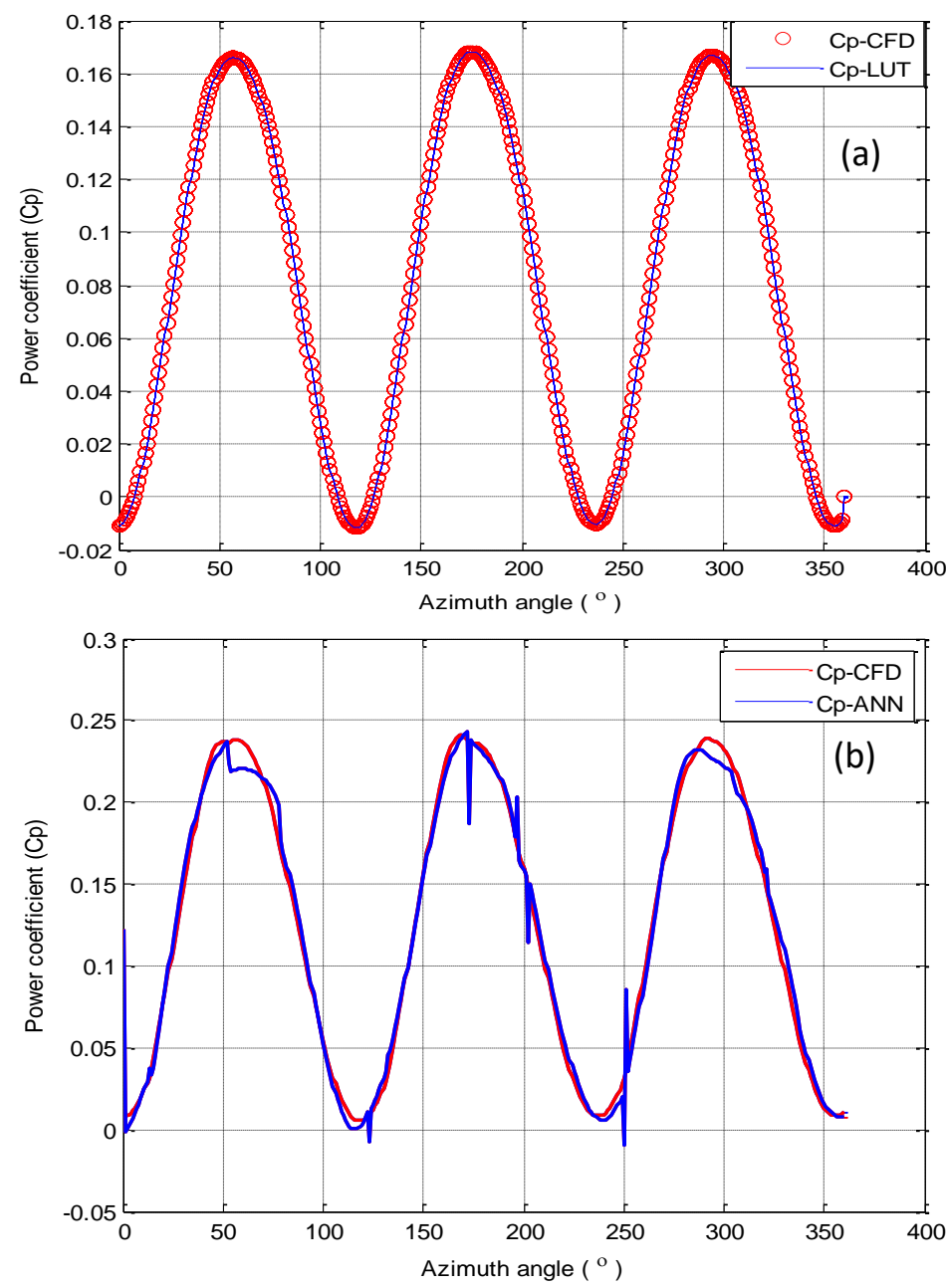

Figure 4.7. Mapping of the power coefficient $\left(C_{p}\right)$ at a wind speed of $10 \mathrm{~m} / \mathrm{s}$ by (a) the LUT for a fixed pitch angle VAWT and (b) the MLP-ANN for a variable pitch angle VAWT. 
As a result, there is no error between the value of $C_{p}$ obtained from the CFD model and the value of $C_{p}$ based on the LUT. In order to estimate the value of $C_{p}$ based on CFD results, the MLP-ANN algorithm is used for mapping the power coefficient for a variable pitch angle $\mathrm{H}$ type VAWT rotor in Matlab/Simulink model, as shown in Fig. 4.7 (b). In this case, the mean square error (MSE) is equal to $2.62 \times 10^{-4}$.

\subsubsection{Results for a pitch angle control system}

In this thesis, a Hybrid pitch angle control strategy based on combination of the proportional-integral-derivative (PID) and the MLP-ANN controllers is proposed to improve the power output quality at different operating regions, as shown in Fig. 4.6. The gains of the PID feedback pitch control system are tuned manually to be $K_{p}=0.00953, K_{i}=8.583 \times 10^{-5}$, and $K_{d}=0.00192$ for proportional, integral, and derivative, respectively. The performance of the proposed control system is evaluated for a uniform wind speed at a discrete time of $\mathrm{T}_{s}=2 \times 10^{-5} s$.

For each blade, Fig. 4.8 shows the reference and command pitch angle predicted by both the only MLP-ANN (i.e., 100\% MLP-ANN and 0\% PID) and the Hybrid proposed controller that used $10 \%, 20 \%, 50 \%$, and $80 \%$ of the PID pitch commands. It can be observed that, in general, both controllers can provide a good response for all reference values. However, the MLP-ANN controller and Hybrid controller with low PID command contributions (i.e., 10\% and $20 \%$ ) produce some overshoots, possibly due to outliers in the training data. These overshoots occurred for the second and third blades due to the blade wake effect in the upwind region. The rise time $\left(T_{r}\right)$, which is the time required for the signal to change from a given low value to a given high value, is used herein for measuring the performance of the control system. Table 4.1 shows the rise time values for both the Hybrid and MLP-NN pitch control systems. The mean square errors (MSEs) between the reference pitch angle and control pitch 
signals over three blades are listed in Table 4.2. The angle position error for each blade can be calculated by using MSE as follows:

$$
M S E=\frac{1}{n} \sum_{i=1}^{\mathrm{n}}\left(\beta_{\text {ref }, i}-\beta_{i}\right)^{2}
$$

where $n$ is the number of samples generated by the model simulation. Adding $10 \%$ and $20 \%$ of PID commands to the MLP-ANN controller, results in a shorter control rise time for the first blade while there are no significant differences for the second and third blades. Although the time is longer to reach the reference values of pitch angles for all blades when using the Hybrid controller with $80 \%$ and $50 \%$ of PID controller commands, MSE values are less than those for the MLP-ANN controller.

Table 4.1. Rise time for both the MLP-ANN and Hybrid pitch control systems at a uniform wind speed.

\begin{tabular}{cccccc}
\hline Rise time $\left(\boldsymbol{T}_{\boldsymbol{r}}, \mathbf{s e c}\right)$ & $\begin{array}{c}\text { MLP- } \\
\text { ANN }\end{array}$ & $\begin{array}{c}\text { Hybrid } \\
\text { 80\% PID }\end{array}$ & $\begin{array}{c}\text { Hybrid } \\
\mathbf{5 0 \%} \text { PID }\end{array}$ & $\begin{array}{c}\text { Hybrid } \\
\text { 20\% PID }\end{array}$ & $\begin{array}{c}\text { Hybrid } \\
\mathbf{1 0 \%} \text { PID }\end{array}$ \\
\hline Blade 1 & 0.1316 & 0.5909 & 0.3999 & 0.0600 & 0.0412 \\
Blade 2 & 0.1400 & 0.4500 & 0.1798 & 0.1563 & 0.1457 \\
Blade 3 & 0.0603 & 0.5510 & 0.0950 & 0.0719 & 0.0644 \\
\hline
\end{tabular}

Table 4.2. MSEs for both the MLP-ANN and Hybrid pitch control systems at a uniform wind speed.

\begin{tabular}{lccccc}
\hline (MSE) & MLP-ANN & $\begin{array}{c}\text { Hybrid } \\
\text { 80\% PID }\end{array}$ & $\begin{array}{c}\text { Hybrid } \\
\text { 50\% PID }\end{array}$ & $\begin{array}{c}\text { Hybrid } \\
\text { 20\% PID }\end{array}$ & $\begin{array}{c}\text { Hybrid } \\
\text { 10\% PID }\end{array}$ \\
\hline Blade 1 & 0.1256 & 0.0664 & 0.0284 & 0.0288 & 0.0156 \\
Blade 2 & 1.7904 & 0.3692 & 0.7819 & 1.4104 & 1.5592 \\
Blade 3 & 0.05 & 0.049 & 0.04 & 0.0539 & 0.0465 \\
\hline
\end{tabular}



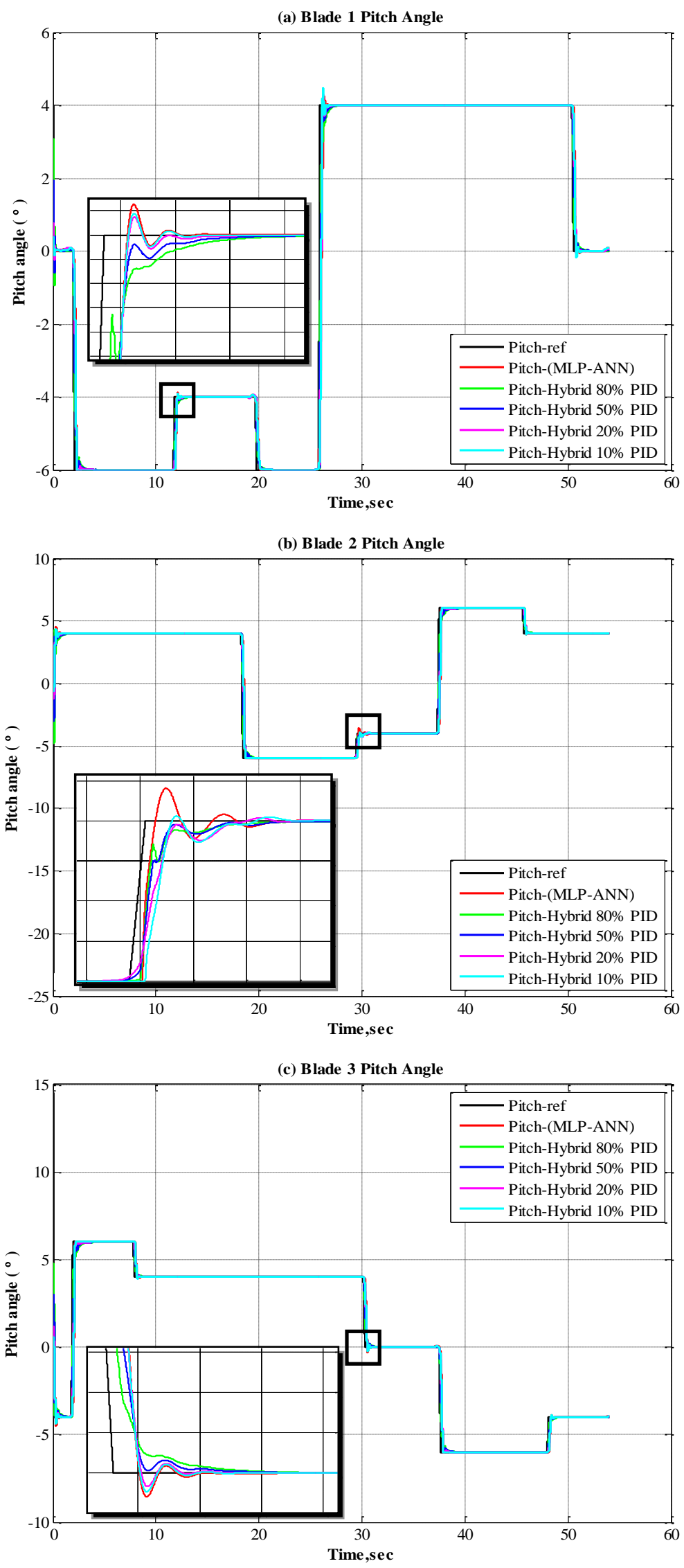

Figure 4.8. Response of the proposed pitch angle controller at $10 \mathrm{~m} / \mathrm{s}$. 
Effect of adding PID commands to MLP-ANN controller is observed in power output of an H-type VAWT. A comparison of power output achieved by the MLP-ANN and Hybrid controllers at a uniform wind speed is presented in Fig. 4.9, which reveals that the $P_{\text {ref }}$ curve and the power generation $\left(P_{g}\right)$ curves for all control scenarios are close to each other. The effect of variations in the pitch angle associated with both controllers, as shown in Fig. 4.8, can be clearly seen. Small discrepancies between the actual and reference power waveforms first occur during the second half of the simulation when the time is greater than approximately $37 s$. These discrepancies might be due to the large changes in the pitch angles for both the second (from $-4^{\circ}$ to $6^{\circ}$ ) and third (from $0^{\circ}$ to $-6^{\circ}$ ) blades that occur at the same time $(\approx 37.6 s)$. Although the discrepancies are reduced when the MLP-ANN control system is used, adding only a 10 percent of PID commands to MLP-ANN can reduce the MSE to $87 \%$, $12 \%$, and $7 \%$ for the first, second and third blade, respectively as shown in Table 4.2. An additional observation is that, for all curves, the peaks of the output power occur when the pitch angles of any two blades are positive. This finding means that the positive pitch angles decrease the angle of attack and, therefore, effect of flow separation is reduced accordingly.

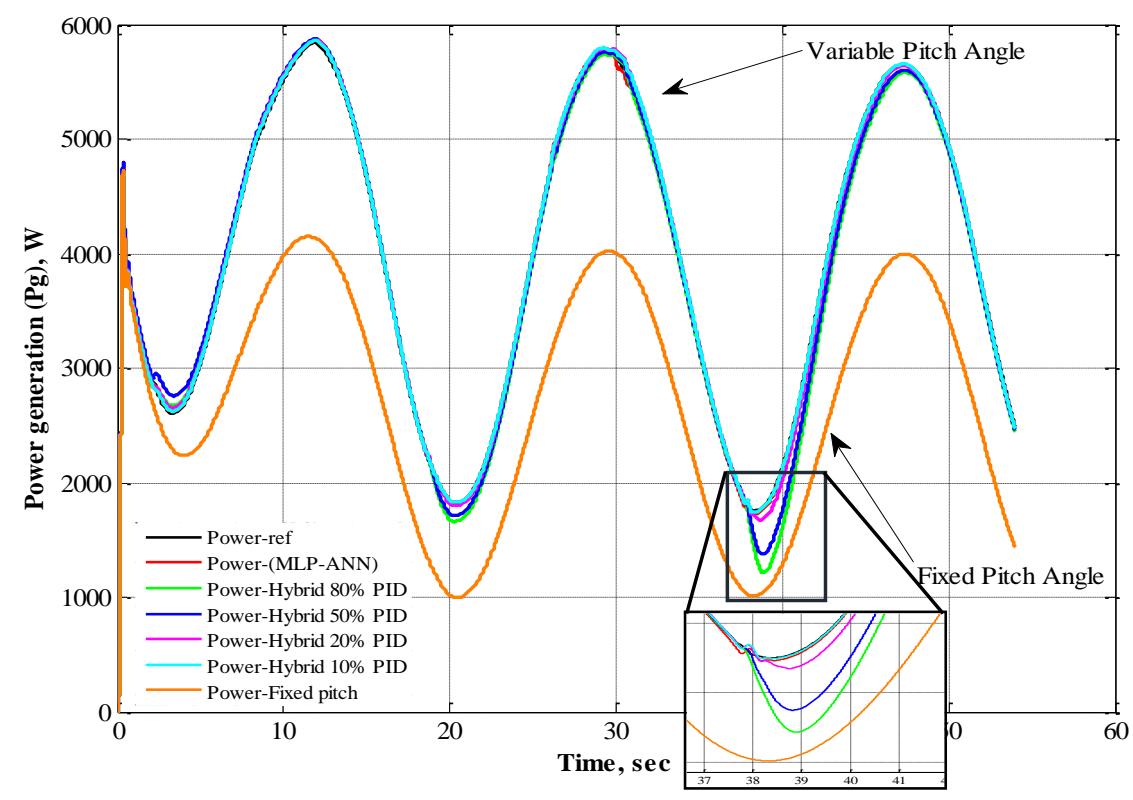

Figure 4.9. Power output from fixed and variable pitch angle H-type VAWT at uniform wind speed. 
The simulation results for both fixed and variable pitch H-type VAWTs are also shown in Fig. 4.9. Based on the individual blade pitching compared to fixed pitch for all blades, the performance of the H-type VAWT is improved in terms of power output. The H-type VAWT with the pitch control systems depicted in Fig. 4.6 can harness more wind energy under different operating conditions. In fact, compared to the fixed pitch angle H-type VAWT case, the power output is increased by around $25 \%$ for a uniform wind speed of $10 \mathrm{~m} / \mathrm{s}$.

The areas below all of the power curves in Fig. 4.9 represent the total gross energy harnessed by the H-type VAWT for fixed and variable pitch cases. Numerical integration using the trapezoidal rule is performed to calculate the total gross energy. The results for Hybrid controller (10\% of PID) are listed in Table 4.3.

Table 4.3.Wind energy prediction using fixed and variable pitch angle H-type VAWT models.

\begin{tabular}{ccc}
\hline Energy & Fixed Pitch $(\boldsymbol{E} \boldsymbol{f})$ & Var. Pitch $(\boldsymbol{E v})$ \\
\hline $\mathbf{k W h}$ & 0.0409 & 0.0594 \\
\hline
\end{tabular}

As well, for both the variable and fixed pitch angle configurations, the area between the power output curves represents the additional energy $\left(E_{a}\right)$ harnessed through pitching. It can be calculated as follows:

$$
E_{a}=E v-E f=0.0185 \mathrm{kWh}
$$

\subsubsection{Power output analysis}

In the variable pitch angle case, part of the total gross energy $(E v)$ is consumed within the plant due to the work needed to pitch the blades. These energy losses should be taken into account to calculate the net generation of energy $\left(E_{\text {net }}\right)$. $E_{\text {net }}$ can be calculated for the variable pitch angle case as follows:

$$
E_{\text {net }}=E v-E_{\text {loss }}
$$

The power losses $\left(P_{\text {loss }}\right)$ is calculated using the following formula 


$$
P_{\text {loss }}=P_{\text {servo }}+P_{c}
$$

where $P_{\text {servo }}$ is the power dissipated by the blade servomotors, and $P_{c}$ is the power loss due to the centrifugal force which acts on each blade when rotating. The required torque for the servomotor to rotate the blades is [82]:

$$
T_{s i}=I_{i} \times \ddot{\beta}_{i}, \quad i=1,2,3
$$

where $\beta$ is the pitch angle control signals $\left(-6^{\circ}\right.$ to $\left.6^{\circ}\right)$, and $I$ is the blade moment of inertia in $\mathrm{kg} \cdot \mathrm{m}^{2}$, which is calculated for each blade using Solidworks [83] $\left(I_{1}=I_{2}=I_{3}=\right.$ $0.0616 \mathrm{~kg} \cdot \mathrm{m}^{2}$ ). From Eq. (4.10), the servomotor power for each blade can be derived as:

$$
P_{s i}=T_{s i} \cdot \dot{\beta}_{i} ; \quad i=1,2,3
$$

Now, the required power by the servomotor $P_{\text {servo }}$ can be obtained as

$$
P_{\text {servo }}=\sum_{i=1}^{3} P_{i} ; \quad i=1,2,3
$$

The power loss by the centrifugal force effect $\left(P_{c}\right)$ using blade mass $\left(m_{\text {blade }}\right) 9 \mathrm{~kg}$, rotor radius $0.85 \mathrm{~m}$, TSR $(\lambda) 2.5$, and a uniform wind speed $\left(u_{\infty}\right)$ of $10 \mathrm{~m} / \mathrm{s}$, is given by [84]:

$$
P_{c}=m_{\text {blade }} \cdot R^{2} \cdot \omega_{r}^{3}=m_{\text {blade }} \cdot R^{2} \cdot\left(\frac{\lambda u_{\infty}}{R}\right)^{3}=m_{\text {blade }} \cdot \frac{\left(\lambda u_{\infty}\right)^{3}}{R}
$$

Table 4.4 shows the results of power analysis. It can be deduced that the power consumption by the control devices is only about $0.001 \mathrm{kWh}$ by using Eq. (4.13). In general, $E_{\text {loss }}$ are very small due to the small scale size of the H-type VAWT in terms of the weight of the blades and the small allowable range of pitch angle variations. Even with the power losses considered, the performance of the variable pitch angle H-type VAWT in terms of power output is still considerably better than the performance of the fixed pitch angle H-type VAWT.

Table 4.4. Net energy of the H-type VAWT model.

\begin{tabular}{ccc}
\hline $\boldsymbol{E} \boldsymbol{v}, \mathbf{k W h}$ & $\boldsymbol{E}_{\text {loss, } \mathbf{k W h}}$ & $\boldsymbol{E}_{\text {net }, \mathbf{k W h}}$ \\
\hline 0.0594 & $8.3096 \mathrm{e}-05$ & 0.0593 \\
\hline
\end{tabular}




\subsection{Conclusion}

Small- and medium-sized VAWTs can be utilized effectively as stand-alone wind energy generation sources if their efficiency can be further enhanced. In this chapter, an intelligent control algorithm based on neural network approach is proposed for designing an individual active blade pitch control system for an H-type VAWT as a means of improving its power generation performance. The CFD results in Chapter 3 have been utilized for designing control parameters for an H-type VAWT model using the MatlablSimulink. Because of the mathematical complexity associated with modeling the dynamic response of an H-type VAWT rotor, the CFD results have been applied for mapping the fixed and variable pitch angle system models of the H-type VAWT rotor using LUT as well as MLP-ANN approches. Also, a novel controller based MLP-ANN has been developed for controlling the blade pitch angle of a Darrieus H-type VAWT. A conventional controller (PID) is combined with an MLP-ANN controller (referred to as Hybrid controller) for controlling each blade individually. Although the ability to track the desired pitch angle is comparable for both controllers (i.e., MLP-ANN and hybrid), the improvement achieved when the small gains of PID commands are added has been clearly observed in the power output curves, where the effect of variations in the pitch angle associated with both controllers can be clearly seen. The results reveal that, compared to the fixed pitch angle operation, the blade pitching technique clearly increases the power output of the H-type VAWT, with a percentage improvement that exceeds $25 \%$. A rigorous mathematical stability analysis of the proposed pitch control system for an H-type VAWT will be presented in next chapter. 


\section{CHAPTER 5}

\section{STABILITY ANALYSIS ${ }^{3}$}

\subsection{Introduction}

A wind power generation system is a nonlinear time-varying system because of wind variations. Therefore, it is important to ensure the system's stability for the reliable and safe operation of the machine in all wind conditions [89]. Lyapunov theory is used widely for stability analysis of nonlinear systems. This chapter is composed of four sections including introduction. Section 2 begins with a mathematical description of an H-type VAWT. Section 3 includes the stability analysis of an H-type VAWT system. Analysis results based on Lyabunov Theory will be discussed in Section 4.

\subsection{H-type Vertical Axis Wind Turbine Modeling}

In general, there are two mathematical models for wind turbines are investigated by many studies [85][86][87][88]. The primary model is a variable speed (multi-input) and the secondary model which is a constant speed (single-input) second order model, the purpose of which is to simplify the nonlinear designs and to perform initial investigations with reduced complexity. The difference between a constant speed turbine and a variable speed turbine, is that the generator torque cannot be controlled. Therefore, the input data set of a variable speed turbine are the pitch angle and the generator torque, while the constant speed turbine model has the pitch angle as the only input [86]. This thesis uses a simple constant speed model that is a nonlinear second order system. The main components of an H-type VAWT are shown in Fig. 4.1 in chapter 4 . The wind turbine characteristics that will be considered in this chapter are:

- Aerodynamics

- Turbine mechanics

\footnotetext{
${ }^{3}$ Submitted in IEEE Transaction on Sustainable Energy, 2019
} 
- Generator dynamics

- Actuator dynamics

\subsubsection{Aerodynamic block}

The aerodynamic model of an H-type VAWT is discussed in Section 4.1.1. Eqs. (4.1) to (4.3) will be used in this chapter to conduct the stability analysis.

\subsubsection{Mechanical block:}

This block is represented in wind turbine models using 2-mass drive train model as shown in Fig. 5.1.

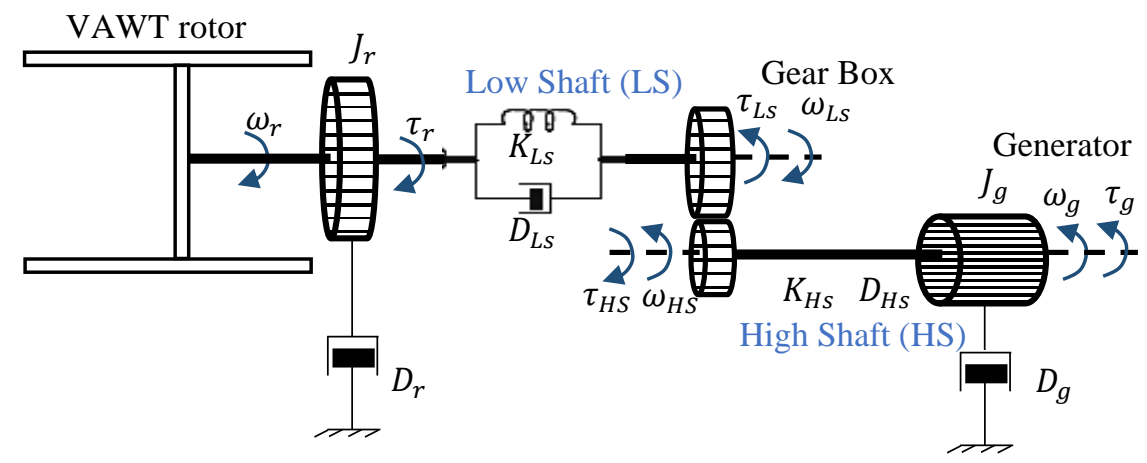

Figure 5.1. A wind turbine drive-train model based on a 2-mass model.

By using the free body diagram, the torque about a shaft can be developed as equation

$$
\tau=J \ddot{\theta}+D \dot{\theta}+K \theta
$$

where $\theta$ is the azimuth angle $\left(0^{\circ}-360^{\circ}\right), \dot{\theta}=\frac{\partial \theta}{\partial t}=\omega$ and $\ddot{\theta}=\frac{\partial \dot{\theta}}{\partial t}=\dot{\omega}$,

By applying Eq. (5.1) to all four rotating masses, the drive train can be modeled in the form as follows

$$
\begin{gathered}
J_{r} \ddot{\theta}_{r}+D_{L S}\left(\dot{\theta}_{r}-\dot{\theta}_{L S}\right)+K_{L S}\left(\theta_{r}-\theta_{L S}\right)+D_{r} \dot{\theta}_{r}=\tau_{r} \\
J_{g} \ddot{\theta}_{g}+D_{H S}\left(\dot{\theta}_{g}-\dot{\theta}_{H S}\right)+K_{H S}\left(\theta_{g}-\theta_{H S}\right)+D_{g} \dot{\theta}_{g}=-\tau_{g} \\
J_{r} \ddot{\theta}_{L S}-D_{L S}\left(\dot{\theta}_{r}-\dot{\theta}_{L S}\right)-K_{L S}\left(\theta_{r}-\theta_{L S}\right)=-\tau_{L S}
\end{gathered}
$$




$$
J_{g} \ddot{\theta}_{H S}-D_{H S}\left(\dot{\theta}_{g}-\dot{\theta}_{H S}\right)-K_{H S}\left(\theta_{g}-\theta_{H S}\right)=\tau_{H S}
$$

where $J_{r}, J_{g}$ are the moments of inertia of the wind turbine rotor and the generator [kg. $\left.m^{2}\right]$, respectively. $\tau_{r}, \tau_{g}$ are the rotor and generator torque $[\mathrm{N} \cdot \mathrm{m}]$, respectively. $\theta_{r}, \theta_{g}$ refer to the angular position of the rotor and the generator [rad], respectively. $\dot{\theta}_{r}, \dot{\theta}_{g}$ represent the wind turbine rotor and the generator speed $[\mathrm{rad} / \mathrm{s}]$, respectively. $\ddot{\theta}_{r}, \ddot{\theta}_{g}$ are their derivative. Similarly, all the above parameters are defined in terms of high and low shaft (HS and LS). Also, $D_{H S}, K_{L S}$ are the equivalent damping and stiffness for high and low shaft $[\mathrm{N} \cdot m \cdot s / \mathrm{rad}$, $[\mathrm{N} \cdot \mathrm{m} / \mathrm{rad}]$, respectively.

\section{Assumptions [86]-[88]}

1. $\ddot{\theta}_{L S}$ and $\ddot{\theta}_{H S}$ terms are equal to zero because of their direct coupling inside the gear box.

2. The rotor and generator self-damping $D_{r}$ and $D_{g}$ are neglected.

Rearranging equations (5.2) through (5.5) (i.e., Eq. (5.2) + Eq. (5.4) and Eq. (5.3) + Eq. (5.5)), it can be seen that there are two state equations.

$$
\begin{gathered}
J_{r} \ddot{\theta}_{r}=\tau_{r}-\tau_{L S} \\
J_{g} \ddot{\theta}_{g}=-\tau_{g}+\tau_{H S}
\end{gathered}
$$

But,

$$
\text { Gear ratio }=k=\frac{\tau_{L S}}{\tau_{H S}}=\frac{\ddot{\theta}_{g}}{\ddot{\theta}_{r}}
$$

Now, Eq. (5.6) and Eq. (5.7) can be simplified (Eq. (5.6) $+k^{*}$ Eq. (5.7)) as follows

$$
J_{r} \ddot{\theta}_{r}+k \cdot J_{g} \ddot{\theta}_{g}=\tau_{r}-k \cdot \tau_{g}-\tau_{L S}+\underbrace{k \cdot \tau_{H S}}_{\tau_{L S}}
$$

Then, dividing by $\ddot{\theta}_{r}$ :

$$
\begin{gathered}
\frac{J_{r} \ddot{\theta}_{r}}{\ddot{\theta}_{r}}+k \cdot J_{g} \underbrace{\ddot{\theta}_{g}}_{\underbrace{\ddot{\theta}_{g}}_{k}}=\frac{\tau_{r}-k \cdot \tau_{g}}{\ddot{\theta}_{r}} \\
\underbrace{J_{r}+k^{2} \cdot J_{g}}_{J_{\text {tot }}}=\frac{\tau_{r}-k \cdot \tau_{g}}{\dot{\omega}_{r}}
\end{gathered}
$$




$$
\dot{\omega}_{r}=\frac{\tau_{r}-k \cdot \tau_{g}}{J_{t o t}}
$$

The total equivalent moment of inertia of the drive system is

$$
J_{t o t}=J_{r}+k^{2} \cdot J_{g}
$$

\subsubsection{Pitch actuators:}

The dynamic model of pitch actuator can be described as a first-order transfer function [89]

$$
\dot{\beta}=\frac{1}{\tau_{\beta}} \beta_{c}-\frac{1}{\tau_{\beta}} \beta
$$

where $\beta$ and $\dot{\beta}$ are the pitch angles and their gradient, respectively, which are limited based on the time constant $\tau_{\beta}$. It can be assumed that $\beta_{c}$ is equal to $\beta$ because the dynamics of pitch systems operate much faster than that of the mechanical systems [50][90].

\subsubsection{Nonlinear state space representation}

The angular velocity of rotor $\omega_{r}$ is chosen as the system state variable, and the pitch angle $\beta_{c}$ is the input control variable. The deviation of the rotor speed and the actual value is the output. The model of H-type VAWT system can be described using Eq. (5.12) and Eq. (5.14). The nonlinear affine model of the system is

$$
\left\{\begin{array}{c}
\dot{x}=f(x)+g(x) u \\
y=h(x)
\end{array}\right.
$$

Where $x$ and $u$ are the state and input vector, respectively;

$$
x=\left[\begin{array}{c}
\omega_{r} \\
\beta
\end{array}\right], \quad u=\beta_{c}, \quad g(x)=\left[\begin{array}{c}
0 \\
1 \\
t_{\beta}
\end{array}\right],
$$




$$
\begin{gathered}
f(x)=\left[\begin{array}{c}
\frac{\tilde{\tau}_{r}^{\text {from Eq.(3) }}-k \cdot \tau_{g}}{\tilde{\tau}_{\text {tot }}} \\
-\frac{1}{t_{\beta}} \beta
\end{array}\right]=\left[\begin{array}{c}
\frac{1}{2 \cdot \omega_{r} \cdot J_{t o t}} \rho A u_{\infty}^{3} C_{p}(\lambda, \beta)-\frac{k \cdot \tau_{g}}{J_{\text {tot }}} \\
-\frac{1}{t_{\beta}} \beta
\end{array}\right] \\
=\left[\begin{array}{c}
\frac{1}{2 \cdot x_{1} \cdot J_{t o t}} \rho A u_{\infty}^{3} C_{p}(\lambda, \beta)-\frac{k \cdot \tau_{g}}{J_{\text {tot }}} \\
-\frac{1}{t_{\beta}} x_{2}
\end{array}\right]
\end{gathered}
$$

\subsection{Stability analysis of H-type VAWT system}

Stability of equilibrium points is usually characterized in the sense of Lyapunov (a Russian mathematician). More details about the Lyapunov Theory are provided in B1 in Appendix B.

In this thesis, stability of equilibrium points of the closed-loop system Eq. (5.15) is addressed for a variable pitch angle H-type VAWT by using Lyapunov stability theory. Although control of blade pitch simulation is a discrete-time problem, the stability analyses is simplified by assuming that the pitch control is continuous time. This simplification is valid because the control time step is much smaller than the turbine mechanical time constant, which depends on TSRs and then wind speed [88]. In the next Section 5.3.1, the equilibria points for the closed-loop system with constant pitch angle $\left(\beta=0^{\circ}\right)$ will be discussed. Then, the stability for variable pitch angle case will be analyzed.

\subsubsection{Constant pitch angle model}

For HAWTs, the stability of the system was examined in [85], [87], [88]. These references used a standard control strategy for generator torque control as follows:

$$
k \cdot \tau_{g}=k_{t} \omega_{r}^{2}, \quad k_{t}>0
$$

The control gain parameter $k_{t}$ is given by: 


$$
k_{t}=\frac{1}{2} \rho A R^{3} \frac{C_{p}}{\lambda^{3}}
$$

For optimum case (i.e., at the maximum $C_{p}$ value);

$$
k_{t}=\frac{1}{2} \rho A R^{3} \frac{C_{p, o p t}}{\lambda_{o p t}^{3}}
$$

The references also proved that if the curve

$$
C_{p, \text { cubic }}=\left(2 K_{t} / \rho A R^{3}\right) \lambda^{3}
$$

intersects the $C_{p}-\lambda$ curve at points $\lambda_{1}$ and $\lambda_{2}$, as shown in Fig. 5.2, then the equilibrium point $\left(C_{p, e q}, \lambda_{2}\right)$, where $C_{p, e q}=\left(2 k_{t} / \rho A R^{3}\right) \lambda_{2}^{3}$, is asymptotically stable with a region of attraction $\lambda \in\left(\lambda_{1}, \infty\right)$. However, $k_{t}$ is used in specific range to guarantee the stability of equilibrium $\left(C_{p, e q}, \lambda_{2}\right)$. Figure 5.2 also shows the upper bound of $k_{t}$ occurs when Eq. (5.4) is tangential to the $C_{p}-\lambda$ curve. By characterizing this limiting equilibrium point, the equilibrium $\left(C_{p, e q}, \lambda_{2}\right)$ is stable if the slope of the $\mathrm{C}_{\mathrm{p}}-\lambda$ curve at that point satisfies [87] as follows

$$
\left.\frac{\partial C_{p}}{\partial \lambda}\right|_{C_{p, e q}, \lambda_{2}}<\frac{3 C_{p, e q}}{\lambda_{2}}
$$

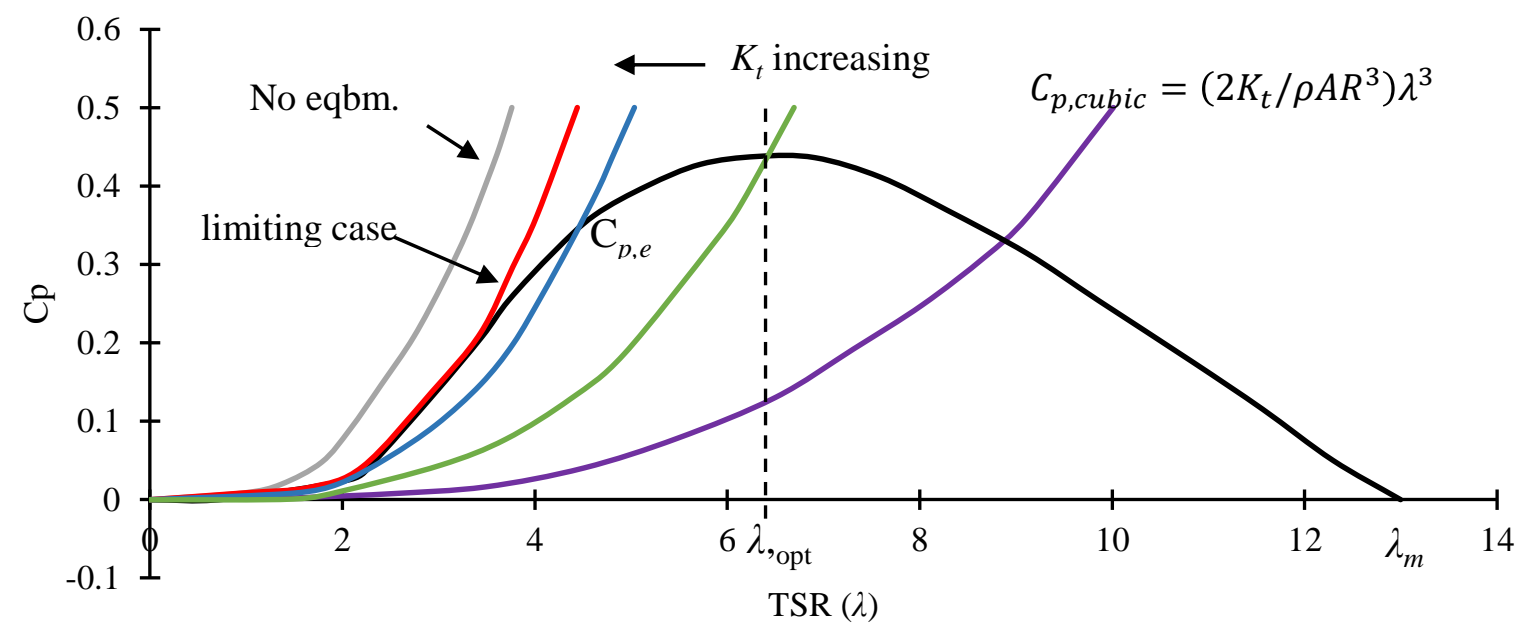

Figure 5.2. $\mathrm{C}_{\mathrm{p}}-\lambda$ curve and equilibrium points of a wind turbine. 


\subsubsection{Variable pitch angle model}

The primary control objective in this thesis is to maximize the power output using blade pitching. The torque control law is proposed as follows [85], [87], [88]:

$$
\tau_{g}=P_{r e f} / k \omega_{r}
$$

Where $P_{\text {ref }}$ is a constant rated power. From Eq. (5.12), the closed-loop equation is

$$
J_{t o t} \dot{\omega}_{r}=\tau_{r}-\frac{P_{r e f}}{\omega_{r}}
$$

For constant wind speed operation, and using Eq. (1.1),

$$
\dot{\lambda}=\frac{\dot{\omega}_{r} R}{u_{\infty}} \Rightarrow \dot{\omega}_{r}=\frac{\dot{\lambda} u_{\infty}}{\mathrm{R}}
$$

And from Eq. (4.3), $\tau_{r}=\frac{P_{m}}{\omega_{r}}$;

$$
J_{t o t} \frac{\dot{\lambda} u_{\infty}}{\mathrm{R}}=\frac{P_{m}}{\omega_{r}}-\frac{P_{r e f}}{\omega_{r}}
$$

This gives

$$
\dot{\lambda}=\frac{\mathrm{R} u_{\infty}^{2}}{J_{t o t} \omega_{r}}\left[\frac{1}{2} \rho A C_{p}(\lambda, \beta)-\frac{P_{r e f}}{u_{\infty}^{3}}\right]
$$

\section{Assumptions:}

In order to examine stability of operation for variable pitch angle H-type VAWT, it is assumed that the equilibria satisfies [87]
(1) $\frac{\partial C_{p}}{\partial \lambda}<0$ and
(2) $\frac{\partial c_{p}}{\partial \beta}<0$ for $\beta \geq \beta_{0}, \beta_{0}=0^{\circ}$ 
For each blade, the nonlinear pitch control (Hybrid controller) law for the H-type VAWT is given by

$$
\beta=\beta_{\left(M L P_{A N N}\right)}+\beta_{P I D}
$$

The following assumptions are made to describe the control law mathematically for stability analysis of proposed the H-type VAWT:

1. The error $e$ for both controllers PID and MLP-ANN is similar.

$$
\operatorname{error}(e)=\omega_{r, r e f}^{2}-\omega_{r}^{2},
$$

2. The "linear" activation equation is used instead of logistic "sigmoid" activation equation, which used in Chapter 4.

$$
\beta_{M L P-A N N}=e \sum_{i=1}^{N} w_{i, I n} w_{i, \text { Out }} ; \quad N=1,2
$$

Also, the PID control law is given by:

$$
\beta_{P I D}=k_{p}\left(\omega_{r, r e f}^{2}-\omega_{r}^{2}\right)+k_{d} \frac{\partial}{\partial t}\left(\omega_{r, r e f}^{2}-\omega_{r}^{2}\right)+k_{i} \int_{t_{0}}^{t}\left(\omega_{r, r e f}^{2}-\omega_{r}^{2}\right) \partial t
$$

3. The linear control will be simplified to PI controller because the derivative of the error in Eq. (5.24) is very small.

$$
\beta_{P I D}=\beta_{P I}=k_{p}\left(\omega_{r, r e f}^{2}-\omega_{r}^{2}\right)+k_{i} \int_{t_{0}}^{t}\left(\omega_{r, r e f}^{2}-\omega_{r}^{2}\right) \partial t
$$

By substituting Eq. (5.25) and Eq. (5.27) in Eq. (5.23) the pitch control law for each blade becomes

$\beta=e \sum_{i=1}^{N} w_{i, I n} w_{i, \text { out }}+\left[k_{p}\left(\omega_{r, r e f}^{2}-\omega_{r}^{2}\right)+k_{i} \int_{t_{0}}^{t}\left(\omega_{r, r e f}^{2}-\omega_{r}^{2}\right) \partial t\right]$

For any two steady wind speeds $u_{\infty, 1}$ and $u_{\infty, 2}$ the resulting equilibrium points $\mathrm{A}$ and $\mathrm{B}$ occur at the intersections of the $C p(\lambda, \beta)$ curve with $C_{p, c u b i c}=\left(2 K_{t} / \rho A R^{3}\right) \lambda^{3}$, as shown in Fig. 5.3. 


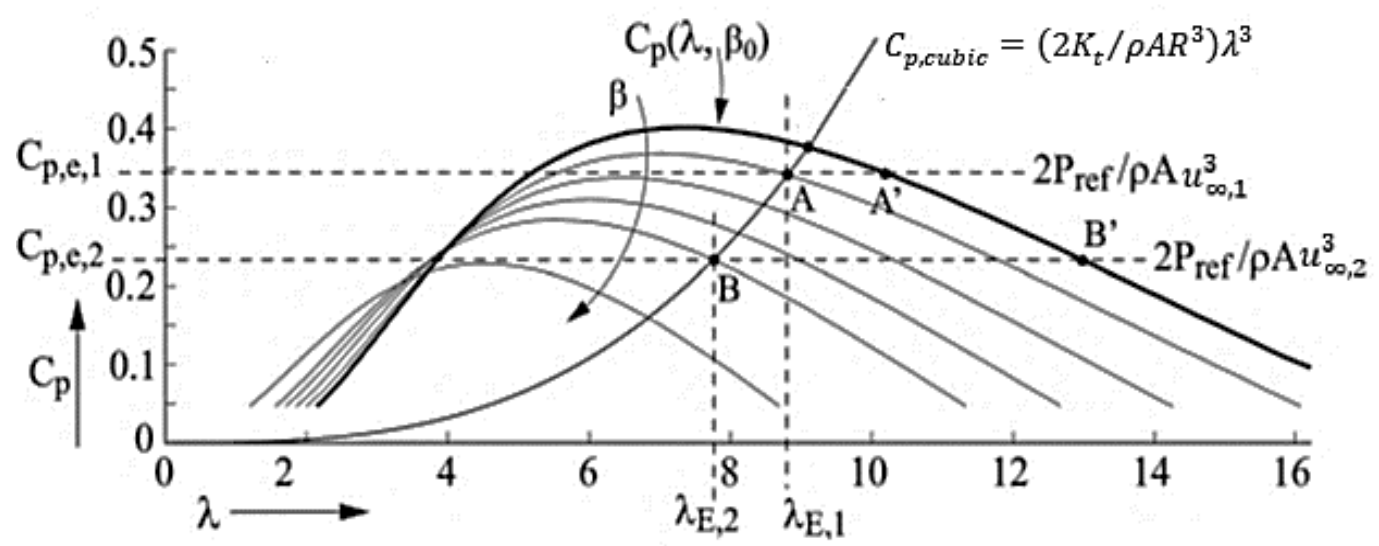

Figure 5.3. Equilibrium points for variable pitch angles wind turbine [87].

In order to analyze the stability of these equilibrium points [87], let

$$
\gamma=\frac{\lambda^{2}}{2} \Rightarrow \dot{\gamma}=\lambda \dot{\lambda}
$$

Substituting Eqs. (1.1) and (5.12) in Eq. (5.28), leads to

$$
\dot{\gamma}=\left(\frac{\omega_{r} R}{u_{\infty}}\right)\left(\frac{\mathrm{R} u_{\infty}^{2}}{J_{t o t} \omega_{r}}\left[\frac{1}{2} \rho A C_{p}(\lambda, \beta)-\frac{P_{r e f}}{u_{\infty}^{3}}\right]\right) ; \Rightarrow \dot{\gamma}=K L
$$

Where;

$$
K=\left(\frac{u_{\infty} R^{2}}{J_{\text {tot }}}\right) ; L=\left(\left[\frac{1}{2} \rho A C_{p}(\lambda, \beta)-\frac{P_{r e f}}{u_{\infty}^{3}}\right]\right)
$$

Defining the dynamics of the error variable $\tilde{\gamma}$ as follows [87], [88]:

$$
\tilde{\gamma}=\gamma-\gamma_{e q}
$$

Where $\gamma_{e q}=\frac{\lambda_{e q}^{2}}{2}$ and $\lambda_{e q}$ could be equal to either $\lambda_{E, 1}$ or $\lambda_{E, 2}$.

$$
\tilde{\gamma}=\frac{\lambda^{2}}{2}-\frac{\lambda_{e q}^{2}}{2} \Rightarrow \dot{\tilde{\gamma}}=\lambda \dot{\lambda}=K L
$$

So, the stability of the equilibrium $\tilde{\gamma}=0$ should be investigated. The error in Eq. (5.24) will be described at the equilibrium case as follows;

$$
\operatorname{error}(e)=\omega_{r}^{2}-\omega_{r, e q}^{2},
$$

By substituting in Eq. (5.28), leads to

$$
\beta=\left(\omega_{r}^{2}-\omega_{r, e q}^{2}\right) \overbrace{\sum_{i=1}^{N} w_{i, I n} w_{i, \text { out }}}^{m}+\left[k_{p}\left(\omega_{r}^{2}-\omega_{r, e q}^{2}\right)+k_{i} \int_{t_{0}}^{t}\left(\omega_{r}^{2}-\omega_{r, e q}^{2}\right) \partial t\right]
$$


Where $m$ is sum of weights. Taking the derivative of $\beta$

$$
\dot{\beta}=2 m \omega_{r} \dot{\omega}_{r}+2 k_{p} \omega_{r} \dot{\omega}_{r}+k_{i}\left(\omega_{r}^{2}-\omega_{r, e q}^{2}\right)
$$

But

$$
\omega_{r}=\frac{\lambda u_{\infty}}{R} \Rightarrow \dot{\omega}_{r}=\frac{\dot{\lambda} u_{\infty}}{R}, \text { and } \omega_{r, e q}=\frac{\lambda u_{e q} u_{\infty}}{R}
$$

Then

$$
\dot{\beta}=2 m\left(\frac{u_{\infty}}{R}\right)^{2} \lambda \dot{\lambda}+2 k_{p}\left(\frac{u_{\infty}}{R}\right)^{2} \lambda \dot{\lambda}+2 k_{i}\left(\frac{u_{\infty}}{R}\right)^{2}\left(\frac{\lambda^{2}}{2}-\frac{\lambda_{e q}^{2}}{2}\right)
$$

Substituting $(\lambda \dot{\lambda})$ from Eq. (5.32),

$$
\dot{\beta}=2 m\left(\frac{u_{\infty}}{R}\right)^{2} \dot{\tilde{\gamma}}+2 k_{p}\left(\frac{u_{\infty}}{R}\right)^{2} \dot{\tilde{\gamma}}+2 k_{i}\left(\frac{u_{\infty}}{R}\right)^{2} \tilde{\gamma}
$$

Now, taking the derivative of Eq. (5.32):

$$
\begin{gathered}
\ddot{\tilde{\gamma}}=K \frac{\partial L}{\partial \lambda} \dot{\lambda}+K \frac{\partial L}{\partial \beta} \dot{\beta}=K \frac{\partial L}{\partial \lambda} \frac{\dot{\tilde{\gamma}}}{\lambda}+K \frac{\partial L}{\partial \beta}\left(2 m\left(\frac{u_{\infty}}{R}\right)^{2} \dot{\tilde{\gamma}}+2 k_{p}\left(\frac{u_{\infty}}{R}\right)^{2} \dot{\tilde{\gamma}}+2 k_{i}\left(\frac{u_{\infty}}{R}\right)^{2} \tilde{\gamma}\right) \\
\ddot{\tilde{\gamma}}=\left(K \frac{\partial L}{\partial \lambda} \frac{1}{\lambda}+K \frac{\partial L}{\partial \beta}\left(2 m\left(\frac{u_{\infty}}{R}\right)^{2}+2 k_{p}\left(\frac{u_{\infty}}{R}\right)^{2}\right)\right) \dot{\tilde{\gamma}}+2 k_{i} K \frac{\partial L}{\partial \beta}\left(\frac{u_{\infty}}{R}\right)^{2} \tilde{\gamma} \\
\Rightarrow \ddot{\tilde{\gamma}}+f(\lambda, \beta) \dot{\tilde{\gamma}}+g(\lambda, \beta) \tilde{\gamma}=0
\end{gathered}
$$

where;

$$
f(\lambda, \beta)=-\left(K \frac{\partial L}{\partial \lambda} \frac{1}{\lambda}+K \frac{\partial L}{\partial \beta}\left(2 m\left(\frac{u_{\infty}}{R}\right)^{2}+2 k_{p}\left(\frac{u_{\infty}}{R}\right)^{2}\right)\right), g(\lambda, \beta)=-2 k_{i} K \frac{\partial L}{\partial \beta}\left(\frac{u_{\infty}}{R}\right)^{2}
$$

From Eqs. (5.29) and (5.37) and the assumptions 1 and 2 in Section 5.3.2, the nonlinear model for a variable pitch angle H-type VAWT system can be represented as follows:

$$
\begin{gathered}
f(\lambda, \beta)=-\left(K \frac{\partial C_{p}(\lambda, \beta)}{\partial \lambda} \frac{1}{\lambda}+K \frac{\partial C_{p}(\lambda, \beta)}{\partial \beta}\left(2 m\left(\frac{u_{\infty}}{R}\right)^{2}+2 k_{p}\left(\frac{u_{\infty}}{R}\right)^{2}\right)\right)>0 ; \\
g(\lambda, \beta)=-2 k_{i} K \frac{\partial C_{p}(\lambda, \beta)}{\partial \beta}\left(\frac{u_{\infty}}{R}\right)^{2}>0
\end{gathered}
$$


Now, the Lyapunov function candidate (the energy-like function) can be defined as [87], [91], [92]

$$
V=\frac{1}{2} \dot{\tilde{\gamma}}^{2}+\int_{0}^{\widetilde{\gamma}} g(\lambda, \beta) \tilde{\gamma} d \tilde{\gamma}
$$

Note that $V$ is positive definite. The derivative of $V$ along the system trajectories of Eq. (5.37), is:

$$
\dot{V}=\dot{\tilde{\gamma}} \ddot{\tilde{\gamma}}+g(\lambda, \beta) \tilde{\gamma} \dot{\tilde{\gamma}}=-f(\lambda, \beta) \dot{\tilde{\gamma}}^{2} \leq 0
$$

It can be seen that $\dot{V}$ is only semi-negative definite. Based on Theorem $i$ in $\mathrm{B} 1$ in Appendix B it can be concluded that $\tilde{\gamma}=0$ is locally stable at $\lambda_{e q}$. Although the stability of control law in Eq. (5.23) is proven in the sense of Lyapunov, the asymptotic stability for the origin of system still needs to be established. In order to satisfy the asymptotic stability condition, the LaSalle's Invariance Principle is examined. The theorem states that if there are no system trajectories that can stay forever at points where $\dot{V}=0$ except at the origin, then the origin is asymptotically stable [91].

Let $S=\{x \in D \mid \dot{V}(x)=0\}$, and suppose that no solution can stay forever in $S$, other than the trivial solution. Then, the origin is asymptotically stable. To characterize the set $\mathrm{S}$ for Eq. (5.39), note that

$$
\dot{V}=0 \Rightarrow-f(\lambda, \beta) \dot{\tilde{\gamma}}^{2}=0 \Rightarrow \dot{\tilde{\gamma}}=0 \Rightarrow \ddot{\tilde{\gamma}}=0 \Rightarrow \tilde{\gamma}=0 \text { (i. e. }, \lambda=\lambda_{e q} \text { ) }
$$

Therefore, the only solution that can stay in $S$ for all $t$ is the trivial solution. Thus, the origin $(\tilde{\gamma}=0, \dot{\tilde{\gamma}}=0)$ is asymptotically stable. 


\subsection{Stability analysis results}

The main parameters that are used for equilibrium points stability analysis for variable pitch angle H-type VAWT system using Lyapunov theory (theorem i) and quadratic Lyapunov equation are:

$$
J_{\text {tot }}=100 \mathrm{~kg} / \mathrm{m}^{2} ; \tau_{g}=20 \mathrm{Nm} ; k=15 ; t_{\beta}=0.15 \mathrm{sec}
$$

The rated power $P_{\text {ref }}$ and rotor speed $\omega_{\text {ref }}$ for the H-type VAWT are chosen as $300 \mathrm{~W}$ and $20 \mathrm{rad} / \mathrm{sec}$, respectively. The $C_{p}(\lambda, \beta)$ curves are available in polynomial form for all pitch angles (see Section B2 in Appendix B including the Matlab code for stability analysis).

\section{- Using Lyapunov theory for equilibrium points}

In order to determine the equilibrium points $\left(\lambda_{e q}, C_{p, e q}\right)$, the cubic function $C_{p, c u b i c}$ is calculated by using Eq. (5.19) and Eq. (5.17). The $C_{p, \text { cubic }}$ curve intersects with $C_{p}(\lambda, \beta)$ curves at different pitch angles to give two intersection points $A$ and $B$ as shown in Fig. (5.4). Figure 5.4 also shows that both intersection points $A(2.2,0.2891)$ and $B(2.16,0.2727)$ are only found at pitch angles $\beta=0^{\circ}$ and $\beta=4^{\circ}$, respectively. Based on the assumptions 1 and 2 in Sec. 5.3.2, Equation (5.39) is used to investigate the stability for both points $A$ and $B$ considering $A$ and $B$ are equilibrium points. Table 5.1 clearly shows that $\dot{V} \leq 0$ (i.e., seminegative definite) for point $A$ while $B$ is positive definite. As a result, $A$ are locally stable based on the Lyapunov theorem $i$ (see Appendix B). However, $B$ is not stable point. In addition, the stability analysis of the system at low TSRs of $\lambda \leq 1$ is also investigated. The results showed that $\dot{V} \leq 0$ at all pitch angles. Therefore, this region will be used in the experimental validation next chapter. 


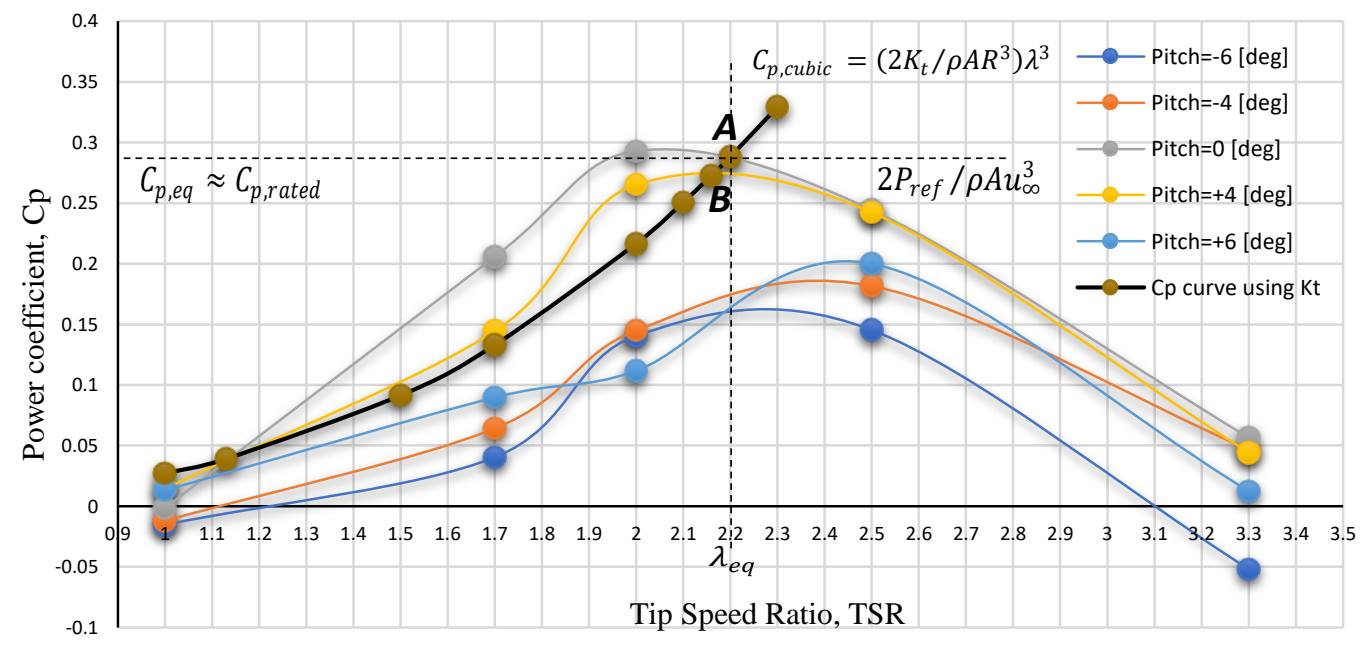

Figure 5.4. Equilibrium points for the H-type VAWT.

Table 5.1. Stability results using theorem $i$ for the H-type VAWT model

\begin{tabular}{|c|c|c|}
\hline TSR, $\lambda_{\text {eq }}$ & Pitch angle, $\beta\left[^{\circ}\right]$ & $\dot{V} \leq 0, E q .(5.39)$ \\
\hline 2.2 & 0 & -2.7490 \\
\hline 2.16 & 4 & 5.4259 \\
\hline
\end{tabular}

\section{- Using quadratic Lyapunov function}

To analyze the stability of an H-type VAWT, $x_{1}$ and $x_{2}$, which represent $\lambda$ and $\beta$ respectively, are obtained using the wind turbine model outlined in Eq. (5.15). For PID controller Eq. (5.26), the gains are selected as (see Section 4.3.2)

$k_{p}=0.00953 ; k_{d}=0.00192 ; k_{i}=8.583 \times 10^{-5}$;

For MLP-ANN controller, the control law is expressed as

$$
\beta_{M L P-A N N, l}=F(Z)=F\left(\sum_{i}^{N} w_{i, j} \cdot e_{i}\right) ; l=1,2,3
$$

where $F$ is the sigmoid function which is given by $F(Z)=1 /(1+\exp (-Z))$ and $e$ (error) is the input of MLP-ANN which is calculated as follows

$$
e=\left(\omega_{r}-\omega_{r, r e f}\right) \Rightarrow e=\left(\frac{x_{1} u_{\infty}}{R}-\omega_{r, r e f}\right)=\left(11.76 x_{1}-20\right) ;
$$




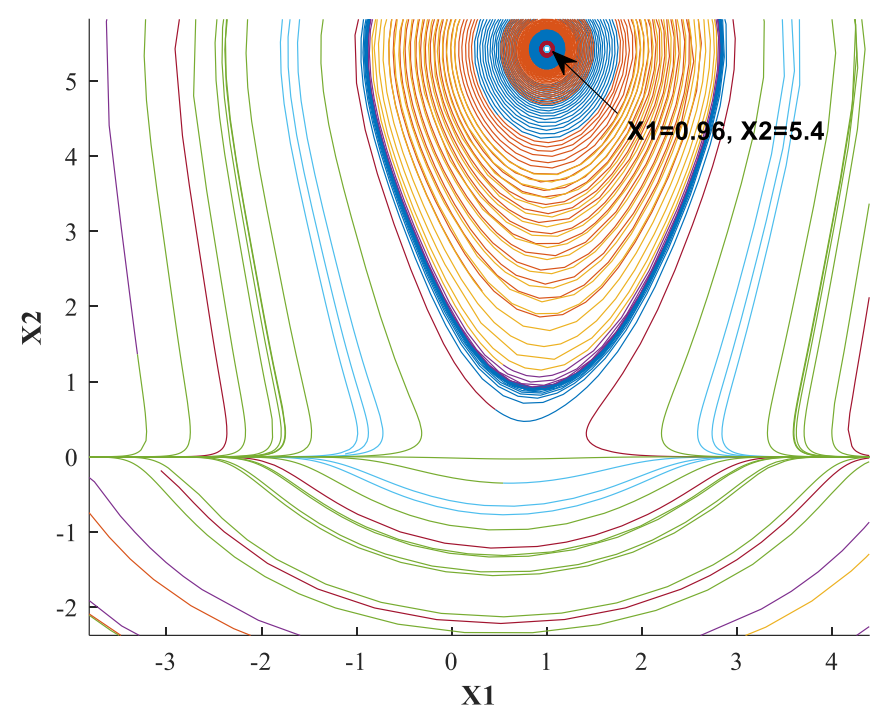

Figure 5.5. Phase diagram for the system Eq. (5.15).

Figure 5.5 shows the phase diagram for the system using Eq. (5.15). In order to investigate the stability for these equilibrium points, a change of variables is required to shift the equilibrium points to the origin. This produces

$$
s_{1}=x_{1}-0.96 s_{2}=x_{2}-5.41
$$

Then, evaluating the Jacobian matrix at $s=0$ (using linearization):

$$
A=\left.\frac{\partial f}{\partial x}\right|_{s=0}=\left[\begin{array}{ll}
\frac{\partial f_{1}}{\partial x_{1}} & \frac{\partial f_{1}}{\partial x_{2}} \\
\frac{\partial f_{2}}{\partial x_{1}} & \frac{\partial f_{2}}{\partial x_{2}}
\end{array}\right]=\left[\begin{array}{cc}
-0.6226 & 0.0206 \\
1.3780 & -6.6667
\end{array}\right]
$$

Matrix $A$ is called a stability matrix or a Hurwitz matrix. The eigenvalues and of Jacobian Matrix $A$ are used for vector field classification. The eigenvalues of $A$ are obtained as

$$
a_{1}=-0.6179, a_{2}=-6.6714
$$

It can be seen that all eigenvalues of $\mathrm{A}$ satisfy $R e a_{i}<0$, hence, the origin is asymptotically stable because $A$ is stable [91]. The stability can be informed based on the real part $(R e)$ of eigenvalues $(a)$ as illustrated in B3 in Appendix B. 


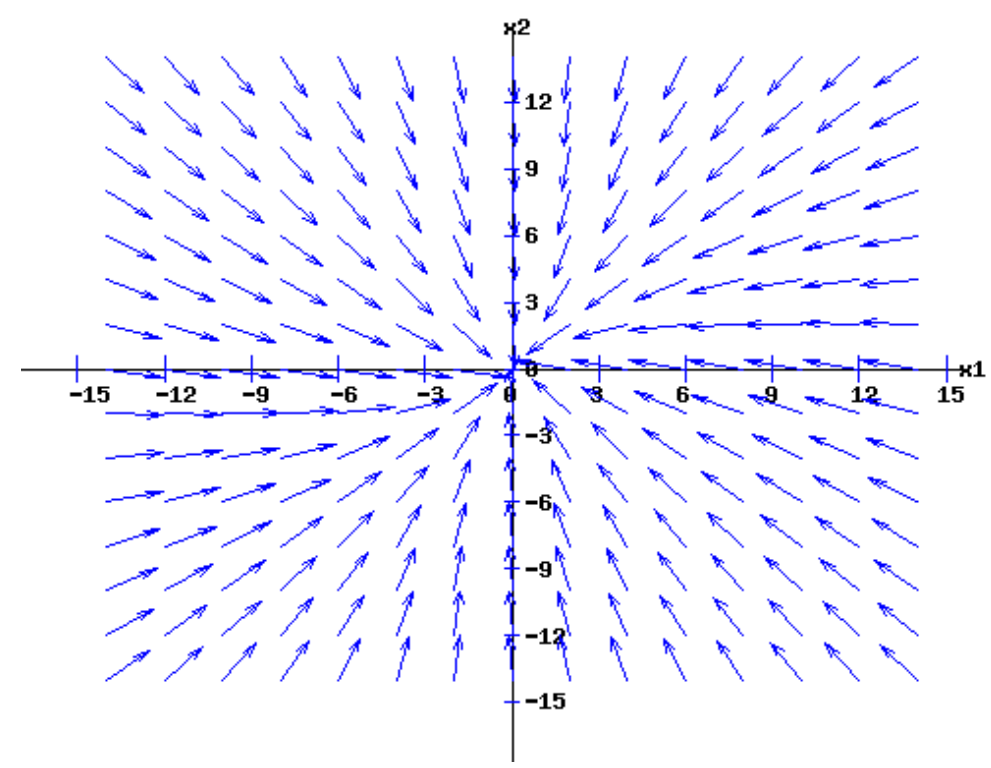

Figure 5.6. Phase portrait for matrix $A$.

In Fig. 5.6, the phase portrait for matrix $A$ shows that the origin is a stable node (attracting node).

Theorem iii [91] A matrix $A$ is a stability matrix, that is, $R e a_{i}<0$ for all eigenvalues of $A$, if and only if for any given definite symmetric matrix $Q$ there exists a positive definite symmetric matrix $P$ that satisfies the Lyapunov equation Eq. (B4) in Appendix B. Moreover, if $A$ is a stability matrix, then $P$ is the unique solution of Eq. (5.15).

In order to find $P$, let $Q=I$ (identity matrix) from Eq. (B4)

$$
A^{T} P+P A=-I
$$

So, the unique solution is

$$
P=\left[\begin{array}{cc}
0.8081 & 0.1531 \\
0.1531 & 0.1066
\end{array}\right]
$$

From matrix $P$, the eigenvalues of Lyapunov are 0.0747 and 0.08401 . Obviously, the system is positive definite and hence stable.

In order to extend the stability analysis of the H-type VAWT to different operating conditions shown in Fig. 3.3 in chapter 3, TSRs and pitch angles are assumed to be equilibrium points (i.e., $x_{1}$ and $x_{2}$ ). The stability is then analyzed by examining the existence of a matrix $P$ for all pitch angles considered. Results are listed in Table 5.2. 
Table 5.2. Matrix P status for all pitch angles.

\begin{tabular}{|c|c|c|c|c|c|c|c|c|c|c|c|c|c|c|c|}
\hline \multirow[b]{2}{*}{0} & \multicolumn{15}{|c|}{ Tip speed ratios $(\lambda)$} \\
\hline & & 1 & 1.1 & 1.2 & 1.3 & 1.5 & 1.7 & 1.9 & 2 & 2.1 & 2.2 & 2.3 & 2.5 & 3 & 3.3 \\
\hline (2) & -6 & $+v e$ & $+v e$ & $+v e$ & -ve & $-v e$ & $-v e$ & $-v e$ & $-v e$ & $-v e$ & $-v e$ & $+v e$ & $+v e$ & $+v e$ & $-v e$ \\
\hline 0 & -4 & $+v e$ & $+v e$ & $-v e$ & $-v e$ & -ve & -ve & $-v e$ & $-v e$ & $-v e$ & $+v e$ & $+v e$ & $+v e$ & $+v e$ & $-v e$ \\
\hline పี & $\mathbf{0}$ & $+v e$ & $-v e$ & $-v e$ & $-v e$ & $-v e$ & $-v e$ & $-v e$ & $-v e$ & $+v e$ & $+v e$ & $+v e$ & $+v e$ & $+v e$ & $-v e$ \\
\hline తే & 4 & $+v e$ & $+v e$ & $-v e$ & $-v e$ & $-v e$ & -ve & $-v e$ & -ve & $-v e$ & $+v e$ & $+v e$ & $+v e$ & $+v e$ & $-v e$ \\
\hline E & 6 & $+v e$ & $+v e$ & $+v e$ & $-v e$ & $-v e$ & $-v e$ & $-v e$ & $-v e$ & $-v e$ & $-v e$ & $+v e$ & $+v e$ & $+v e$ & $+v e$ \\
\hline
\end{tabular}

At low TSRs $(1<\mathrm{TSR}<1.1)$, a positive definite $P$ matrix $(+v e)$ exists for most pitch angles.

It means that the system is stable using pitch angle Hybrid controller in Eq. (5.28). This is important because a blade pitching is used to improve the self-stating capability of H-type VAWT at low TSRs. However, for $1.2<\mathrm{TSR}<2.1$, the system is unstable because of the negative definite $P$ matrix (-ve). The optimum power coefficients for all pitch angles occurs for $\mathrm{TSR} \geq 2$ as shown in Fig. 5.7. Table 5.2 also shows that the system is stable for 2.2 $<$ TSR $<3.3$. This will be useful for choosing the optimum operating point to maximize the system power output. The gray areas in Fig. 5.7 represent the stable domains of the H-type VAWT system.

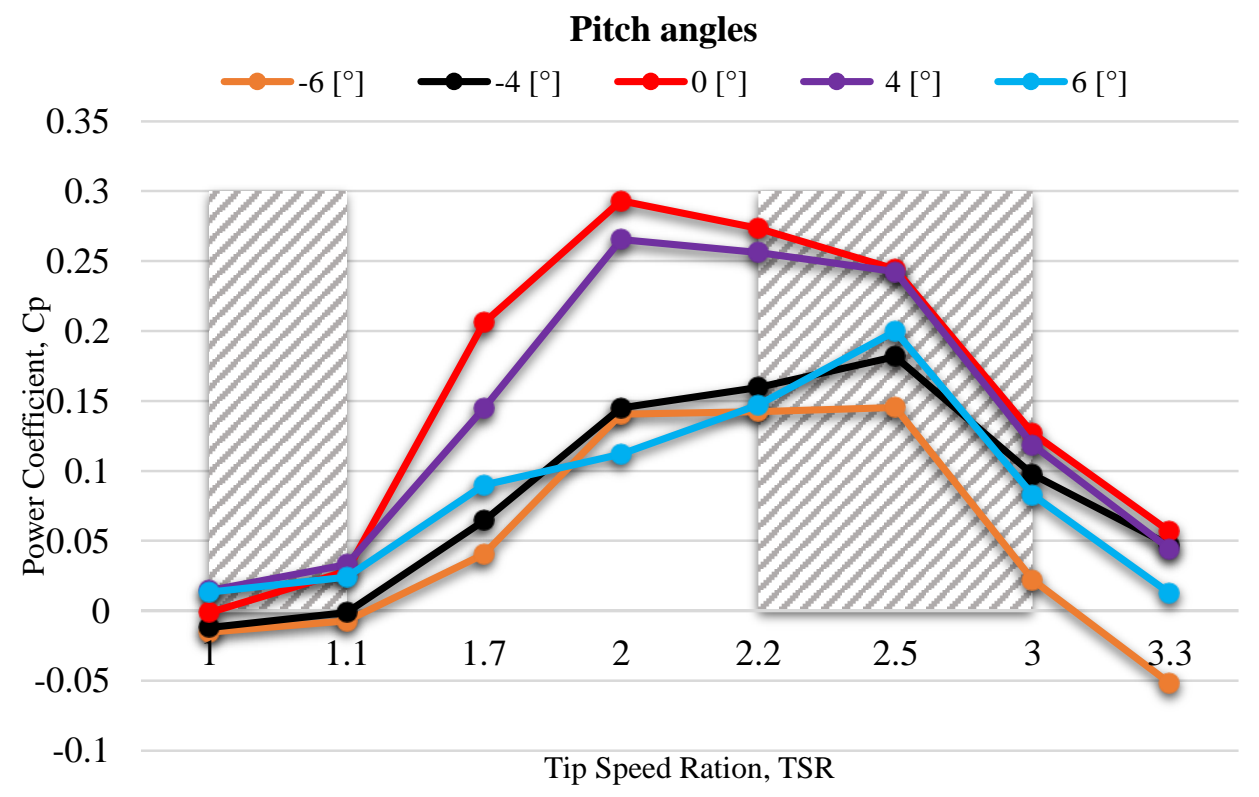

Figure 5.7. Stability domains for $C_{p}-\lambda$ curves at different pitch angles. 


\subsection{Conclusion}

In this chapter, the stability of the H-type VAWT has been investigated both fixed and variable pitch angle configurations. An H-type VAWT is modeled in nonlinear state space by determining the mathematical models for H-type VAWT components along with Hybrid control scheme. Stability was studied using the Lyapunov method and considering the turbine model and a Hybrid controller (i.e., PID and MLP-ANN controller). The system performance equations are linearized and expressed as simultaneous differential equations in state space and then analyzed using the Lyapunov direct method. Using Lyapunov's stability theorem, it was shown that system is asymptotically stable when the proposed blade pitch control law in Eq. (5.28) is applied to the H-type VAWT model in Eq. (5.15). 


\section{Chapter 6}

\section{AN H-TYPE VAWT EXPERIMENTAL SETUP: DESIGN AND VALIDATION ${ }^{4}$}

Some studies [9][48][49] investigated the effect of blade pitching on VAWT performance in terms of power generation and self-starting capability experimentally. However, these studies used simple mechanical mechanisms or simple controllers such as PID controller for blade pitching. In this thesis, the novel Hybrid controller is proposed to vary the blade pitch angles for an H-type VAWT. The effect of blade pitching on the H-type VAWT in terms of both power output and self-starting capability is numerically investigated, as discussed in Chapter 3 and Chapter 4. The results showed that the power output and self-starting capability are increased by $25 \%$ and $12 \%$, respectively. In order to validate these results experimentally, building an H-type VAWT with blade pitching capability to test the proposed controller in Chapter 4 is presented in this chapter.

\subsection{Design, Manufacturing \& Assembly an H-type VAWT}

The manufacturing and assembly phase will comprise five key stages: blade fabrication, blade hub and linkage manufacturing, main shaft and bearing setup, generator and main-shaft coupling, and finally the overall assembly.

\subsubsection{Modifications and blade fabrication}

Originally, the blade of an H-type VAWT was planned to be manufactured by using a 3D printing machine. However, due to the high cost of each piece (Nearly $\$ 2000$ per blade) this option was ruled out. To lower the cost of blade manufacturing and to gain better control over the shape of the blades, each blade is fabricated using three main components.

Two Aluminum round bars are placed through the three blade parts to align them straight as well as to act as pivot and fixed shaft around which variable pitching takes place. The

${ }^{4}$ Submitted in IEEE Transaction on Sustainable Energy, 2019 
round bar closer to the leading edge is $3 / 8$ inch (Fixed shaft-For pivoting) in diameter and the one closer to the trailing edge is 0.5 inches (Pitching shaft). The fixed shaft is also passed through two bearings that are placed inside the top and bottom parts to give the blade the rotation degree of freedom as shown in Fig. 6.1 (a) (bearing sketch is shown in Fig. C1 in Appendix C).

Based on the fabrication method selected, three 3D printed parts are initially placed onto the aluminum sheet and two Aluminum round bars are inserted into the bores of the interior parts, giving the structure shown in Fig. 6.1 (b). An Aluminum 5052 sheet with length of $1 \mathrm{~m}$, width of $0.51 \mathrm{~m}$ and thickness of $0.6-0.7 \mathrm{~mm}$ is wrapped around the 3 internal parts to give the blade (NACA0018 profile) its uniform and smooth shape. The three parts are then riveted on to the aluminum sheet at three locations for each part (optimized for maximum strength). The sheet is then bent by applying equal force at the top, middle and bottom regions. The bent sheet is now on top of the parts; the sheet is riveted again onto the parts from the new surface to complete the fabrication process as shown in Fig. 6.1 (c).
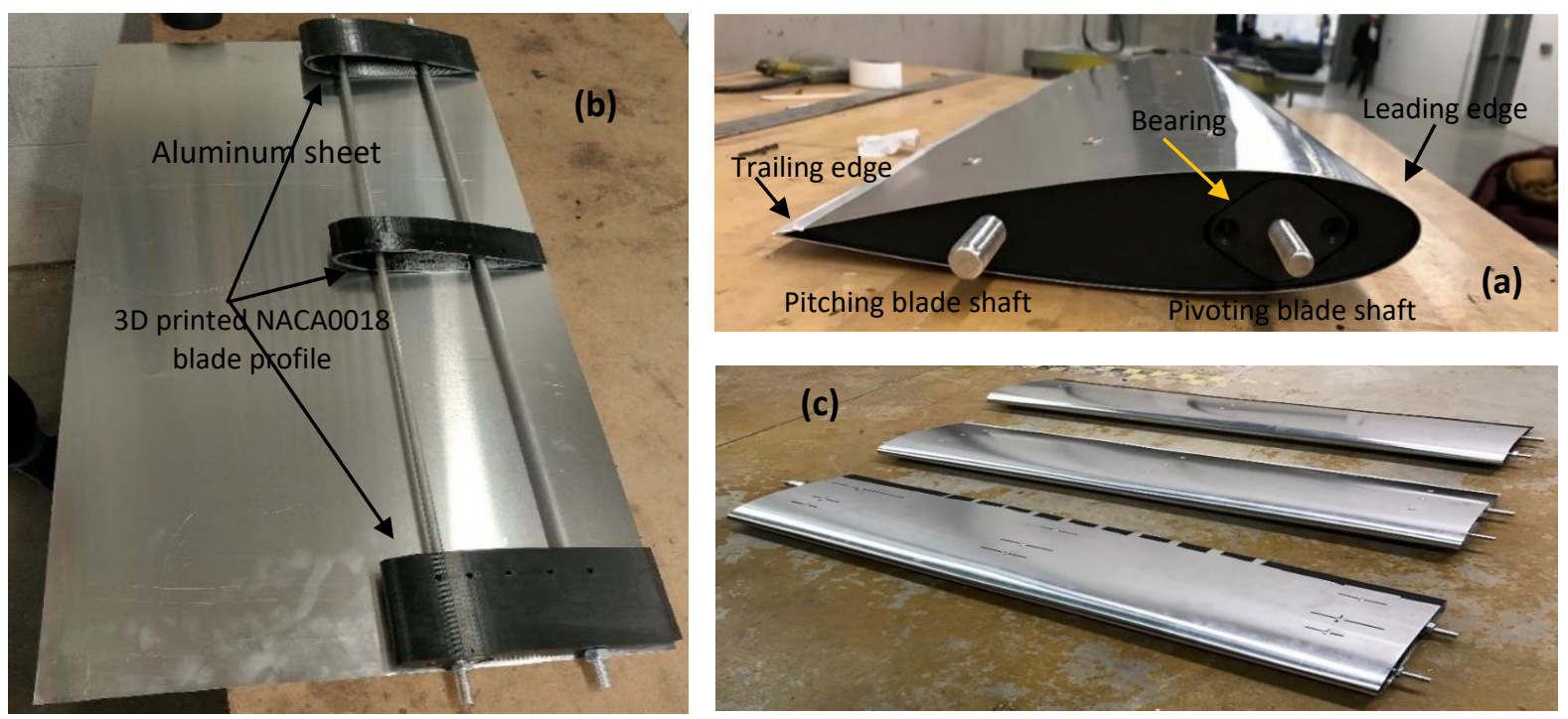

Figure 6.1. Fabricated NACA0018 Blade. 


\subsubsection{Blade hubs and linkages}

To connect the blades to the main shaft of rotor, star shaped linkages are used to allow the transfer of aerodynamic forces from the blades to the main shaft.

Minimizing deflection, machining cost, complexity, and weight are considered for designing the blade hubs and linkages. In this design, the hub is a circular disk with three pockets at $120^{\circ}$ apart for linkages to tightly inside them as shown in Fig. 6.2. The distance from the center of the main shaft to the center of the hole on the linkages is $0.85 \mathrm{~m}$. Therefore, the entire wind turbine is $1.7 \mathrm{~m}$ in diameter. Figure $\mathrm{C} 2$ in Appendix $\mathrm{C}$ shows a CAD design of blade hub and linkage. Figure 6.3 shows the expected deflection on the blade linkage that is around $3.5 \mathrm{~mm}$.

Two Aluminum tubes are welded on to a 0.75 -inch thick aluminum plate to form the hub for connecting the main shaft to the blades. Holes are made on the hub and the grooves set for the linkages to be slotted in.
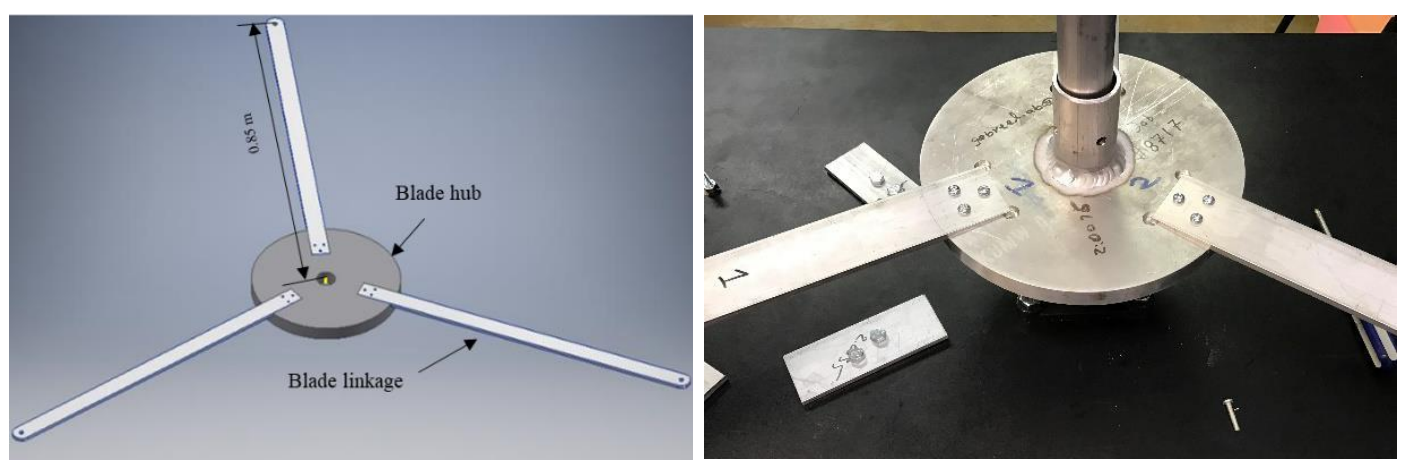

Figure 6.2. Blade hub and linkages.

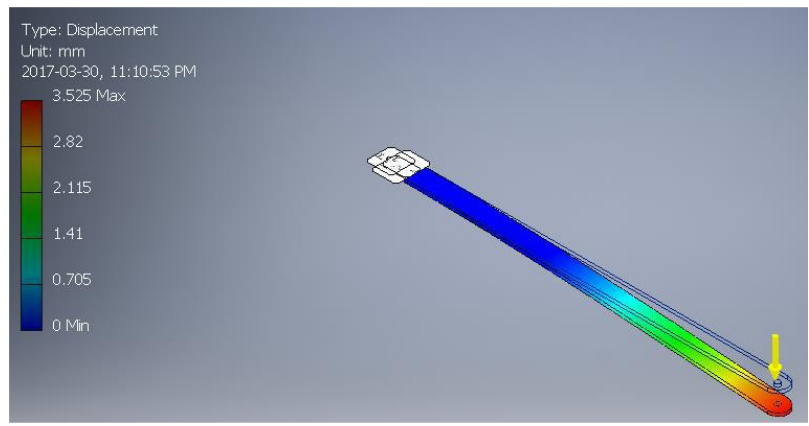

Figure 6.3. Deflection of the linkage. 


\subsubsection{The main shaft and its bearing}

The main shaft transmits the rotational energy to the generator through a transmission system. In the proposed design, the main shaft of the H-type VAWT is an Aluminum 6061 hollow shaft with wall thickness of $12.7 \mathrm{~mm}$, length of $1700 \mathrm{~mm}$ and diameter of $38.1 \mathrm{~mm}$ which exhibits some good mechanical properties, such as lightweight, great joining capability, comparatively high strength, and good machinability. Main shaft's bearing is the most important part in the system in terms of load bearing reliability. The selected bearing is attached to the base table to reduce the deflection that occurs by the weight of the rotating parts of the turbine. The entire wind turbine suspended above the base table weighs close to $30 \mathrm{~kg}$. However, during high RPM shaft rotation this weight is prone to magnification, which is called a dynamic radial load. Hence, with $30 \mathrm{~kg}$ static load the bearing should be able to withstand $75 \mathrm{~kg}$ (safety factor=2.5) of dynamic radial load. Figure 6.4 shows the selected bearing that have static radial load capacity of $2000 \mathrm{~kg}$ and maximum speed of $5800 \mathrm{RPM}$ based on the bearing specification shown in Fig. C3 in Appendix C.
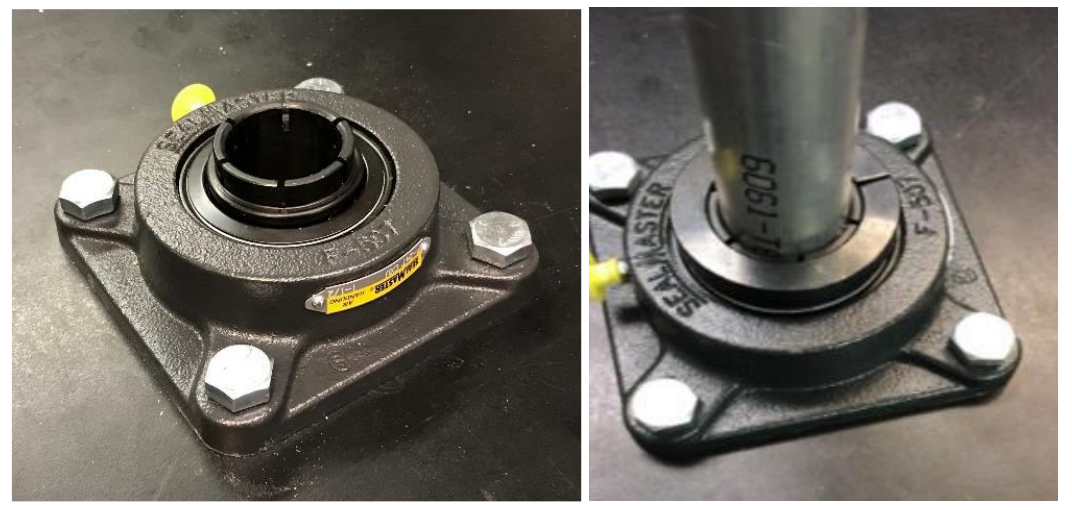

Figure 6.4. Main shaft's bearing installed on the table.

\subsubsection{Generator and Main Shaft Coupling}

This experimental setup uses a low RPM permanent magnet synchronous wind turbine generator (PMSG) that can produce 60 volts at only 100 RPM based on specification in Section C1 in Appendix C. The preliminary design encompasses a method of assembly that 
allows a direct contact between the shaft of the rotor and the shaft of the generator. For this direct linkage design to function perfectly, the turbine rotor should have an RPM close to the generator's angular velocity which is around 100 to $150 \mathrm{rpm}$. Yet, it is challenging for an $\mathrm{H}$ type VAWT rotor RPM reaches this angular velocity in real tests. Therefore, a transmission system is implemented to magnify the angular velocity of rotor to 100-150 RPM in order for the generator to produce power. The transmission system to couple the generator and main shaft is a pulley system. Figure 6.5 illustrates the pulley system setup under the base table. The larger pulley (11.75-inch diameter) is placed at the end of wind turbine's main shaft using a key and the smaller pulley (2-inch diameter) is attached to the shaft of the generator. The distance between the two pulleys is approximately 11 inches, which puts the 48 -inch belt in full tension to reduce torque losses. In order to lower the center of gravity of the wind turbine, the PMSG is placed under the base table which makes the turbine more stable and reduces the possibility of turbine tipping over when operating. As shown in Figure 6.5, the generator is mounted onto the welded steel cylinder that is attached on two of the table legs.

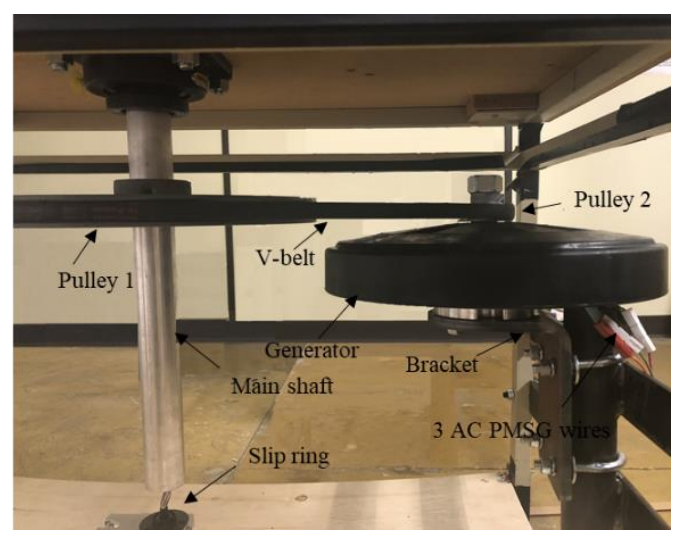

Figure 6.5. Pulley system for main shaft and generator coupling.

\subsubsection{Overall Assembly}

The mass requirement for each blade is specified at around 2-3 kilograms. Meeting this requirement means that the hubs and linkages will be able to withstand the load. However, there was noticeable deflection on the lower linkages due to weight of the blades causing 
extra vibrations and oscillations in the system. The deflection is accounted for in the initial design phase and specified at maximum of $4 \mathrm{~mm}$ allowed deflection. However, the bending on the linkages was approximately $55.56 \mathrm{~mm}$ based on the displacement-stress analysis as shown in Fig. C4 (a) in Appendix C. Therefore, a support structure was designed and manufactured to lift the linkages which reduced the bending to $1.345 \mathrm{~mm}$ as shown in Fig. C4 (b) in Appendix C. The proposed linkage support is shown in Fig. C5 in Appendix C. The final H-type VAWT assembly is shown in Fig 6.6.

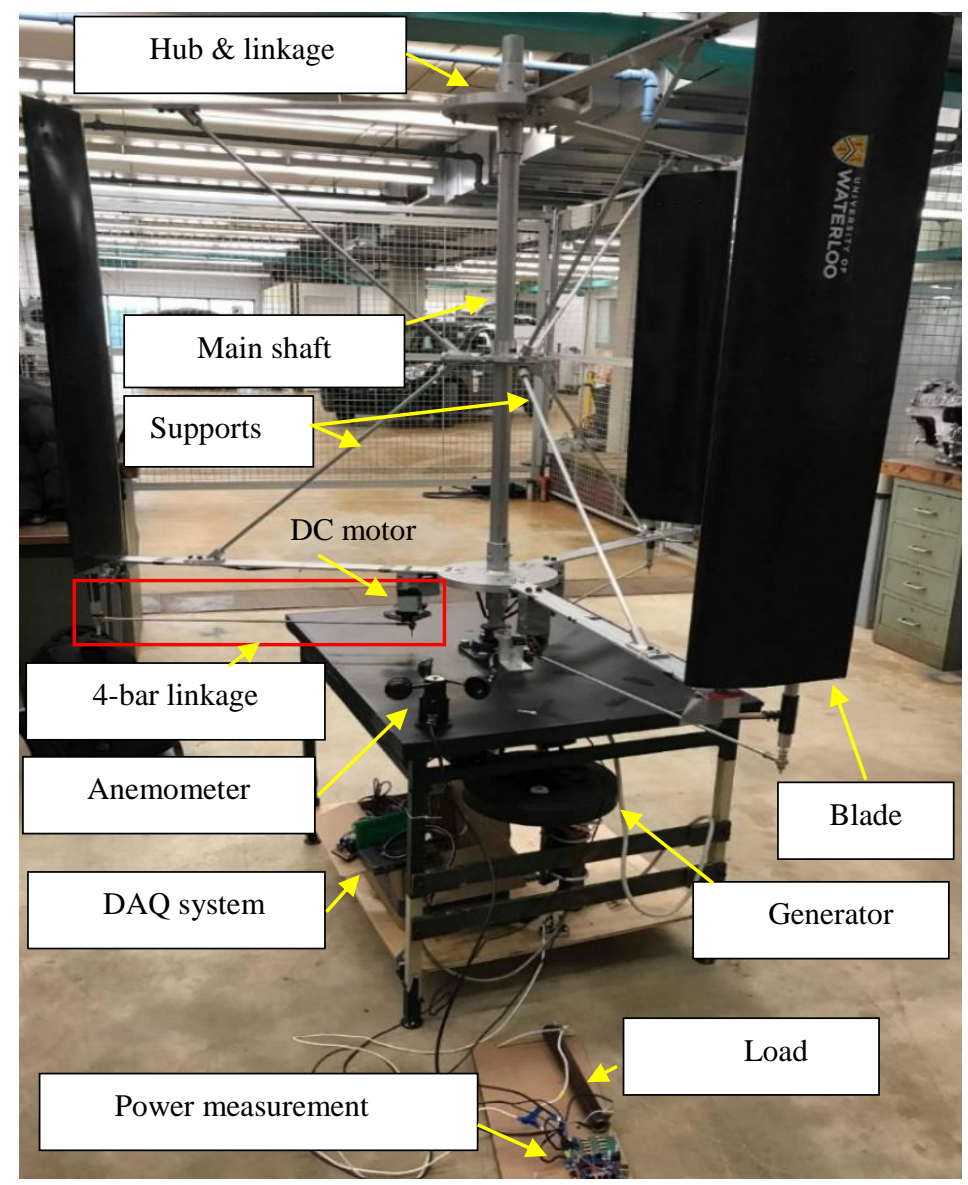

Figure 6.6. Final assemble of the H-type VAWT.

The blade pitching mechanism is the final functional requirement, which will be discussed in next section including more details about the hardware and software for the proposed pitch control system. 


\subsection{Design and implementation of blade pitch control system for an $H$-type VAWT}

Figure 6.7 shows a diagram of the overall experimental pitch control system setup used for validating the effectiveness of the Hybrid controller proposed in Chapter 4. For each blade, the pitch angle is controlled by a closed loop Hybrid controller, which sets a reference angle for each blade according to the instantaneous rotor angle. Then the Hybrid controller tries to set the actual blade pitch angle, measured by blade encoder to track the reference signal with minimal error. Each blade angle is controlled independently using a four-bar linkage actuated by a DC motor.

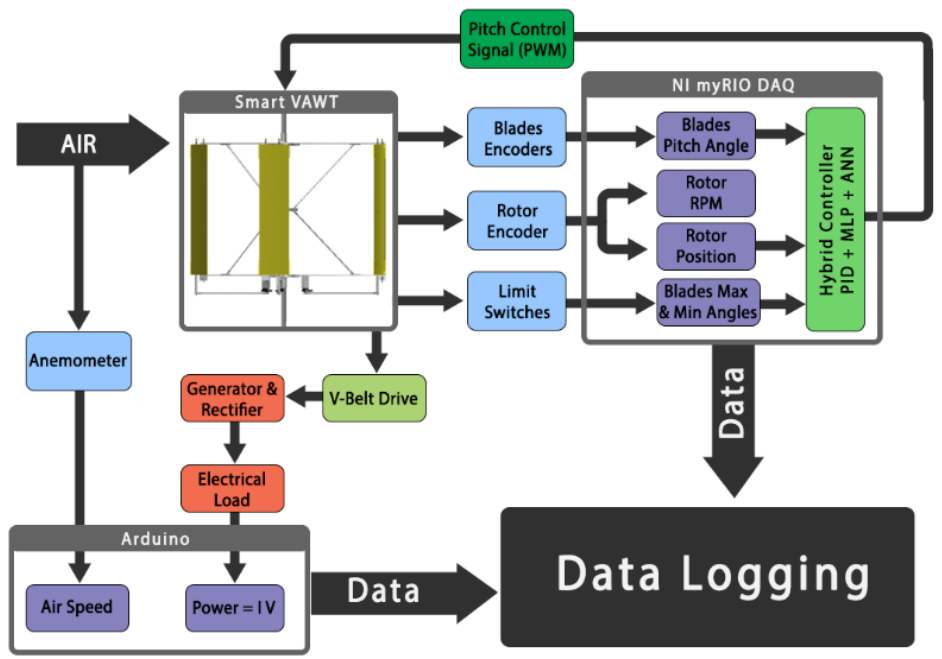

Figure 6.7. Blade pitch control system and data logging.

\subsubsection{Pitch control system hardware}

To facilitate variable pitch command of the blades, a four-bar linkage is proposed which connects the DC motor shaft to the blade pitching shaft as shown in Fig. 6.8. The four-bar linkage CAD design and main dimensions is shown in Fig. 6.9. 


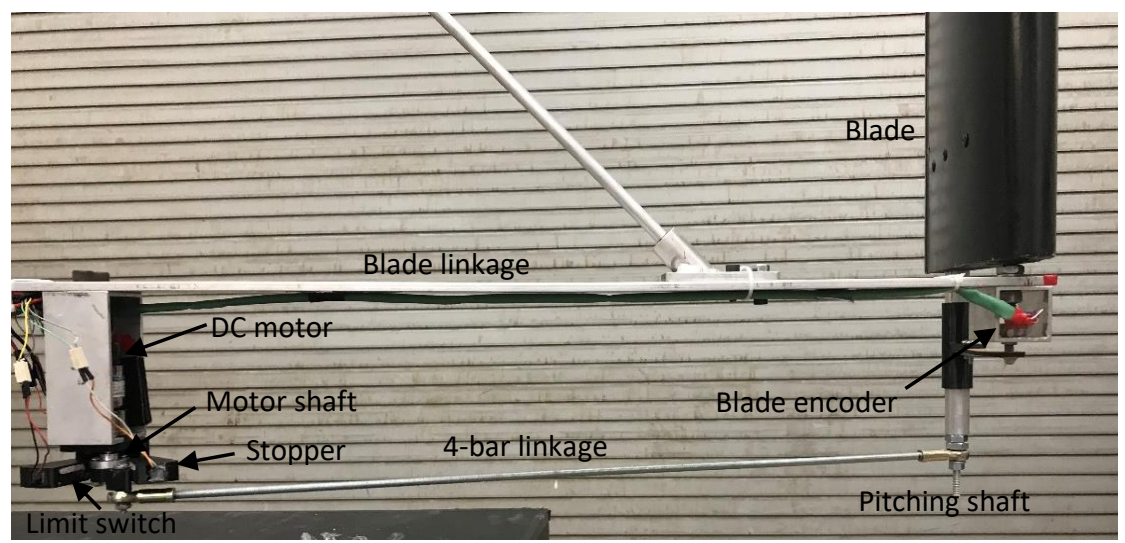

Figure 6.8. Four-bar linkage, DC motor, stopper, and blade encoder experimental setup.

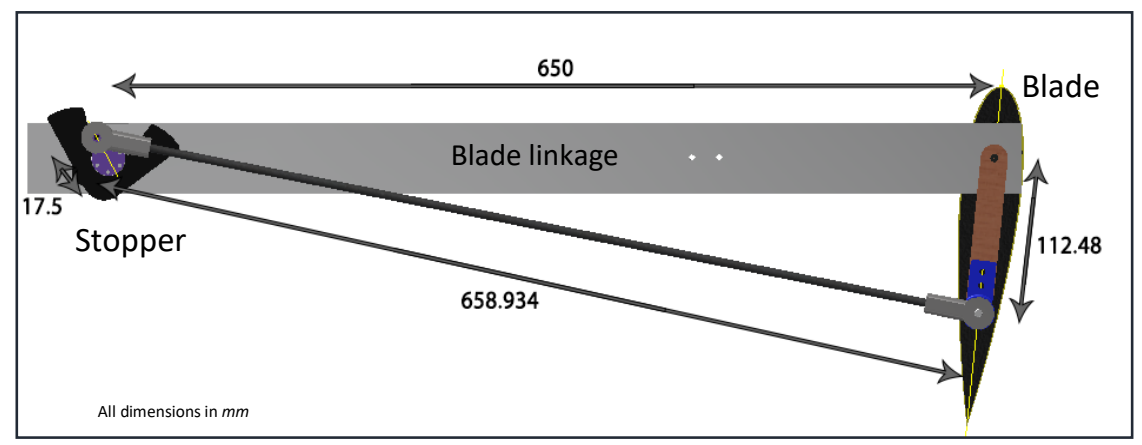

Figure 6.9. CAD design and main dimensions of four-bar linkage system.

The option of using a stepper motor was considered through this research. However, since the blade pitch is allowed to change from $-6^{\circ}$ to $+6^{\circ}$, motor accuracy becomes an important factor. To achieve this accuracy, a DC motor is chosen for more precise positioning and ease of control [93].

Motor sizing refers to the process of selection the correct motor for a given load. In this thesis, the required torque for a DC motor for pitching the blade is calculated based on the blade weight, the blade acceleration, and the angular velocity of blade due to pitching. The required torque can be estimated as follows:

$$
\tau_{\text {req. }}=\text { Force } \cdot l_{\text {blade }}=\frac{m_{\text {blade }} \cdot \ddot{\beta} \cdot l_{\text {blade }}}{2}
$$

where $m_{\text {blade }}$ is the blade mass $(2.5 \mathrm{~kg}), \ddot{\beta}$ is the blade acceleration due pitching, $l_{\text {blade }}$ is the distance between the pitching and pivoting blade shafts as shown in Fig. $6.9(112.48 \mathrm{~mm})$. 
Because of the small blade pitch variations, the effect of both blade angular $(\dot{\beta})$ and acceleration $(\ddot{\beta})$ in Eq. (6.1) is neglected. As a result, the required torque to rotate the blade is approximately $0.2 \mathrm{~N} \cdot \mathrm{m}$ in Eq. (6.1). HENKWELL DC motor (main parameters are listed in Table $\mathrm{C} 1$ in Appendix C) is selected for this experimental setup based on the torque required to rotate the blades for which the motor torque is $7.2 \mathrm{~kg}-\mathrm{cm}$ (approximately $0.706 \mathrm{~N} \cdot \mathrm{m}$ ).

Figures 6.8 and 6.9 also show the DC motor Aluminum housing, stopper, and two limit switches, which are placed at both sides of the stoppers mounted under the lower linkage for each blade.

Figure 6.8 also shows the blade encoder (potentiometer) mounted at the end of blade linkage that is used as a position feedback sensor.

\subsubsection{Data Acquisition system interface (DAQ system)}

The H-type VAWT prototype is equipped with a data acquisition system (DAQ). In this thesis, the data are processed using a Virtual Instrument (VI) developed using LabView software. The NI myRIO-1900, which is connected to LabView wirelessly, is also used as a DAQ device to interface the system hardware with the computer.

\subsubsection{Wiring and PCB fabrication}

A slip ring is used to allow the transmission of power and electrical signals from DC motors, limit switches, and potentiometers to the DAQ system through three holes on the rotating main shaft. Figure 6.10 shows the PCB (printed circuit board) which is interfaced with the DAQ system. The main PCB components are motors, motor drivers, 4-resistances (100 ohm), and $10 \mu \mathrm{f}$ capacitors. Also, more details about the slip ring are in Section C2 in Appendix C. 


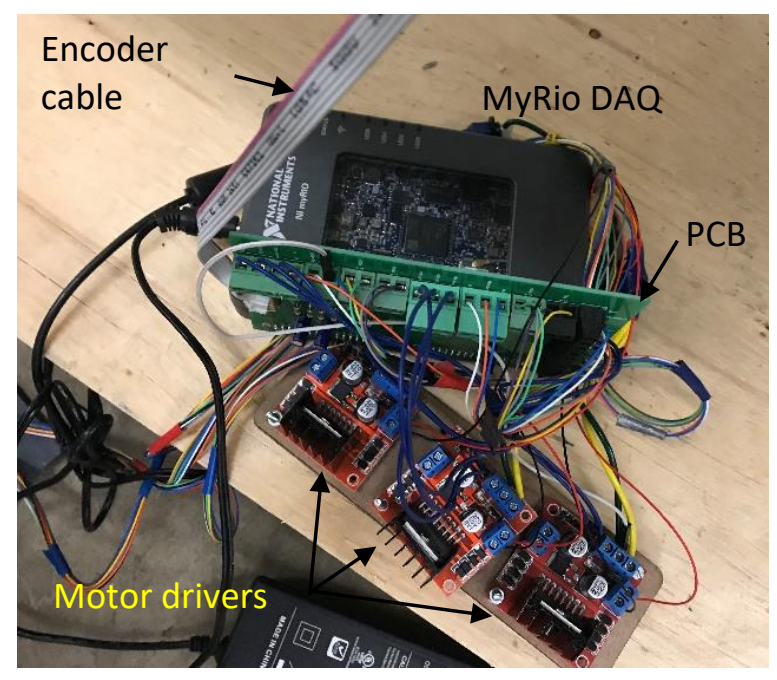

Figure 6.10. PCB for the control system.

\subsubsection{Hybrid controller implementation}

The Hybrid controller proposed in this thesis consists of an MLP-ANN and PID controller combination which uses the pitch angle error $(e)$ as an input signal produced by comparison between reference obtained from CFD results in Chapter 3, and actual pitch angles. However, the reference pitch angle variations are sometimes small, hence, the controller is unable to track them. Therefore, these small changes are adjusted to be greater than or equal to $4^{\circ}$. Also, the sharp edges due to the pitch angle changes are also smoothed for each blade. Figure 6.11 shows comparison between both the original and new adjusted reference pitch angles.
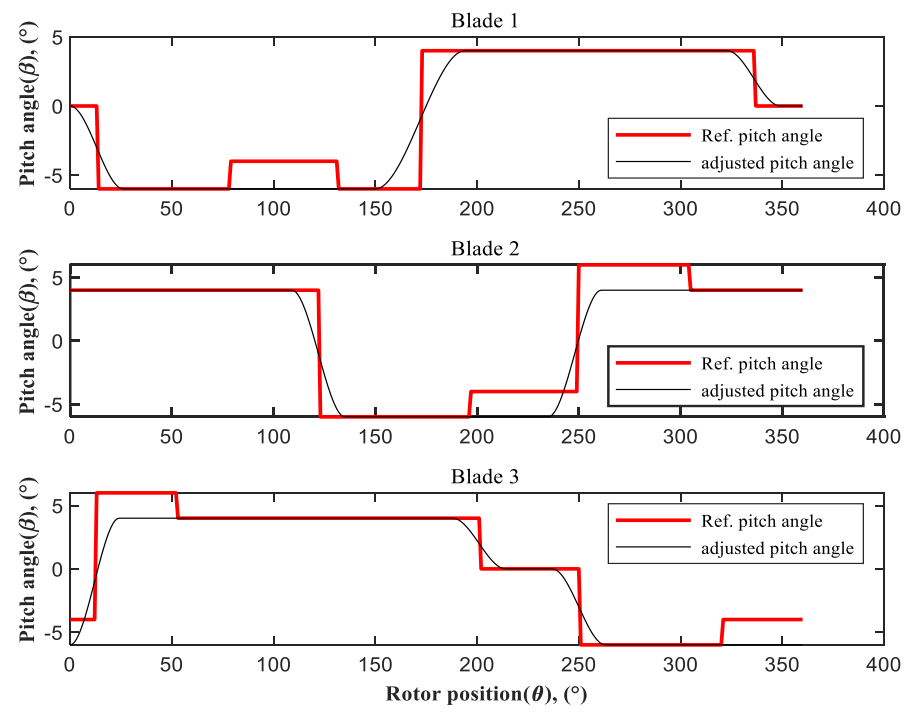

Figure 6.11. Original and adjusted reference pitch angle. 
For the experimental validation of the proposed controller, a Matlab script in VI is developed as shown in Fig. 6.12.

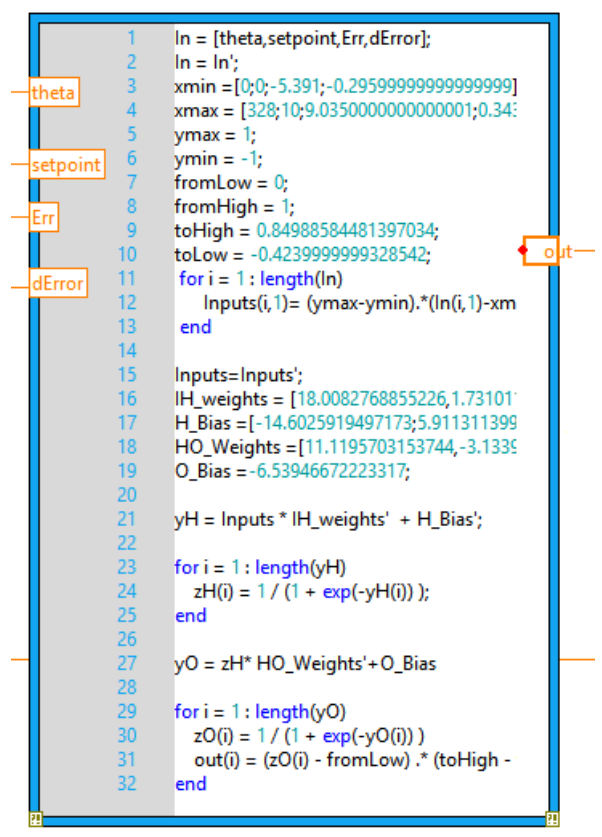

Figure 6.12. Matlab script for the MLP-ANN implemented in LabView.

The adjusted weights, bias, pre-processing and post-processing values are obtained from MLP-ANN Matlab/Simulink toolbox which is discussed in Chapter 4. Figure 6.12 also shows that step-point $\left(\beta_{r e f}\right), \theta$, error $(e)$ and its derivative $(\dot{e})$ are used as input data while the output is the MLP-ANN signal.

Table 6.1 shows the gains for PID controller for each blade, which are tuned manually by using the front panel of LabView software. The output of the MLP-ANN is then combined with the PWM output of the PID to provide the final control command to each blade.

Table 6.1. PID gains for each blade.

\begin{tabular}{cccc}
\hline & $\boldsymbol{K}_{\boldsymbol{p}}$ & $\boldsymbol{K}_{\boldsymbol{i}}$ & $\boldsymbol{K}_{\boldsymbol{d}}$ \\
\hline Blade 1 & 0.12 & 100 & 0.043 \\
Blade 2 & 0.088 & 100 & 0.009 \\
Blade 3 & 0.1 & 100 & 0.01 \\
\hline
\end{tabular}




\subsubsection{Feedback loop of the blade pitching system}

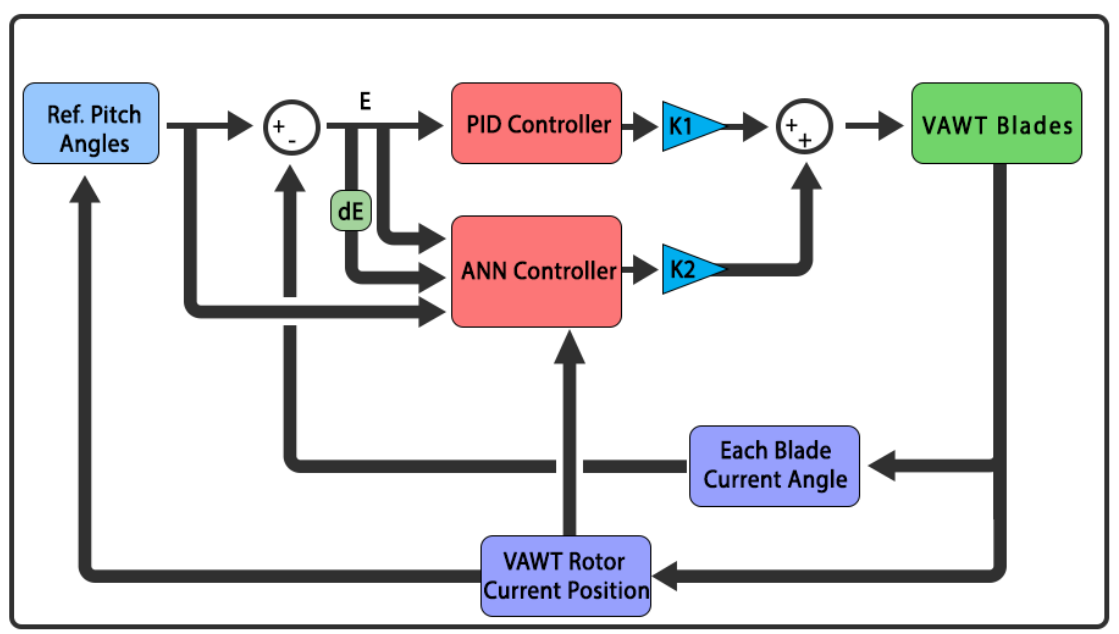

Figure 6.13. A block diagram of the closed-loop pitch control system for one blade.

Figure 6.13 shows the block diagram for the feedback Hybrid controller for one blade. This basic feedback loop of sensing, controlling and actuation is represented by two main processes: initialization and main loop (see Section C3 in Appendix C).

\subsubsection{Experimental measurements}

In order to investigate the effect of blade pitching on performance of H-type VAWT in terms of the power output as well as self-starting capability, some parameters such as the wind speed, the rotor speed (RPM), the actual pitch angles, and the PMSG current and voltage should be measured by using specific sensors which are interfaced with the DAQ system.

\section{- Rotor speed (RPM) measurement}

The pitch angle is a function of rotor position, $\beta=f(\theta)$, hence, it is important to determine the rotor position and rotor RPM. The absolute rotary encoder is used herein for measuring the rotor speed (in rpm). It produces 1024 pulses per revolution (PPR). Figure 6.14 shows the rotor main shaft coupled with the encoder shaft by using two 3D printed gears. 


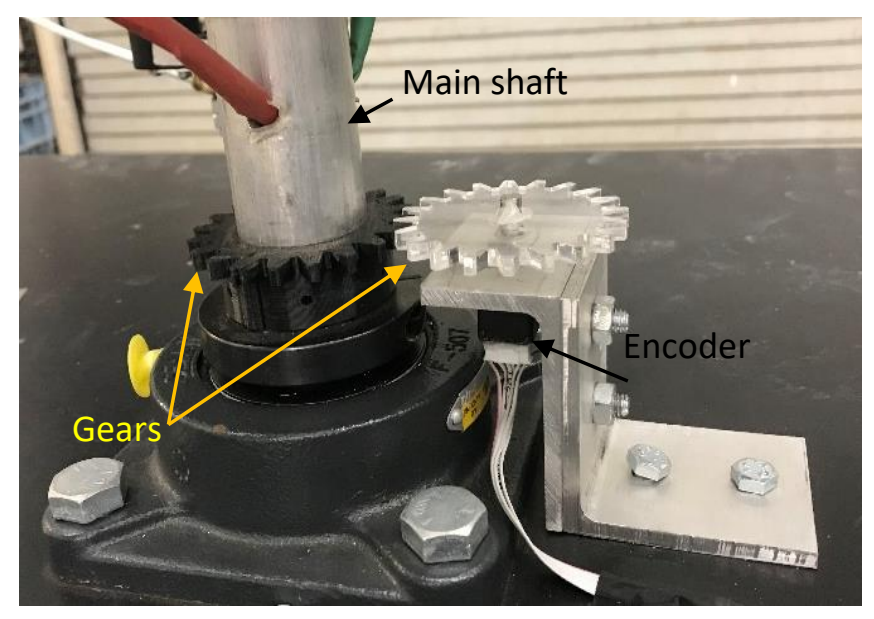

Figure 6.14. Absolute rotary encoder experimental setup.

\section{- Wind speed measurement}

The power characteristics of wind turbines are evaluated based on some important parameters such as, wind speed. In this thesis, an anemometer sensor, which has a resolution of $0.1 \mathrm{~m} / \mathrm{s}$, is used to measure wind speed. This sensor is also capable of measuring wind speeds up to about $70 \mathrm{~m} / \mathrm{s}(252 \mathrm{~km} / \mathrm{h})$

\section{- Power output measurement}

In this thesis, a low RPM 3-phase permanent magnet synchronous wind turbine generator (PMSG) is used for the H-type VAWT to produce a 3-phase AC voltage of $0.608 \mathrm{~V}$ per RPM. The 3-phase PMSG produces Alternating Current (AC) that is converted to Direct Current (DC) by a 3-phase bridge rectifier. The resulting rectified DC voltage is connected to 250-ohm power resistor, which is used as a load for drawing electricity from the PMSG. The DC voltage based on the H-type VAWT RPM can be calculated as follows:

From PMSG specifications (see $\mathrm{C} 1$ in Appendix C), the root mean square voltage $\left(V_{r m s}\right)$ :

$$
V_{\text {rms }}=0.357 * R P M_{\text {gen }} .
$$

where $R P M_{\text {gen. }}$ is the generator speed. The DC voltage $\left(V_{D C}\right)$ after full wave rectification is calculated as [94] 


$$
V_{D C}=\frac{3 \sqrt{2}}{\pi} * V_{r m s}
$$

The V-Belt drive reduction ratio:

$$
\text { Reduction Ratio }=\frac{D_{\text {pulley } 1}}{D_{\text {pulley } 2}}=\frac{11.75}{2}=5.875
$$

Therefore:

$$
\begin{gathered}
V_{D C}=\frac{3 \sqrt{2}}{\pi} * 0.357 * \underbrace{\text { Reduction Ratio } * \text { RPM }}_{R P M_{\text {gen }}} \text { rotor } \\
V_{D C} \approx 2.83 * R P M_{\text {rotor }}
\end{gathered}
$$

The average rotor speed $\left(R P M_{\text {rotor }}\right)$ for the H-type VAWT based on the experimental results is about $20 \mathrm{rpm}$, so the voltage will be around $57 \mathrm{DC}$.

Figure 6.15 shows that power circuit which includes the current and voltage sensors along with an Arduino as a data logger. The voltage limits on $\mathrm{I} / \mathrm{O}$ pins are only between 0 and $5 \mathrm{~V}$. Therefore, the voltage divider circuit is used to map the high input DC voltage to be in Arduino voltage limits. Also, the wind speed sensor is connected to Arduino to collect wind speed data.

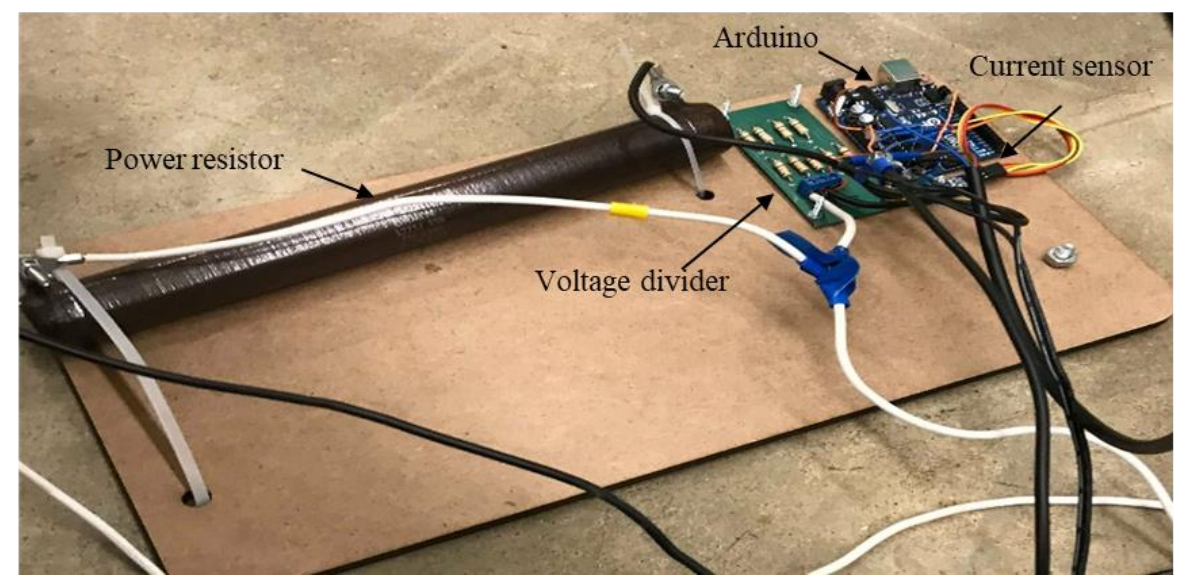

Figure 6.15. Power output measurement circuit. 


\subsection{H-type VAWT experimental results}

Because of lack of availability of an open-wind tunnel, the H-type VAWT including the pitch control system is extensively tested outdoor by using a pickup truck as an alternative. The test setup is shown in Fig. 6.16.

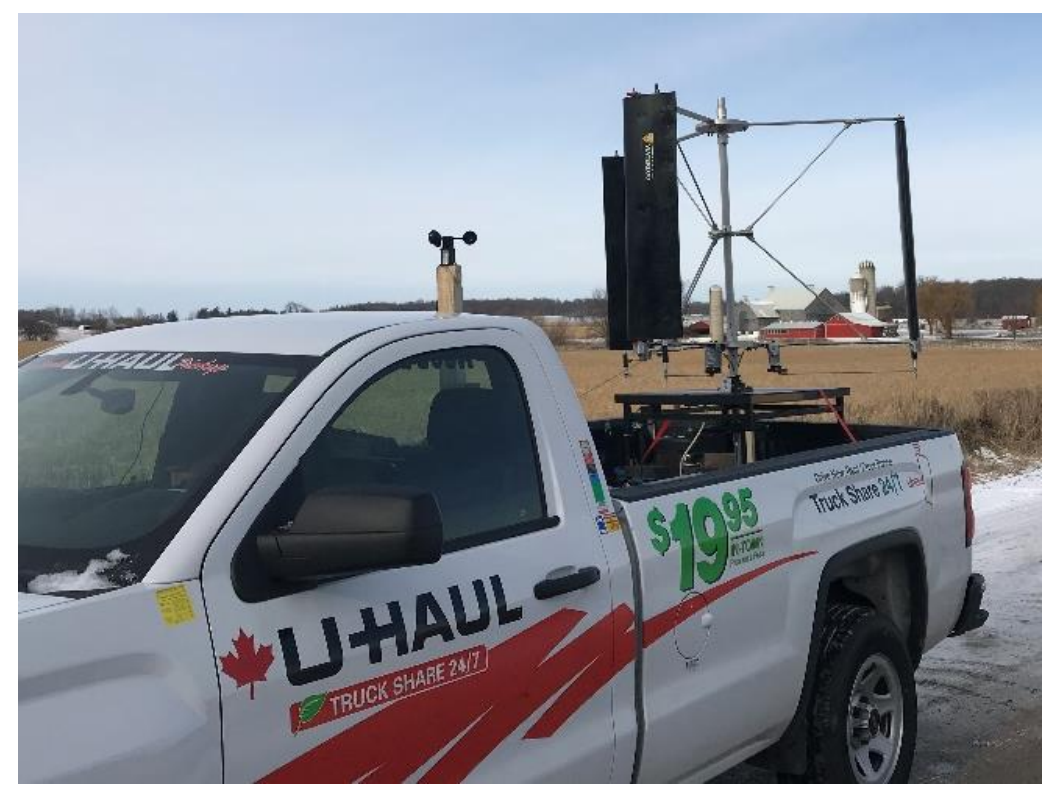

Figure 6.16. A pickup truck for H-type VAWT experimental setup.

A pickup truck is driven down a remote public roadway without any overhead obstructions, such as overpasses, transmission lines, or traffic lights. Tests are conducted under different operating conditions.

All apparatus are mounted on the back of a pickup truck and connected to the DAQ system for data monitoring. The H-type VAWT is placed near the rear to reduce the effect of airflow disturbance by the truck. The anemometer is also mounted on the pickup roof on approximately $2 m$ from upwind of the rotor. An external AC power supply is used to power the electrical equipment, such as DC motors and data loggers. For data collection, all data are recorded continuously each 6 ms using myRIO DAQ system.

The outdoor trials are divided into three main experimental tests based on the purpose of test: the preliminary test is conducted to examine the mechanical structure of the H-type VAWT prototype and its rotor speed. In the first test, power output, self-starting capability, 
and the proposed control effectiveness of the H-Type VAWT prototype are investigated in both fixed and variable blade pitch configurations. To confirm the findings, the second test is performed by repeating the first test under different wind conditions. The operational reliability of the H-type VAWT is monitored in the preliminary test, which shows that the Htype VAWT mechanical structure is stable at different rotor speeds, ranging from 18 to 48 rpm.

In this thesis, the effect of three main speed components is considered for experimental analysis:

- Ground wind speed: it is the local speed of the wind relative to the ground that is collected including its direction from the University of Waterloo weather station [95].

- Air speed: it is the speed of the wind relative to the vehicle which is measured by using the anemometer when the vehicle is traveling in different directions. The anemometer can also measure the ground wind speed when the vehicle is parked.

- Vehicle speed: it is the speed of the vehicle, ranging from 60 to $80 \mathrm{~km} / \mathrm{hr}$.

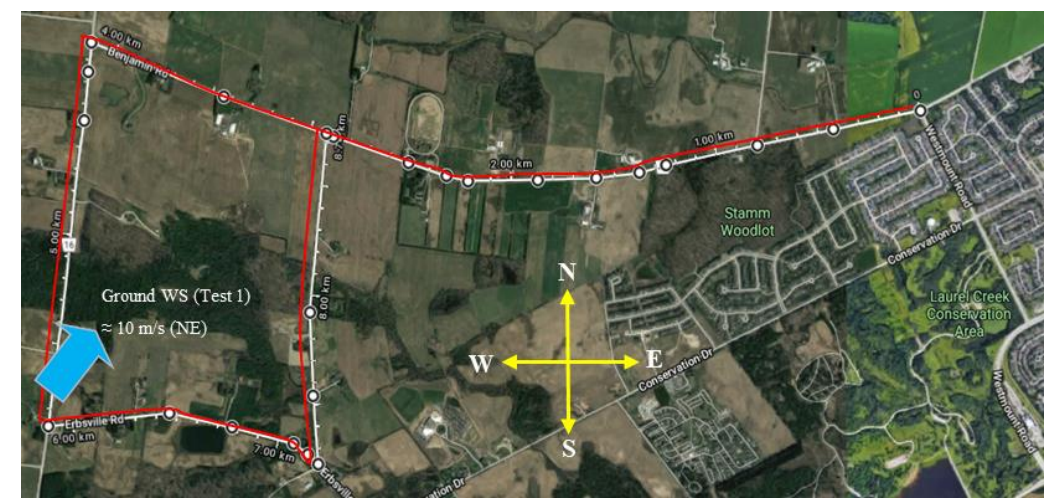

Figure 6.17. Main road for Test 1 and Test 2 and ground wind speed for Test 1.

For the first test, the average ground wind speed was around $9.6 \mathrm{~m} / \mathrm{s}$ while it is only around $1 \mathrm{~m} / \mathrm{s}$ for the second test. The main road for first and second tests is shown in Fig. 6.17 . 


\subsubsection{Representing wind variations}

The variations in wind speed are usually described by using statistical tools such as Weibull and Normal distributions. This experimental study uses the normal probability plot (P-plot) [96] to assess whether or not a data set is approximately normally distributed. Based on the assumption that the data come from a normal distribution, the deviations of wind speed data from the mean are arranged in ascending order, then, standardized by using $\mathrm{z}$-scores in Eq. (6.7).

$$
z=\frac{x_{i}-\mu}{\sigma_{s d}}
$$

where $x_{i}$ is the observed data, $\mu$ is the mean of data, and $\sigma_{s d}$ is the standard deviation of data. Figure 6.18 shows the P-plot for the wind speed data for both outdoor tests. It can be seen that the data set in both cases deviate from the straight lines, specifically, from the bottom left and top right of lines. It can be concluded that the data does not come from normal distribution due to the outliers and a general lack of fit. Also, because the anemometer recorded the wind speed when the pickup truck is travelling or parked, the collected data has a mix of ground and air wind speeds.

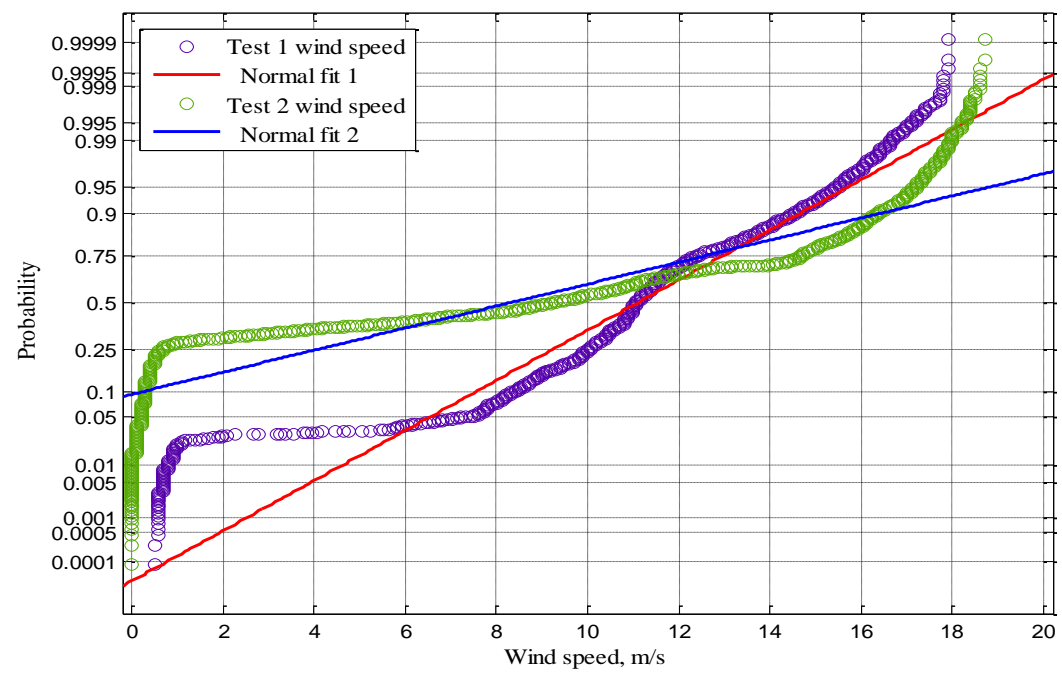

Figure 6.18. Normal probability plot (P-plot) for wind speed data.

Therefore, data pre-processing is applied to remove the extreme outliers. After data preprocessing, the data set approximately follows a normal distribution as shown in Fig. 6.19. 


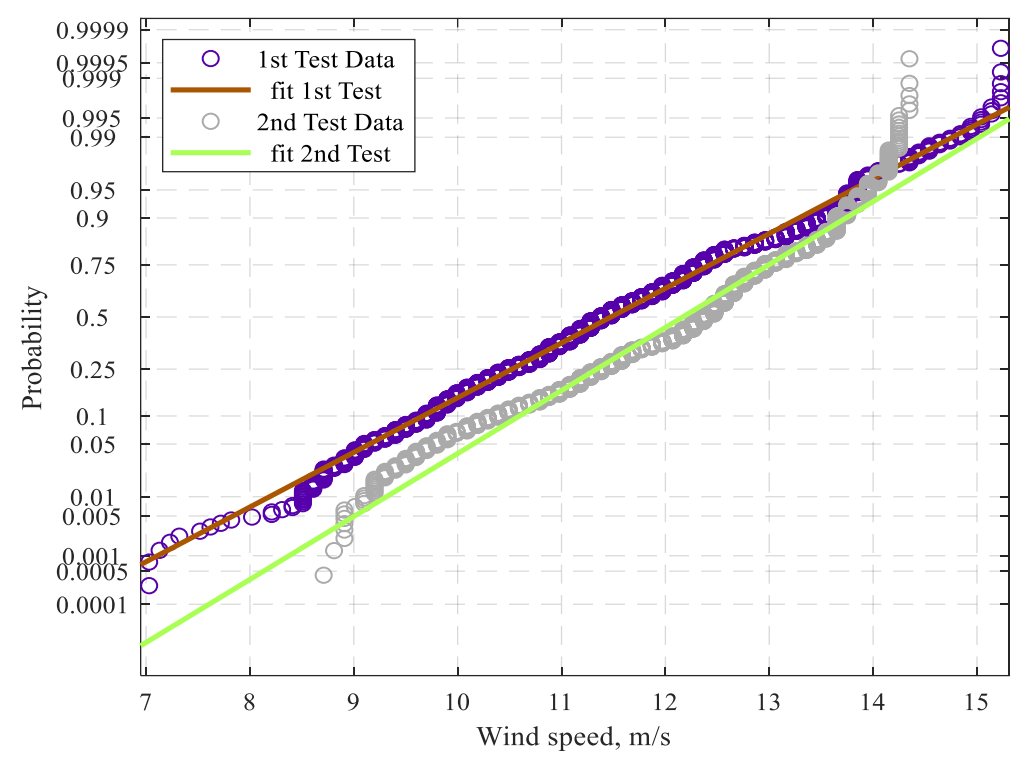

Figure 6.19. P-plot for processed wind data.

\subsubsection{Power curve of the H-type VAWT}

The measured power output for wind turbines at different wind speeds is characterized by a power curve to investigate the efficiency of wind turbines. For both fixed and variable pitch angle configurations, the power generation can be normalized by using the per unit system $(p u)$, which is calculated by dividing the actual power output by the nominal power output of the wind turbine [97]. Figure 6.20 shows the power generation in $p u$ versus the wind speed in $m / s$ for the H-type VAWT

In general, there are no significant differences in terms of the power output for all cases in both tests as shown in Fig. 6.20. However, the effect of wind direction and high magnitude of the average ground wind speed, which was close to the average air speed of $10.159 \mathrm{~m} / \mathrm{s}$, is noticeable on the power output in the first experimental test for both fixed and variable pitch angle cases. To be more specific, the H-type VAWT produces lower power output as shown in Fig. 6.20, which is close to 0, at wind speed ranging from 10 to $12 \mathrm{~m} / \mathrm{s}$ compared with the second test. This may be due to the effect of air resistance on both sides of blades. For variable pitch angle in both experimental tests, a small amount of power output at wind speed 
ranging from 4 to $7 \mathrm{~m} / \mathrm{s}$ is produced by the H-type VAWT as shown in Fig. 6.20, which means that the self-starting capability is enhanced by using the proposed blade pitching technique. The measured power output is fitted using nonlinear curves (black curves) for all tests as shown in Fig. 6.20.

The power output fluctuations are the main challenge of wind energy industry in terms of design and utilization [98]. The results of both tests are analyzed based on a short time scale. A short-term wind fluctuation analysis plays a key role in describing the aerodynamic behavior and efficiency of wind turbines [98][99]. The effect of short-term wind fluctuation on power output can be clearly seen in Fig. 6.21 where there is a rapid drop in power output within a few seconds due to wind speed variations.

Figure 6.21 also shows the filtered power generation curves for both tests in fixed and variable pitch angle configurations. For data filtering, a moving-average filter is used herein to reduce the power fluctuations by determining averages along all data for all tests. Moreover, instantaneous power output, which is less than or equal to zero, is rejected. The areas below all of the power curves displayed in Fig. 6.21 represent the total gross energy harnessed by the H-type VAWT for fixed and variable pitch cases. Numerical integration using the trapezoidal rule is performed to calculate the total gross energy. The results showed that the total energy is increased by $21 \%$ and $22.9 \%$ by using the proposed blade pitching technique for the first and second tests, respectively. The power consumed by the control system equipment, which was around 9 watts, is subtracted when calculating this improvement percentage. 

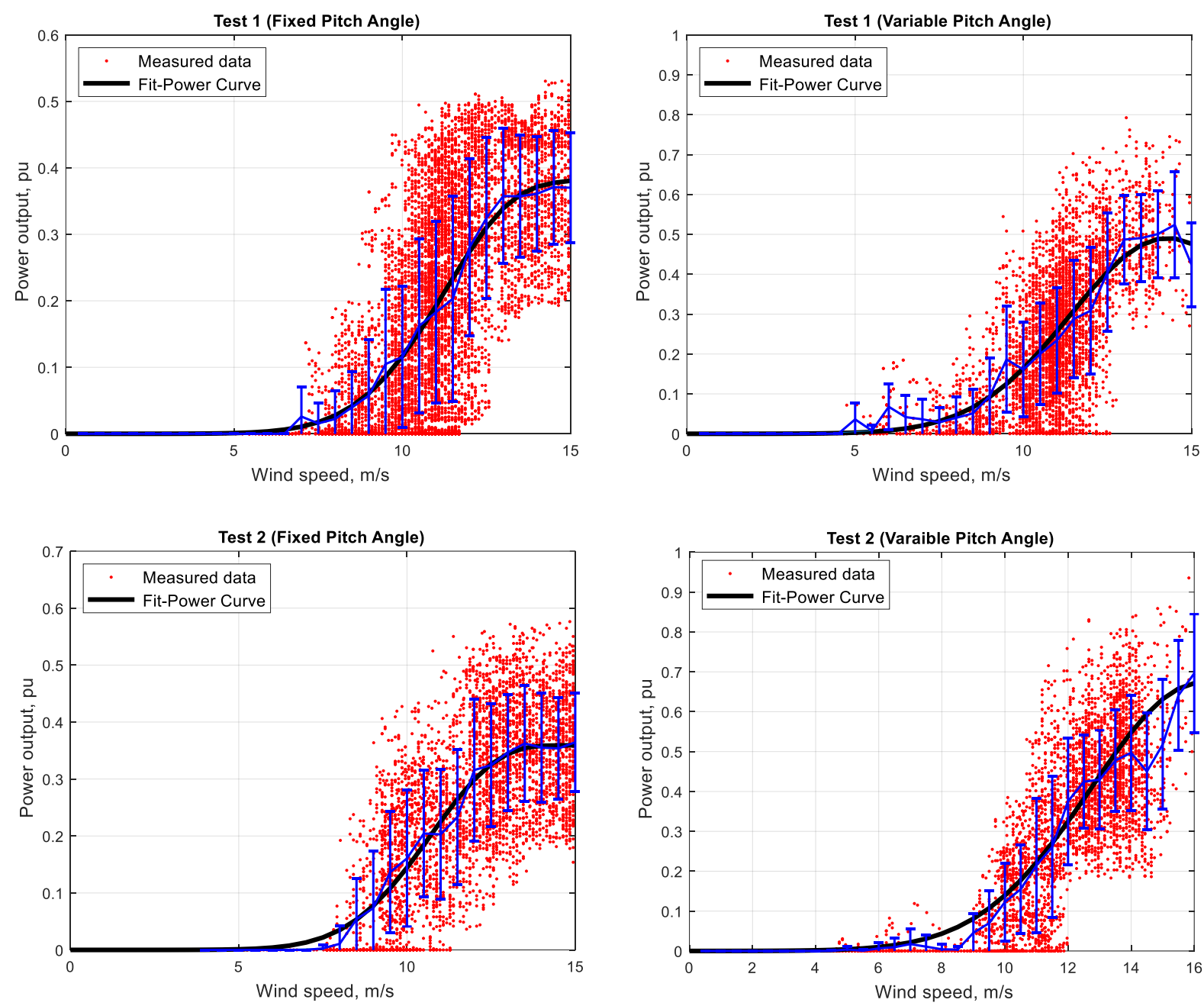

Figure 6.20. The H-type VAWT power generation of first and second tests in both fixed and variable pitch configurations.
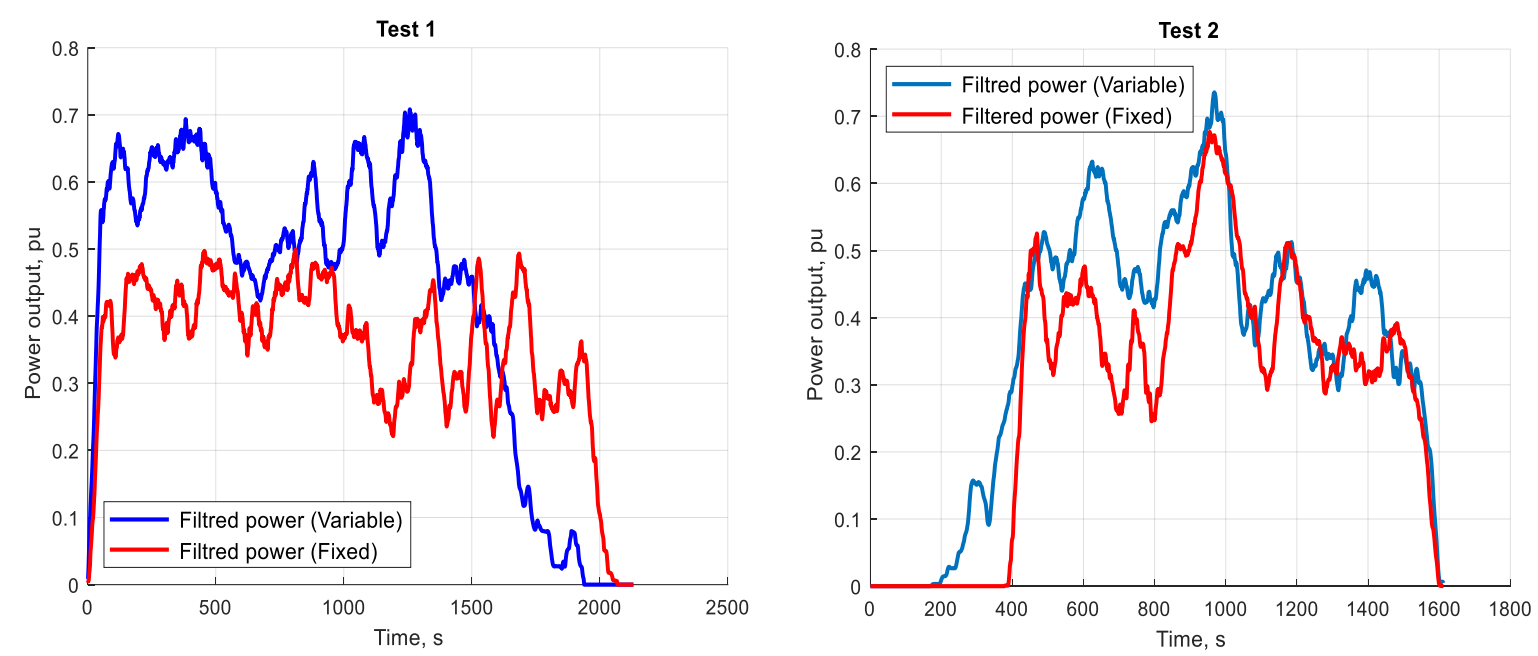

Figure 6.21. Filtered power generation curves for both tests in fixed and variable pitch angle configurations. 
The wind turbine power generation is associated with rotor speed (RPM) variations. The rotor speed is measured by using an absolute encoder for all cases as shown in Fig. 6.22. In the fixed pitch angle case, high variations are generally observed for both tests; namely, RPMs for the first test, as shown in Fig. 6.22 (a). This may be due to the effect of the high ground wind speed magnitude and its direction. These variations are, however, reduced by blade pitching technique as shown in Fig. 6.22 (b). Consequently, the power output fluctuations are also reduced in the variable pitch angle case as shown in Fig. 6.21. In the variable pitch angle case, Fig. 6.22 (b) also shows that the RPM peaks are at about 48 rpm and $40 \mathrm{rpm}$ for the first and second tests, respectively, while they are less than $35 \mathrm{rpm}$ for both tests in the fixed pitch angle case. This means that the rotor speed is increased by the blade pitching, which is attributed to higher rotor torques due to blade pitching.
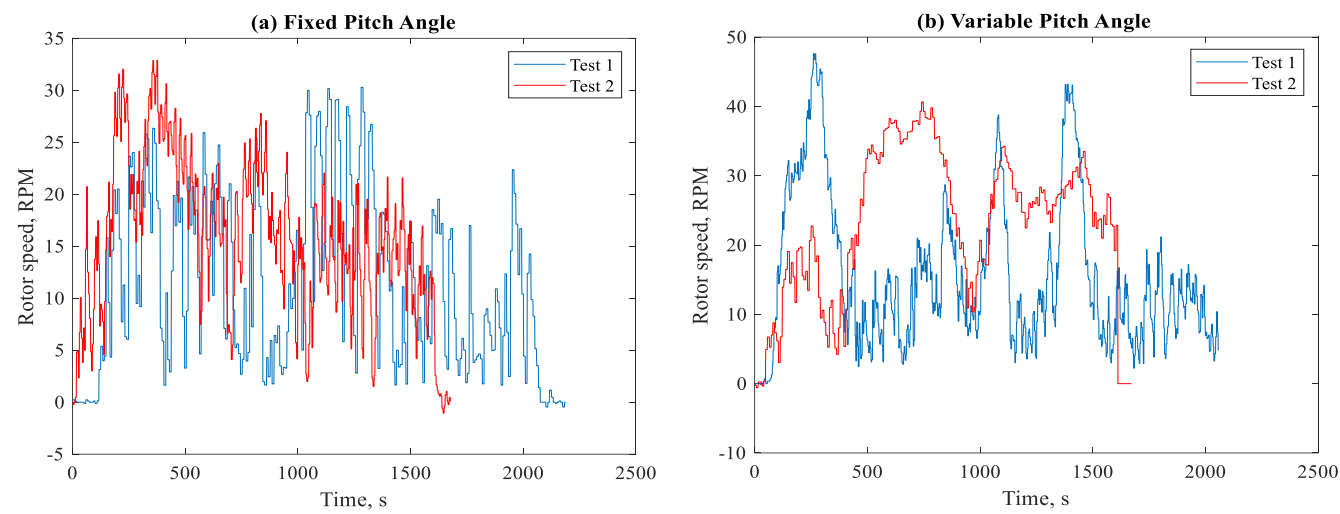

Figure 6.22. The H-type VAWT rotor speeds for both tests.

\subsubsection{CFD Numerical validation}

The fixed pitch angle case in first test is chosen for the validation because it has a good power fit curve based on the results in Fig. 6.20. The experimental results are validated numerically by repeating the CFD simulation for a 2D H-type VAWT model at different TSRs which are calculated from experimental results. The experimental power coefficient $\left(C_{p}\right)$ values can be determined for fixed pitch angle case by using the fitted power curves shown in Fig. 6.20. 


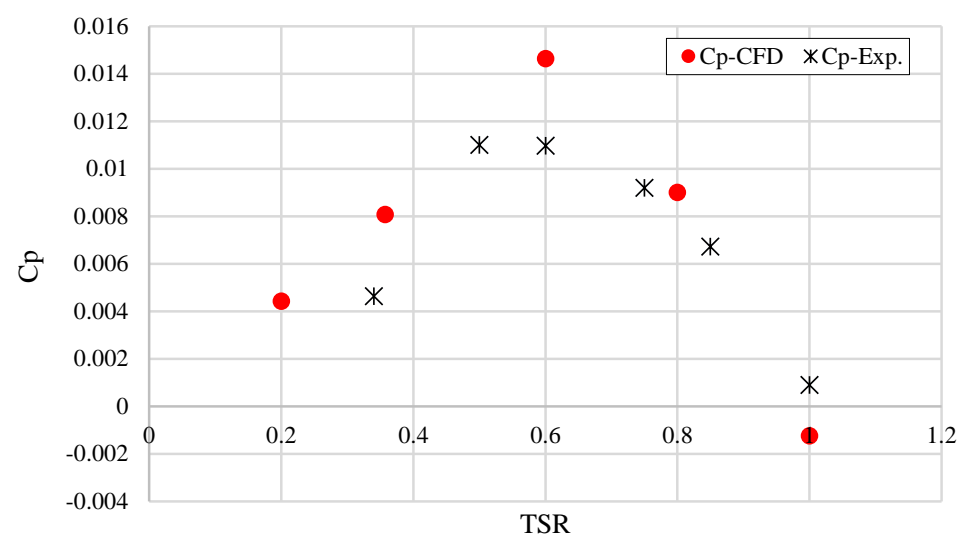

Figure 6.23. Power coefficient comparison between the experimental and CFD results.

The comparison between the experimental and numerical results is shown in Fig. 6.23. Although the power coefficient values are overestimated by the CFD model for a 2D H-type VAWT, a reasonably good agreement can still be seen between the CFD and experimental results. The Fréchet distance [100] is used to measure the similarity between $C_{p}$ curves in both experimental and CFD cases as shown in Fig. 6.24.

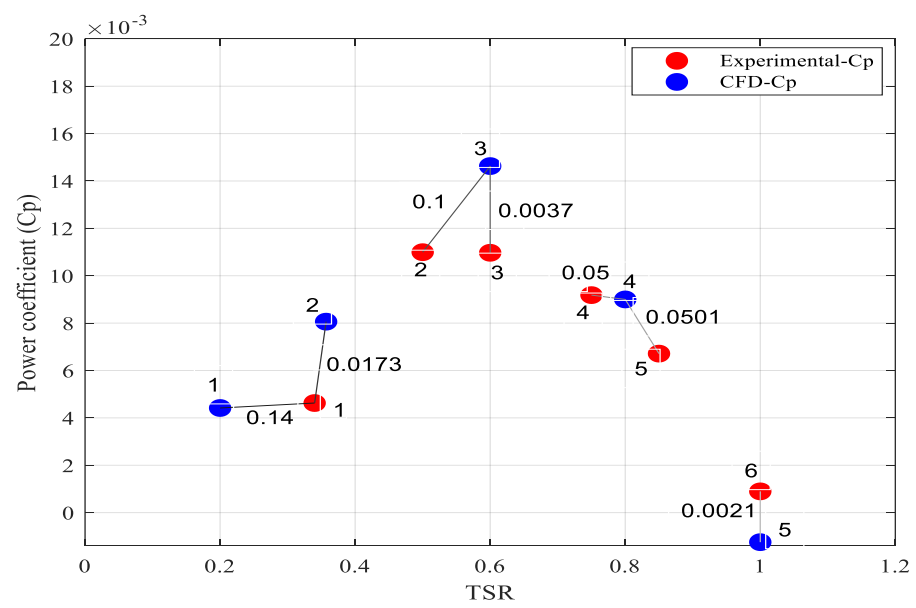

Figure 6.24. The Fréchet distance of experimental and numerical (CFD) power coefficient curves.

It can be seen that the distance between all points for both curves is small which means the both curves are slightly similar.

\subsubsection{Pitch angle control system effectiveness}

The effectiveness of blade pitch control system for each blade is examined by several indoor tests as well as outdoor tests. The H-type VAWT prototype is easy to rotate by using two mid-size fans if the v-belt in pulley system is detached to the generator. As a result, the 
Hybrid pitch control system is extensively tested indoor by using different user-specified percentages of PID and MLP-ANN output signals.

In order to avoid the instability region as shown in Fig. 5.7, the proposed control system is tested at low TSRs less than 1. For each blade, the response of Hybrid controller for three revolutions at different RPMs is compared to the reference pitch angles as shown in Fig. 6.25. Although the ability of obtaining a good set point tracking performance for the Hybrid controller is challenging because of the small change in pitch angles, it can be seen that using large percentage of PID output signal $(80 \%)$ in the first outdoor test exhibited a reasonable pitch angle response for only low RPMs. Overshoots are observed at high RPMs. However, nonlinear dynamics of the H-type VAWT, which is evident at high RPMs, is overcome by using large percentage of $80 \%$ MLP-ANN output signal in the second outdoor test in order to achieve desirable blade pitch control performance. For the first blade, the overshoot is generally lower than in the second and third blade control response because the PID controller for the first blade uses a proportional gain greater than those for the other two blades as shown in Table 6.1 . 
Blade 1
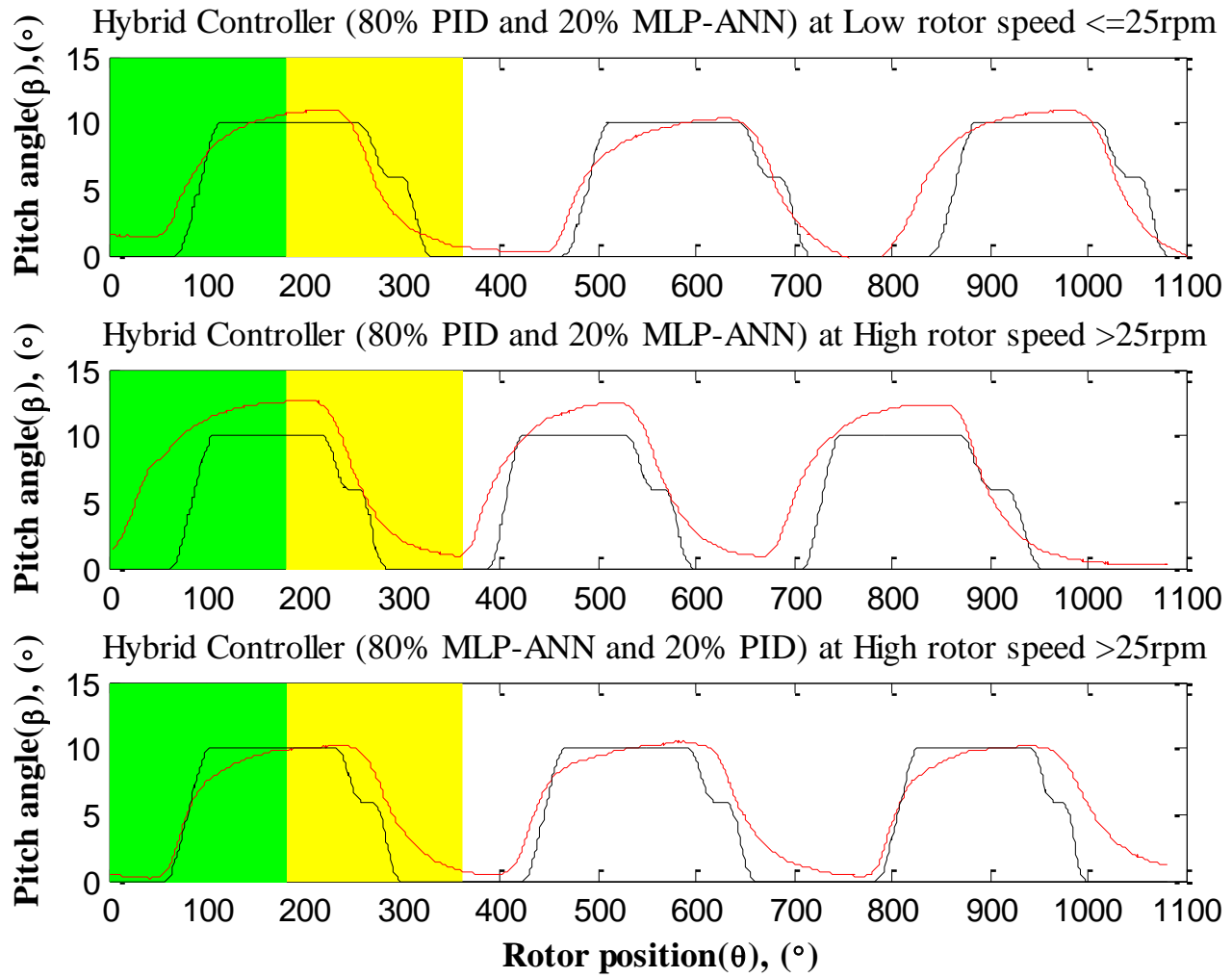

Blade 2

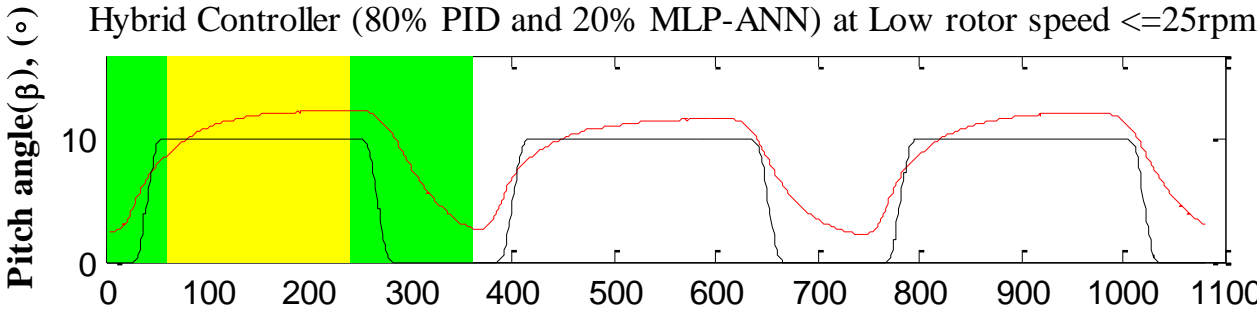

( Hybrid Controller (80\% PID and 20\% MLP-ANN) at High rotor speed $>25 \mathrm{rpm}$

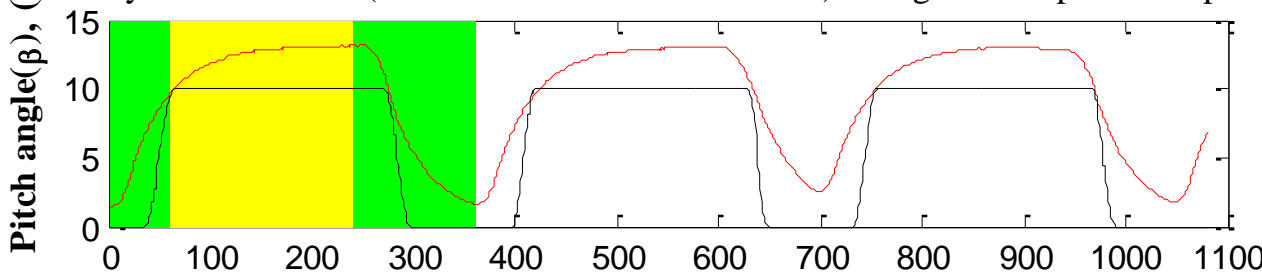

( Hybrid Controller (80\% MLP-ANN and 20\% PID) at High rotor speed $>25 \mathrm{rpm}$

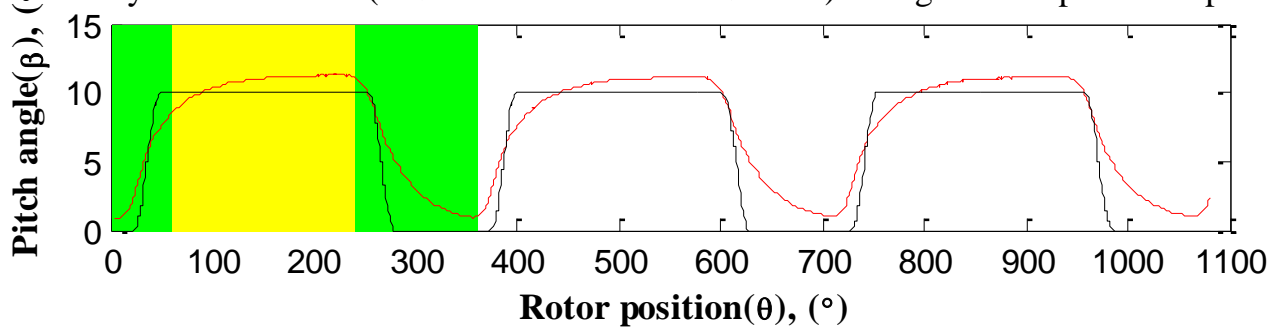


Blade 3
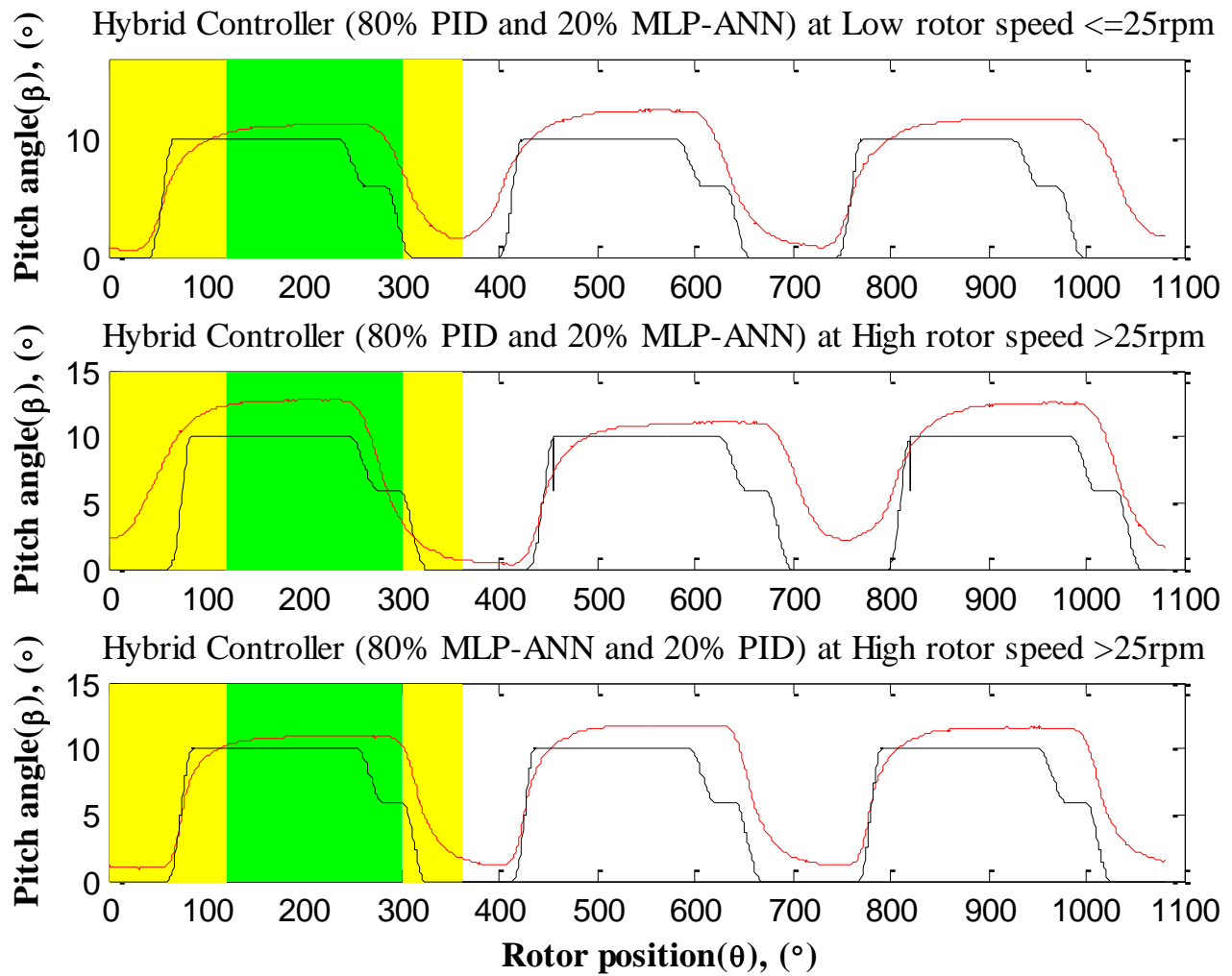

Figure 6.25. Response of the Hybrid controller for each blade at different RPMs for three revolutions.

The differences between reference pitch signal and measured pitch signal are determined by calculating the root mean square error (RMSE). For all cases, RMSEs in Table 6.2 show that the Hybrid controller which uses $80 \%$ MLP-ANN is able to reduce the overshoot at high RPMs by comparison to the Hybrid controller with $80 \%$ of the PID signals. However, $80 \%$ of the PID can only handle overshoot at low RPMs. Also, a good pitch response by the Hybrid controller can be demonstrated for the second blade attributed to the simple shape of the reference signal compared to the first and third blades.

Table 6.2. RMSE of the Hybrid controller in different weights.

\begin{tabular}{cccc}
\hline RMSE & $\begin{array}{c}\text { 80\% PID \& 20\% } \\
\text { MLP-ANN at low RPMs }\end{array}$ & $\begin{array}{c}\text { 80\% PID \& 20\% } \\
\text { MLP-ANN at high RPMs }\end{array}$ & $\begin{array}{c}\text { 80\% MLP-ANN \& 20\% } \\
\text { PID at high RPMs }\end{array}$ \\
\hline Blade 1 & 3.2181 & 3.2741 & 1.7961 \\
Blade 2 & 1.6629 & 2.8732 & 1.8962 \\
Blade 3 & 2.6248 & 3.0238 & 2.4718 \\
\hline
\end{tabular}


For one rotor revolution, the upstream and downstream regions of the rotor are shown in Fig. 6.25 in green and yellow areas, respectively. These regions are used to investigate the effect of ground wind speed on the effectiveness of pitch control system by determining the peak of pitch control response for all blades. In general, it can be seen that the overshoot occurs for the first and second blades in the downstream region. For the third blade, the overshoot occurs in the upstream region only in the first revolution, but it occurs in the second and third revolutions in the downstream region.

In fact, the downstream region is subject to the influence of ground wind speed and vortex shedding which is produced from the upstream blade. This may be the cause of overshoot of the Hybrid controller response in the downstream region. Overshoot values of Hybrid controller response in the downstream region for all blades are listed in Table 6.3. It can be observed that the overshoots for the Hybrid controller which uses 80\% MLP-ANN at high RPMs are less than those for the other two controllers which use $80 \%$ PID signals at low and high RPMs. Although the derivative parameter $\left(\mathrm{K}_{\mathrm{d}}\right)$ of the PID controller is added, which is listed in Table 6.1, to decrease the overshoot of the Hybrid controller, the controller which uses $80 \%$ PID shows a limited ability to reduce overshoot at high RPMs.

Table 6.3. Overshoot of the Hybrid controller response for the first revolution.

\begin{tabular}{cccc}
\hline $\begin{array}{c}\text { Overshoot } \\
(\text { Ref. pitch=10 }\end{array}$ & $\begin{array}{c}\text { 80\% PID \& 20 \% } \\
\text { MLP-ANN at low } \\
\text { RPMs }\end{array}$ & $\begin{array}{c}\text { 80\% PID \& 20\% } \\
\text { MLP-ANN at high } \\
\text { RPMs }\end{array}$ & $\begin{array}{c}\text { 80\% MLP-ANN \& } \\
\text { 20\% PID at high RPMs }\end{array}$ \\
\hline Blade 1 & 11.04 & 12.7 & 10.17 \\
Blade 2 & 12.4 & 13.15 & 11.17 \\
Blade 3 & 11.33 & 12.8 & 10.9 \\
\hline
\end{tabular}




\subsection{Conclusion}

The effect of blade pitching on an H-type VAWT in terms of both power output and selfstarting capability is numerically investigated, as discussed in Chapter 3 and Chapter 4 . The results showed that the power output and self-starting capability are increased by $25 \%$ and $12 \%$, respectively. In order to validate these results, a full-scale of H-type VAWT with blade pitching capability was built to examine the performance of the proposed Hybrid pitch control system.

The preliminary test was conducted to examine the stability of mechanical structure for the H-type VAWT prototype and its rotor speed. The first test is performed to investigate the effectiveness of proposed Hybrid control while the second test is conducted to confirm the findings of the first test. The results showed that the blade pitching technique improved the power output of the H-type VAWT by about $22 \%$. The effectiveness of the Hybrid control system is also investigated by using different weights of both PID and MLP-ANN controllers at different rotor speeds (RPMs). It concluded that the use of high percentage of MLP-ANN improved the blade pitch control response at high RPMs. However, the PID controller with high percentage reduced the overshoot of pitch control response at low RPMs.

The power coefficient $(\mathrm{Cp})$ curve for the first test is obtained from the measured power output and validated numerically using a CFD model for a 2D H-type VAWT. Although the $\mathrm{CFD}$ results showed overestimation of $\mathrm{Cp}$, there is still a reasonably good agreement between the experimental and numerical $\mathrm{Cp}$ curves. 


\section{Chapter 7 \\ CONCLUSION AND RECOMMENDATIONS}

\subsection{Conclusion}

Small- and medium-sized VAWTs can be utilized effectively as stand-alone wind energy generation sources if their efficiency can be further enhanced. This thesis focuses on development of a straight-bladed Darrieus Vertical Axis Wind Turbine (H-type VAWT) and increasing its performance in terms of power output and self-starting capability using an intelligent blade pitching technique, which includes selecting appropriate size of H-type VAWT as well as the pitch angles. The effects of these pitch angles on the performance of the wind turbine in terms of power output and self-starting capability were numerically investigated using Computational Fluid Dynamics (CFD) of 2D H-type VAWT model. The CFD results were used to design a blade pitch control system. The stability analysis for the proposed pitch control system was also carried out using Lyapunov theory. Finally, the outdoor field-testing of full-scale prototype of the H-type VAWT was carried out to validate the proposed design and the intelligent blade control methods developed.

The Computational Fluid Dynamics (CFD) solver was employed to examine the 2D flow physics of the H-type VAWT with an NACA0018 airfoil at different tip speed ratios (TSRs) as well as different pitch angles. The effects of varying blade pitch on both performance and self-starting capability of an H-type VAWT were also investigated. Individual blade pitching can be a powerful strategy to improve the performance of the H-type VAWT by delaying the dynamic stall. Also, the poor self-starting capability of the H-type VAWT can be enhanced at low TSRs. From CFD results, the optimum pitch angles, which maximized the power coefficients, were determined at different TSRs for one revolution and then used to design the proposed intelligent blade pitch controller for an H-type VAWT. 
A full-scaled H-type VAWT was modeled using the MatlablSimulink, which includes the proposed pitch control system. The CFD results were used for mapping the H-type VAWT rotor for both fixed and variable pitch angle cases using an LUT as well as an MLP-ANN, respectively. A novel active blade pitch control system based on MLP-ANN, which is combined with PID controller (referred to as Hybrid controller), was developed to control each blade individually. Effects of different gain contributions of both MLP-ANN and PID pitch signals on the power output of the H-type VAWT were examined.

Although the ability to track the desired pitch angle is comparable for both controllers (i.e., MLP-ANN only and Hybrid), the improvement achieved when the small gains of PID commands were added has been clearly observed in the power output curves.

The simulation and experimental results reveals that, compared to the fixed pitch angle operation, blade pitching technique clearly increased the power output of the H-type VAWT, with a percentage improvement that exceeded $25 \%$.

Moreover, the stability of the proposed closed-loop control system was investigated by using the Lyapunov theory for the H-type VAWT in both fixed and variable pitch angle configurations.

Using Lyapunov's stability theory showed that the closed loop system is asymptotically stable when the proposed blade pitch control law (i.e., Hybrid controller) is applied to the Htype VAWT model.

In addition, the system performance equations were expressed as simultaneous differential equations in state space, which are then linearized and analyzed using the Lyapunov direct method. The results showed that a positive definite $P$ matrix exists for pitch angles in two different regions of TSRs: $1<\mathrm{TSR}<1.1$ and $2.2<\mathrm{TSR}<3.3$ which means that these are the stable regions for the proposed Hybrid controller. 
For experimental validation, a full-scale of H-type VAWT prototype with blade pitching capability was built to examine the effect of blade pitching on performance of an H-type VAWT in terms of power output and self-starting capability by comparison to the fixed pitch angle configuration.

Three main outdoor tests were successfully carried out under different operating conditions: the preliminary test was conducted to examine the stability of mechanical structure for the H-type VAWT prototype and its rotor speed. The first test was performed to investigate the power output, self-starting capability, and the proposed Hybrid control effectiveness in both fixed and variable blade pitch configurations. The second test is conducted to confirm the findings of the first test.

The results obtained from the first and second tests showed that the blade pitching technique increased the power output of the H-type VAWT by about $22 \%$.

The effectiveness of the Hybrid control system was also investigated experimentally. The results showed that the use of high percentage of MLP-ANN in the Hybrid controller improved the blade pitch control response in terms of reducing overshoot at high RPMs. However, the Hybrid controller with high percentage of PID controller reduced the overshooting of pitch control response at low RPMs.

The power coefficient $\left(C_{p}\right)$ curve for the first test was obtained from the measured power output and then validated numerically by additional simulations of CFD model for a 2D Htype VAWT. Although the CFD results showed overestimation of $C_{p}$ values as was discussed in Chapter 6, there was still a good agreement between the experimental and numerical curves. 


\subsection{Recommendations}

Several recommendations can be made for more improvements of the H-type VAWT performance. For the CFD model, additional studies, such as, revisiting the mesh quality by using more mesh elements around each blade and examination of some important parameters (e.g., turbulence model, number of revolutions for steady state, 3D effect, etc.) can further improve the accuracy of the simulation results.

For experiment improvement; using composite materials can reduce the bulk weight of the VAWT especially for blades, resulting in reduction of, the total cost and expected deflection of blade linkages. Also, the transmission system design needs to be improved in terms of selection of appropriate size and material of pulleys as well as the type of belt. The vibration of the VAWT rotor in $x$ and $y$ directions on the horizontal plane can be reduced by adding a ball bearing at the top of main rotating shaft.

For the model testing, more tests outdoors in an open field are highly recommended to collect more data for further analysis and validate the H-type VAWT and the proposed Hybrid controller performance under a wide range of operating conditions.

\subsection{Future Scope}

This thesis proposed a novel control method to improve the performance of an H-type VAWT in terms of power generation and self-starting capability. An intelligent pitch control system for blade pitching is the main contribution of this thesis. This research forms the foundation for future research which can focus on:

1. For the CFD model, the effect of different solidity parameters can be investigated by changing the airfoil profiles and size of the H-type VAWT in order to enhance the design of the H-type VAWT as well as its performance. Also, a 3D CFD model should be used instead of the 2D model in order to be comparable with the real experimental model. To investigate the effect of different pitch angles on the VAWT performance, 
User Defined Functions (UDF) in the Fluent software can be considered, which can also in order to reduce the number of CFD model simulations.

2. For the pitch control system, different other controllers can be studied such as, adaptive controller and fuzzy logic based controller for the blade pitching. To further improve the pitch control response experimentally, it may be more effective if the MLP-ANN can be trained online. In addition, installing a Savonius VAWT on top of the H-Darrius wind turbine's main shaft can be investigated in order to improve the self-starting capability. 


\section{REFERENCES}

[1] N. Ion, B. Radu, and S. Marcel, "Small power wind turbine (Type DARRIEUS)," Incas Bull., vol. 4, no. 1, pp. 135-142, 2012.

[2] Z. Li-xun, L. Ying-bin, L. Xiao-hong, and G. Jian, "Effect of blade pitch angle on aerodynamic performance of straight-bladed vertical axis wind turbine," J. Cent. South Univ. Technol. (Engl. Ed.), vol. 21, pp. 1417-1427, 2014.

[3] B. Chen, S. Su, I. M. Viola, and C. A. Greated, "Numerical investigation of verticalaxis tidal turbines with sinusoidal pitching blades," Ocean Eng., vol. 155, no. January, pp. 75-87, 2018.

[4] C. Li, Y. Xiao, Y. lin Xu, Y. xin Peng, G. Hu, and S. Zhu, "Optimization of blade pitch in H-rotor vertical axis wind turbines through computational fluid dynamics simulations," Appl. Energy, vol. 212, no. January, pp. 1107-1125, 2018.

[5] R. B. Sumantraa, S. Chandramouli, T. P. Premsai, P. Prithviraj, M. Vivek, and V. R. Kishore, "Numerical Analysis of Effect of Pitch Angle on a Small Scale Vertical Axis Wind Turbine," J. Renew. Energy Res., vol. 4, no. 4, pp. 929-935, 2014.

[6] A. Sagharichi, M. Zamani, and A. Ghasemi, "Effect of solidity on the performance of variable-pitch vertical axis wind turbine,” Energy, vol. 161, pp. 753-775, 2018.

[7] J. J. Miau et al., "Design and Test of a Vertical-Axis Wind Turbine with Pitch Control," Appl. Mech. Mater., vol. 225, pp. 338-343, 2012.

[8] I. S. Hwang, S. Y. Min, I. O. Jeong, Y. H. Lee, and S. J. Kim, "Efficiency Improvement of a New Vertical Axis Wind Turbine by Individual Active Control of Blade Motion," 2004.

[9] D. W. Erickson, J. J. Wallace, and J. Peraire, "Performance Characterization of Cyclic Blade Pitch Variation on a Vertical Axis Wind Turbine," in 49th AIAA Aerospace Sciences Meeting, Including the New Horizons Forum and Aerospace Exposition, 2011, pp. 1-23.

[10] T. Ma, H. Yang, and L. Lu, "Study on stand-alone power supply options for an isolated community,” Int. J. Electr. Power Energy Syst., vol. 65, pp. 1-11, 2015.

[11] W. Peter and W. Uli, Physics of Solar Cells: From Basic Principles to Advanced Concepts, Third. John Wiley \& Sons., 2016. 
[12] Y. Chen, "Numerical Simulation of the Aerodynamic Performance of an H-Rotor .," Master's Thesis, University of Louisville, 2011.

[13] R. Howell, N. Qin, J. Edwards, and N. Durrani, "Wind tunnel and numerical study of a small vertical axis wind turbine," Renew. Energy, vol. 35, no. 2, pp. 412-422, 2010.

[14] R. Tiwari and R. B. N, "Comparative Analysis of Pitch Angle Controller Strategies for PMSG Based Wind Energy Conversion System," Int. J. Intell. Syst. Appl., vol. 9, no. 5, pp. 62-73, 2017.

[15] T. J. Carrigan, B. H. Dennis, Z. X. Han, and B. P. Wang, "Aerodynamic Shape Optimization of a Vertical-Axis Wind Turbine Using Differential Evolution," ISRN Renew. Energy, vol. 2012, pp. 1-16, 2012.

[16] S. Li, "Numerical study on the performance effect of solidity on the straight-bladed vertical axis wind turbine," in Power and energy Engineering Conference, 2010, no. 1153, pp. 1-4.

[17] Wikiwand, "Vertical axis wind turbine." [Online]. Available: http://www.wikiwand.com/en/Vertical_axis_wind_turbine.

[18] A. El Kasmi and C. Masson, "An extended model for turbulent flow through horizontal-axis wind turbines," J. Wind Eng. Ind. Aerodyn., vol. 96, no. 1, pp. 103$122,2008$.

[19] R. Bos, "Self-Starting of a Small Urban Darrieus Rotor. Strategies to Boost Performance in Low-Reynolds-Number Flows," Master's Thesis, Delft University of Technology, 2012.

[20] D. Rathi, "Performance Prediction and Dynamic Model Analysis of Vertical Axis Wind Turbine Blades with Aerodynamically Varied Blade Pitch," Master's Thesis, The Graduate Faculty of North Carolina State University, 2012.

[21] Y. Yang, Z. Guo, Y. Zhang, H. Jinyama, and Q. Li, "Numerical investigation of the tip vortex of a straight-bladed vertical axis wind turbine with double-blades," Energies, vol. 10, no. 11, 2017.

[22] E. Dyachuk and A. Goude, "Numerical validation of a vortex model against experimental data on a straight-bladed vertical axis wind turbine," Energies, vol. 8, no. 10, pp. 11800-11820, 2015. 
[23] Q. Li, T. Maeda, Y. Kamada, J. Murata, T. Kawabata, and K. Furukawa, "Analysis of Aerodynamic Load on Straight-Bladed Vertical Axis Wind Turbine," J. Therm. Sci., vol. 23, no. 4, pp. 315-324, 2014.

[24] R. Gosselin, G. Dumas, and M. Boudreau, "Parametric Study of H-Darrieus VerticalAxis Turbines Using uRANS Simulations,” Lmfn.Ulaval.Ca, vol. 178, 2013.

[25] B. K. Kirke, "Evaluation of self-starting vertical axis wind turbines for stand-alone applications.," Doctoral dissertation,Griffith University Gold Coast, 1998.

[26] J. R. Baker, "Features to aid or enable self starting of fixed pitch low solidity vertical axis wind turbines," J. Wind Eng. Ind. Aerodyn., vol. 15, no. 1-3, pp. 369-380, 1983.

[27] N. Hill, R. Dominy, G. Ingram, and J. Dominy, "Darrieus turbines: the physics of selfstarting," Proc. Inst. Mech. Eng. Part A J. Power Energy, vol. 223, no. 1, pp. 21-29, 2009.

[28] P. R. Ebert and D. H. Wood, "Observations of the Starting Behaviour of a Small Horizontal- Axis Wind Turbine," Renew. Energy, vol. 12, no. 3, pp. 1-13, 1997.

[29] C. C. Chen and C. H. Kuo, "Effects of pitch angle and blade camber on flow characteristics and performance of small-size Darrieus VAWT," J. Vis., vol. 16, no. 1, pp. 65-74, 2013.

[30] E. Dyachuk, M. Rossander, A. Goude, and H. Bernhoff, "Measurements of the Aerodynamic Normal Forces on a 12-kW Straight-Bladed Vertical Axis Wind Turbine,” Energies, vol. 8, no. 8, pp. 8482-8496, 2015.

[31] S. S. Khalid, Z. Liang, S. Qi-hu, and Z. Xue-Wei, "Difference Between Fixed and Variable Pitch Vertical Axis Tidal Turbine-Using CFD Analysis in CFX," Res. J. Appl. Sci. Eng. Technol., vol. 5, no. 1, pp. 319-325, 2013.

[32] N. C. . Pawsey, "Development and Evaluation of Passive Variable-pitch Vertical Axis Wind Turbines," Doctoral dissertation, The University of New South Wales, 2002.

[33] L. Lazauskas, "Three Pitch Control Systems for Vertical Axis Wind Turbine Compared," Wind Engineering, vol. Vol. 16, no. No. 5. pp. 269-282, 1992.

[34] P. Jain and Abhishek, "Analysis and prediction of Vertical cycloidal Rotor Wind Turbine with variable amplitude pitching," ARF 2015 - 4th Asian-Australian Rotorcr. 
Forum, pp. 5-7, 2015.

[35] I. S. Hwang, S. Y. Min, I. O. Jeong, Y. H. Lee, and S. J. Kim, "Efficiency improvement of a new vertical axis wind turbine by individual active control of blade motion," Smart Struct. Mater. 2006 Smart Struct. Integr. Syst., vol. 6173, no. April 2006, p. 617311, 2006.

[36] M. Benedict, V. Lakshminarayan, J. Pino, and I. Chopra, "Aerodynamics of a SmallScale Vertical-Axis Wind Turbine with Dynamic Blade Pitching," AIAA J., vol. 54, no. 3, pp. 924-935, 2015.

[37] C. Li, S. Zhu, Y. Xu, and Y. Xiao, “2.5D Large Eddy Simulation of Vertical Axis Wind Turbine in Consideration of High Angle of Attack Flow," Renew. Energy, vol. 51, pp. 317-330, 2013.

[38] A. Bianchini, L. Ferrari, and S. Magnani, "Start-up Behavior of a Three-Bladed HDarrieus VAWT: Experimental and Numerical Analysis," in ASME Turbo Expo:Turbine Technical Conference and Exposition, 2011, pp. 811-820.

[39] C. Nguyen, T. Le, and P. Tran, "A Numerical Study of Thickness Effect of the Symmetric NACA 4-Digit Airfoils on Self Starting Capability of a $1 \mathrm{~kW} \mathrm{H-type}$ Vertical Axis Wind Turbine," Int. J. Mech. Eng. Appl., vol. 3, pp. 7-16, 2015.

[40] I. Paraschivoiu, Wind Turbine Design: with Emphasis on Darrieus Concept. Presses inter Polytechnique, 2002.

[41] Z. Zhao et al., "Variable Pitch Approach for Performance Improving of StraightBladed VAWT at Rated Tip Speed Ratio,”Appl. Sci., vol. 8, no. 6, p. 957, 2018.

[42] P. Jain and A. Abhishek, "Performance Prediction and Fundamental Understanding of Small Scale Vertical Axis Wind Turbine with Variable Amplitude Blade Pitching," Renew. Energy, vol. 97, pp. 97-113, 2016.

[43] F. Balduzzi, A. Bianchini, R. Maleci, G. Ferrara, and L. Ferrari, "Critical Issues in the CFD Simulation of Darrieus Wind Turbines," Renew. Energy, vol. 85, pp. 419-435, 2016.

[44] M. R. Castelli, G. Ardizzon, L. Battisti, E. Benini, and G. Pavesi, "Modeling Strategy and Numerical Validation," Proc. ASME 2010 Int. Mech. Eng. Congr. Expo. IMECE2010, pp. 1-10, 2015. 
[45] F. Balduzzi, A. Bianchini, R. Maleci, G. Ferrara, and L. Ferrari, "Blade Design Criteria to Compensate the Flow Curvature Effects in H-Darrieus Wind Turbines," $J$. Turbomach., vol. 137, no. 1, p. 011006, 2014.

[46] P. Vittecoq and A. Laneville, "The aerodynamic forces for a Darrieus rotor with straight blades: Wind tunnel measurements," J. Wind Eng. Ind. Aerodyn., vol. 15, no. 1-3, pp. 381-388, 1983.

[47] R. Hantoro, I. K.A.P Utama, E. Erwandi, and A. Sulisetyono, “An Experimental Investigation of Passive Variable-Pitch Vertical-Axis Ocean Current Turbine," ITB J. Eng. Sci., vol. 43, no. 1, pp. 27-40, 2011.

[48] A. J. Fiedler and S. Tullis, "Blade Offset and Pitch Effects on a High Solidity Vertical Axis Wind Turbine," Wind Eng., vol. 33, no. 3, pp. 237-246, 2009.

[49] S. Armstrong, A. Fiedler, and S. Tullis, "Flow separation on a high Reynolds number, high solidity vertical axis wind turbine with straight and canted blades and canted blades with fences," Renew. Energy, vol. 41, pp. 13-22, 2012.

[50] T. L. Van, T. H. Nguyen, D. Lee, and S. Member, "Advanced Pitch Angle Control Based on Fuzzy Logic for Variable-Speed Wind Turbine Systems," IEEE Trans. Energy Cone, vol. 30, no. 2, pp. 578-587, 2015.

[51] S. Lee, Y. Joo, J. Back, and J. H. Seo, "Sliding Mode Controller for Torque and Pitch Control of Wind Power System Based on PMSG," in International Conference on Control, Automation and Systems, 2010, pp. 1079-1084.

[52] N. K. Kasabov, Foundations of Neural Networks, Fuzzy Systems, and Knowledge Engineering. Cambridge, Massachusetts: The MIT Press, 1998.

[53] O. Barambones, "A Robust Wind Turbine Control using a Neural Network Based Wind Speed Estimator,” in Inte. Joint Conf. on Neural Networks Ijcnn, 2010, pp. 1-8.

[54] J. Sargolzaei, "Prediction of the Power Ratio and Torque in Wind Turbine Savonius Rotors Using Artificial Neural Networks," in WSEAS International Conference on Renewable Energy Sources, 2007, vol. 1, no. 2, pp. 7-12.

[55] A. Hossain, A. Rahman, M. Rahman, S. Hasan, and J. Hossen, "Prediction of Power Generation of Small Scale Vertical Axis Wind Turbine Using Fuzzy Logic," J. Urban Environ. Eng., vol. 3, no. 2, pp. 43-51, 2009. 
[56] H. K. Versteeg and W. Malalasekera, An introduction to computational fluid dynamics: the finite volume method. Pearson Education, 2007.

[57] R. Bhaskaran and L. Collins, "Introduction to CFD basics," Cornell University-Sibley School of Mechanical and Aerospace Engineering, pp. 1-21, 2002.

[58] Ansys, "Meshing Help,” 2009.

[59] M. Chmielewski and M. Gieras, "Three-zonal Wall Function for k- $\varepsilon$ Turbulence Models," Comput. Methods Sci. Technol., vol. 19, no. 2, pp. 107-114, 2013.

[60] S. Florian R. Menter, Eloret Insitute, "Zonal Two Equation k-Omega, Turbulence Models for Aerodynamic Flows," AIAA-Journal, 1994.

[61] Fluent, “ANSYS® Fluent User Guide, Release 15.0,” SAS IP, Inc, 2013.

[62] L. X. Zhang, Y. B. Liang, X. H. Liu, Q. F. Jiao, and J. Guo, "Aerodynamic performance prediction of straight-bladed vertical axis wind turbine based on CFD," Adv. Mech. Eng., vol. 5, 2013.

[63] M. Singh, "Dynamic Models for Wind Turbines and Wind Power Plants," Natl. Renew. Energy Lab., vol. 1, no. May, p. 115, 2011.

[64] A. Rolán, Á. Luna, J. Rocabert, D. Aguilar, and G. Vázquez, “An Approach to the Performance-Oriented Model of Variable-Speed Wind Turbines," in IEEE International Symposium on Industrial Electronics, 2010, pp. 3853-3858.

[65] X. Zhang, D. Xu, and Y. Liu, "Intelligent Control for Large-Scale Variable Speed," J. Control Theory Appl., vol. 3, no. December 2003, pp. 305-311, 2004.

[66] H. Wagner and J. Mathur, Introduction to Wind Energy Systems. Springer Science \& Business Media, 2009.

[67] C. N. Bhende, S. Mishra, and S. G. Malla, "Permanent magnet synchronous generatorbased standalone wind energy supply system," IEEE Trans. Sustain. Energy, vol. 2, no. 4, pp. 361-373, 2011.

[68] S. A. Khan, R. K. Rajkumar, R. K. Rajkumar, and A. Cv, "Performance analysis of 20 Pole 1.5 KW Three Phase Permanent Magnet Synchronous Generator for low Speed Vertical Axis Wind Turbine,” Energy Power Eng., vol. 05, no. 04, pp. 423-428, 2013.

[69] S. Mohanty, "Artificial Neural Network Based System Identification and Model 
Predictive Control of a Flotation Column,” J. Process Control, vol. 19, no. 6, pp. 991999, 2009.

[70] A. Seyab, R. K. Shakir, and Y. Cao, "Nonlinear system identification for predictive control using continuous time recurrent neural networks and automatic differentiation.," J. Process Control, vol. 18, no. 6, pp. 568-581, 2008.

[71] K. Funahashi and Y. Nakamura, "Approximation of dynamical systems by continuous time recurrent neural networks," Neural Networks, vol. 6, no. 6, pp. 801-806, 1993.

[72] F. O. Karray and C. De Silva, Soft Computing and Intelligent Systems Design: Theory, Tools, and Applications. Pearson Education Limited, 2004.

[73] S. M. Muyeen et al., "Comparative study on transient stability analysis of wind turbine generator system using different drive train models," IET Renewable Power Generation, vol. 1, no. 2. p. 131, 2007.

[74] P. Badoni and S. B. Prakash, "Modeling and Simulation of 2 MW PMSG Wind Energy Conversion Systems," IOSR J. Electr. Electron. Eng., vol. 9, no. 4, pp. 53-58, 2014.

[75] Y. Vidal, L. Acho, N. Luo, M. Zapateiro, and F. Pozo, "Power control design for variable-speedwind turbines," Energies, vol. 5, no. 8, pp. 3033-3050, 2012.

[76] L. A. K. \&P. S. S. Sumathi, Solar PV and Wind Energy Conversion Systems. ,eBook, Springer International Publishing, 2015.

[77] P. B. Thakkar, "Neural Networks Satisfying Stone-Weiestrass Theorem and Approximating Scattered Data by Kohonen Neural Networks," Master's Thesis, University of Central Florida, 2004.

[78] M. Lee, G. Hwang, W. Jang, and K. Cha, "Robotic Agent Control Based on Adaptive Intelligent," Springer-Verlag Berlin Heidelb., pp. 539-548, 2007.

[79] S. Pezeshki, S. Badalkhani, and A. Javadi, "Performance Analysis of a Neuro-PID Controller Applied to a Robot Manipulator," Int. J. Adv. Robot. Syst., vol. 9, pp. 1-10, 2012.

[80] J. Aho, A. Buckspan, and J. H. Laks, "A tutorial of wind turbine control for supporting grid frequency through active power control," Proc. Am. Control Conf., no. March, pp. 
$3120-3131,2012$.

[81] S. Sapna, A. Tamilarasi, and M. Pravin Kumar, "Backpropagation Learning Algorithm Based on Levenberg Marquardt Algorithm," Comp Sci Inf. Technol (CS IT), vol. 2, pp. 393-398, 2012.

[82] L. A. Danao, "The Influence of Unsteady Wind on the Performance and Aerodynamics of Vertical Axis Wind Turbines," Doctoral dissertation, The University of Sheffield, 2012.

[83]

“SolidWorks," Wikipedia, 2016. [Online]. Available: http://en.wikipedia.org/wiki/SolidWorks.

[84] B. Roscher, "Structural Optimization Of A Vertical Axis Wind Turbine With Aeroelastic Analysis," M.Sc. Thesis - Delft Univ. Technol., no. June, 2014.

[85] G. Semrau and G. Semrau, "Dynamic modeling and characterization of a wind turbine system leading to controls development," Master's thesis, Rochester Institute of Technology, 2010.

[86] S. C. Thomsen, "Nonlinear Control of a Wind Turbine," Master's thesis; the Technical University of Denmark (DTU), 2006.

[87] G. Semrau, S. Rimkus, and T. Das, "Nonlinear Systems Analysis and Control of Variable Speed Wind Turbines for Multiregime Operation," J. Dyn. Syst. Meas. Control, vol. 137, no. 4, p. 041007, 2015.

[88] K. E. Johnson, L. Y. Pao, M. J. Balas, and L. E. E. J. Fingersh, “Control of VariableSpeed Wind Turbines," IEEE Control Syst. Mag., vol. 26(3), no. June, pp. 70-81, 2006.

[89] E. B. Muhando, T. Senjyu, A. Uehara, T. Funabashi, and C. H. Kim, "LQG design for megawatt-class WECS with DFIG based on functional models' fidelity prerequisites," IEEE Trans. Energy Convers., vol. 24, no. 4, pp. 893-904, 2009.

[90] X. Jiao, W. Meng, Q. Yang, L. Fu, and Q. Chen, “Adaptive Continuous Neural Pitch Angle Control for Variable-Speed Wind Turbines," Asian J. Control, vol. 21, no. 2, pp. 1-14, 2018.

[91] H. K. Khalil, Nonlinear Systems. Macmillan Publishing Company, 1992. 
[92] R. M. Murray, Z. Li, and S. S. Sastry, A Mathematical Introduction to Robotic Manipulation. CRC Press, 1994.

[93] A. D. O. D. S. Dantas, A. F. O. D. A. Dantas, J. T. L. S. Campos, D. L. De Almeida Neto, and C. E. T. Dórea, "PID Control for Electric Vehicles Subject to Control and Speed Signal Constraints," J. Control Sci. Eng., vol. 2018, 2018.

[94] "Rectification of a Three Phase Supply using Diodes." [Online]. Available: https://www.electronics-tutorials.ws/power/three-phase-rectification.html.

[95] U. of Waterloo, "UW Weather Station." [Online]. Available: http://weather.uwaterloo.ca/.

[96] Chambers, John, W. Cleveland, B. Kleiner, and P. Tukey, Graphical Methods for Data Analysis, vol. 9, no. 7. 1983.

[97] M. MathWorks, "Wind Turbine," 2019. [Online]. Available: https://www.mathworks.com/help/physmod/sps/powersys/ref/windturbine.html.

[98] D. Sayeef, Saad; Heslop, Simon; Cornforth, David; Moore, Tim; Percy, Steven; Ward, John; Berry, Adam; Rowe, "Solar intermittency: Australia's clean energy challenge. Characterising the effect of high penetration solar intermittency on Australian electricity networks," no. June, p. 195, 2012.

[99] F. Castellani, M. Mana, and D. Astolfi, "An experimental analysis of wind and power fluctuations through time-resolved data of full scale wind turbines," J. Phys. Conf. Ser., vol. 1037, no. 7, 2018.

[100] A. Efrat, L. J. Guibas, S. Har-Peled, J. S. B. Mitchell, and T. M. Murali, "New similarity measures between polylines with applications to morphing and polygon sweeping,” Discret. Comput. Geom., vol. 28, no. 4, pp. 535-569, 2002.

[101] A. Cimpoeru, "Encoderless Vector Control of PMSG for Wind Turbine Applications," Master's Thesis, Aalborg University, 2010.

[102] B. D. S. Rao, C. H. Kumar, and V. S. Babu, "Power Flow in PMSG Connected Wind Energy Conversion System through Current Source Converter Using Space Vector Modulation Technique," Int. J. Adv. Comput. Res., vol. 3, no. 11, pp. 70-75, 2013.

[103] C. N. Wang, W. C. Lin, and X. K. Le, "Modelling of a PMSG wind turbine with 
autonomous control," Math. Probl. Eng., vol. 2014, p. 9, 2014.

[104] A. Bharathi and R. Seyezhai, "MATLAB Simulation of Power Electronic Converter for PMSG Based Wind Energy Conversion System," Int. J. Innov. Res. Electr. Electron. Instrum. Control Eng., vol. 1, no. 8, pp. 348-353, 2013.

[105] A. S. Yilmaz and Z. Özer, "Pitch Angle Control in Wind Turbines above the Rated Wind Speed by Multi-layer Perceptron and Radial Basis Function Neural Networks," Expert Syst. Appl., vol. 36, no. 6, pp. 9767-9775, 2009.

[106] hadi kasiri, H. Reza, and A. Kasiri, “Optimal Intelligent Control for Wind Turbulence Rejection in WECS Using ANNs and Genetic Fuzzy Approach,” Int. J. Soft Comput. Softw. Eng., vol. 2, no. 9, pp. 16-34, 2012.

[107] S. H. Oh, "Error Back-Propagation Algorithm for Classification of Imbalanced Data," Neurocomputing, vol. 74, no. 6, pp. 1058-1061, 2011.

[108] B. Raton, L. New, and F. Group, Nonlinear systems stability analysis: Lyapunov-based approach. CRC Press, 2013.

[109] M. Smolik and V. Skala, "Vector Field Interpolation with Radial Basis Functions," Proc. Sigr. 2016, May 23rd 24th, Visby, Sweden, no. 127, pp. 15-21, 2016.

[110] G. Abdalrahman, W. Melek, and F. S. Lien, "Pitch angle control for a small-scale Darrieus vertical axis wind turbine with straight blades (H-Type VAWT)," Renew. Energy, vol. 114, pp. 1353-1362, 2017. 


\section{APPENDICES}

\section{Appendix A}

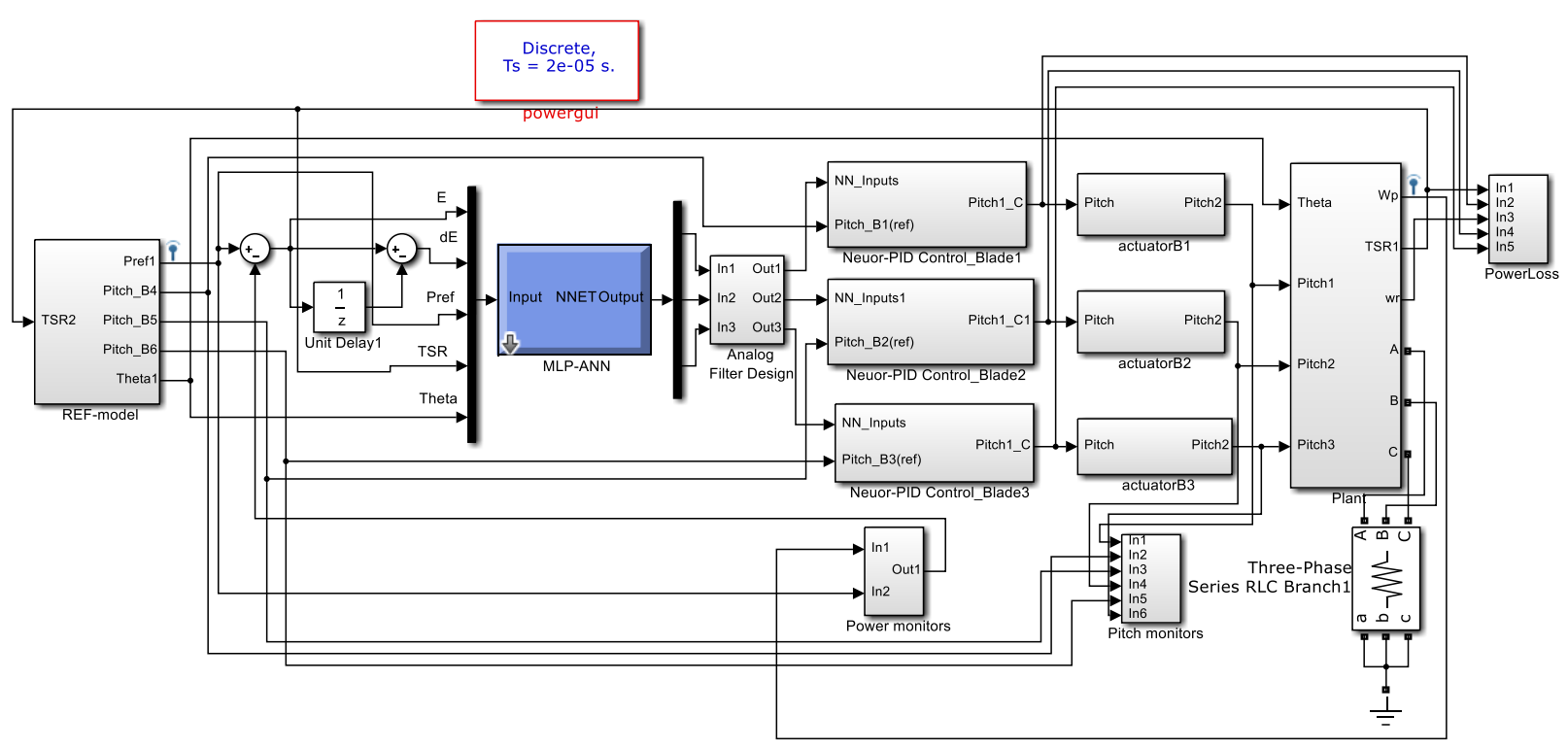

Figure A1. The H-type VAWT Matlab/Simulink model.

Table A1. The parameters of 2-mass drive train model.

\begin{tabular}{lc}
\hline \multicolumn{1}{c}{ Parameter Name } & Value \\
\hline Inertia constant $(H)[\mathrm{sec}]$ & 2 \\
Stiffness $\left(k_{s}\right)[\mathrm{pu} / \mathrm{rad}]$ & 0.1 \\
Damping $\left(D_{s}\right)[\mathrm{pu} \cdot \mathrm{sec} / \mathrm{rad}]$ & 1 \\
\hline
\end{tabular}

Table A2. The PMSG design parameters.

\begin{tabular}{lc}
\hline \multicolumn{1}{c}{ Parameter Name } & Value \\
\hline Stator Phase Resistance $\left(\mathrm{R}_{\mathrm{s}}\right)[\mathrm{ohm}]$ & 0.425 \\
Inductance $(\mathrm{d}, \mathrm{q})\left(L_{d}=L_{q}\right)[\mathrm{mH}]$ & 0.835 \\
Flux Linkage $(\varphi)[\mathrm{V} \cdot \mathrm{s}]$ & 0.433 \\
Inertia $\left[\mathrm{kg} \cdot \mathrm{m}^{2}\right]$ & 0.01197 \\
Pole Pair $(p)$ & 5 \\
Rated Power $[\mathrm{KW}]$ & 4 \\
Nominal Frequency $[\mathrm{Hz}]$ & 50 \\
\hline
\end{tabular}




\section{A1: PMSG model}

The equivalent circuit of PMSG based on the d-q synchronous reference frame is shown in Fig. A2. The q-axis is $90^{\circ}$ ahead of the d-axis with respect to the direction of rotation as shown in Fig. A3. It means that the d-axis is aligned with the rotor and flux while the q-axis is the perpendicular to the daxis [101].

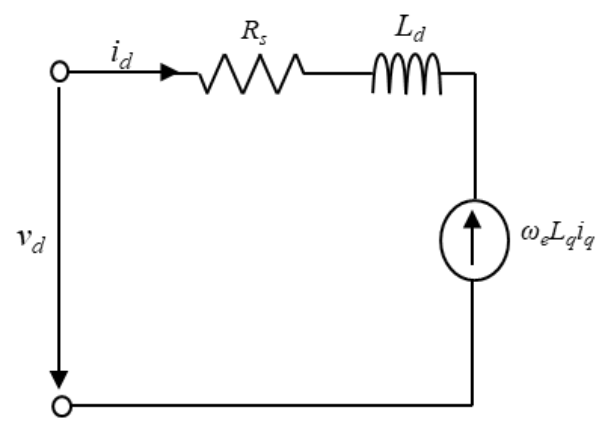

(a)

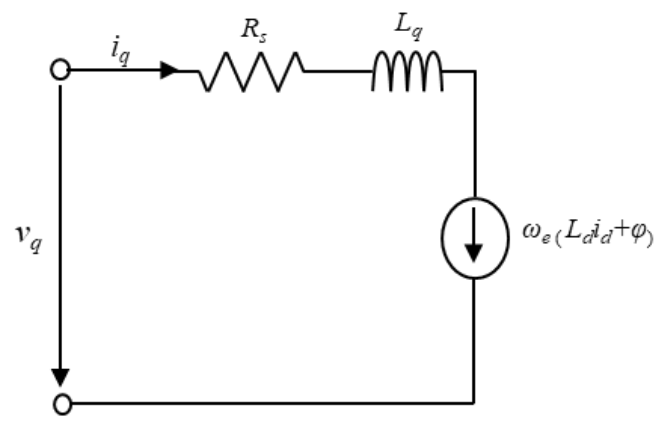

(b)

Figure A2. Equivalent circuit of the PMSG synchronous frame.

(a) d-axis circuit, (b) q-axis circuit [102]

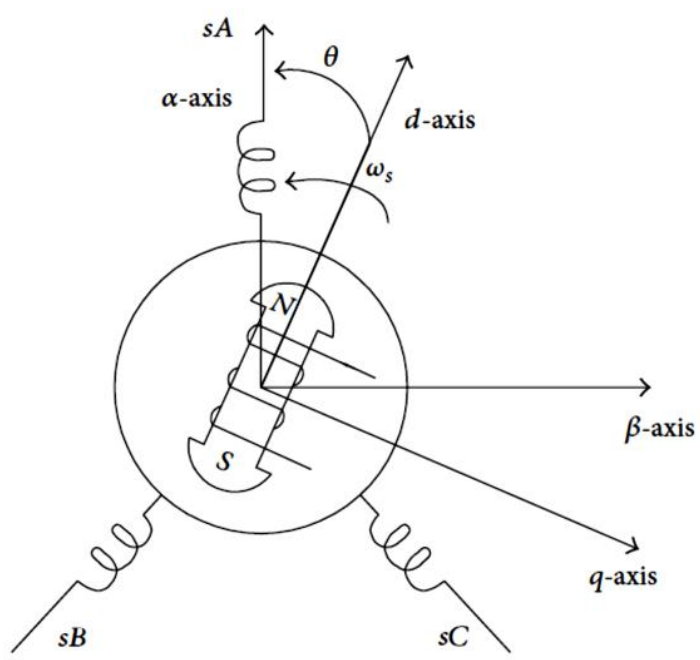

Figure A3. The $d q$-coordinate frame of the PMSG [103].

Moreover, the voltage equations of the PMSG can be expressed as follows [104]:

$$
\begin{gathered}
v_{d}=L_{d} \frac{d}{d t} i_{d}+R_{s} i_{d}-\omega_{e} L_{q} i_{q} \\
v_{q}=L_{q} \frac{d}{d t} i_{q}+R_{s} i_{q}+\omega_{e}\left(L_{d} i_{d}+\varphi\right)
\end{gathered}
$$


Where $\omega_{\mathrm{e}}$ is angular velocity of the generator, defined by,

$$
\omega_{e}=p \omega_{r}
$$

The electromagnetic torque equation is given by

$$
\tau_{e}=1.5 p\left[\varphi i_{q}+\left(L_{d}-L_{q}\right) i_{d} i_{q}\right]
$$

where $L_{q}$ is the q-axis inductance; $L_{d}$ is the d-axis inductance; $R_{s}$ is the resistance of the stator windings; $i_{q}$ is the q-axis current; $i_{d}$ is the d-axis current; $v_{q}$ is the q-axis voltage; $v_{d}$ is the d-axis voltage; $\omega_{r}$ is the angular velocity of the rotor; $\varphi$ is the amplitude of flux induced and $p$ is the number of pole pairs.

\section{A2: Structure of Multi-Layer Perceptron Artificial Neural Networks (MLP-ANN)}

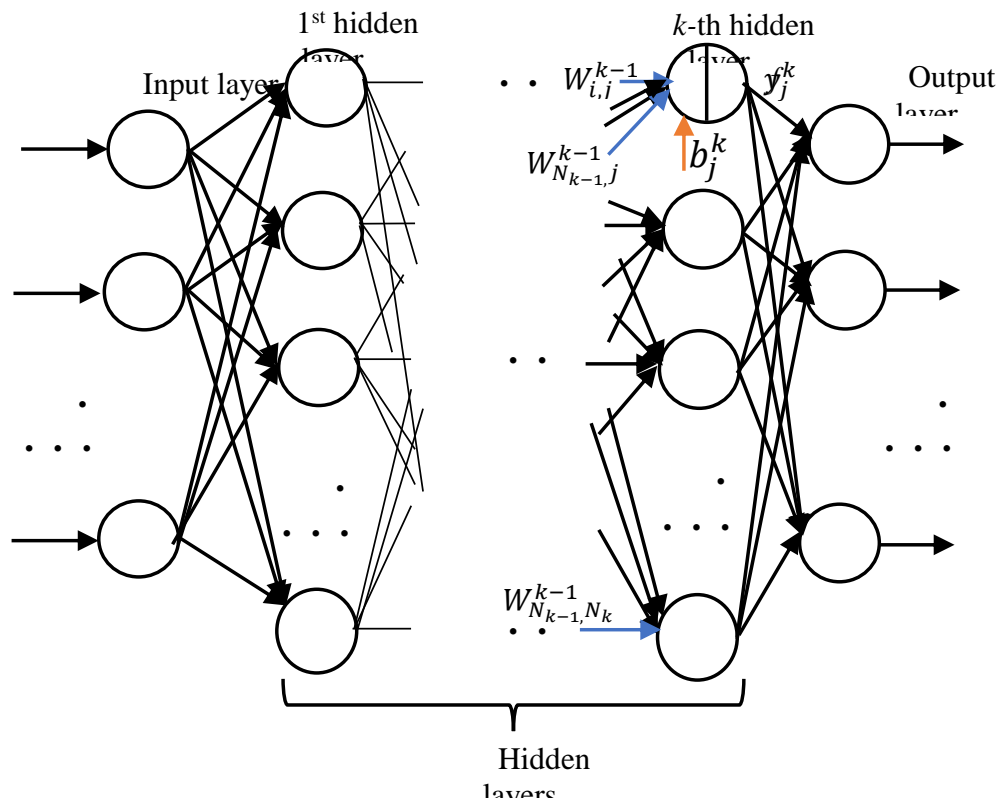

Figure A4. MLP-NN neural network structure [105]. 
Figure A4 illustrates the structure of MLP-ANN network with different layers. It is assumed that the $(k-1)$ layer has $\mathrm{N}_{k-l}$ neurons, so the input-output equation given in [105][106] is

$$
y_{j}^{(k)}=F_{j}^{(k)}\left[\sum_{i=1}^{N_{k-1}} W_{i j}^{(k-1)} y_{i}^{(k-1)}+b_{j}^{(k)}\right] ;\left(j=1,2, \ldots, N_{k} ; k=1,2, \ldots, M\right)
$$

where $W_{i j}^{(k-1)}$ is the connection weight between the $i$-th neuron in the $(k-1)$ layer to the $j$-th neuron in the $k$-th layer, $y_{j}^{(k)}$ the output of the $j$-th neuron in the $k$-th layer, $F_{j}^{(k)}$ is the activation function of the $j$-th neuron in the $k$-th layer, and $b_{j}^{(k)}$ denotes the integer bias of the of the $j$-th neuron at the $k$-th layer. In particular, the learning algorithms such as the error back-propagation algorithm (BP) have been applied widely in much scientific research. In addition, the weights are updated by the amount of error between the network output and desired output. The back-propagation (BP) learning algorithm is adopted in this research.

Eq. A6 is used for updating the weight as

$$
W_{i j}^{(k-1)}(t+1)=W_{i j}^{(k-1)}(t)+\alpha_{l} \sum_{n=1}^{I} \delta_{n j}^{(k)} y_{n i}^{(k-1)}
$$

where $t$ is the number of iterations and $\alpha_{l}$ is the learning rate and

$$
\delta_{n j}^{(k)}=\operatorname{sgm}_{n j}^{(k)}(\cdot) \cdot\left[\sum_{l=1}^{N_{k+1}} \delta_{n I}^{(k+1)} W_{j l}^{(k)}(t)\right]
$$

Weights in the back propagation algorithm [52] [107] were updated according to the errors between the network output and desired output. The square error can be calculated by

$$
\varepsilon=\sum_{n=1}^{l} \sum_{j=1}^{N_{M}}\left(y_{n j}^{(M)}-\hat{y}_{n j}^{(M)}\right)^{2}
$$

Where $y_{n j}^{(M)}$ is a target output, $\hat{y}_{n j}^{(M)}$ is an actual output. 


\section{A.2.1 MLP-ANN Matlab code}

$\%$ MLP-ANN

$\%$ Multilayer Perceptron (MLP) Neural Network Function using MATLAB

$\%$ This code is implemented for predicting pitch commands

$\%$ (MLP-ANN controller) and power coefficients (Mapping)

$\% * * * * * * * * * * * * * * * * * * * * * *$ main $\operatorname{code} * * * * * * * * * * * * * * * * * * * * *$

clear all

close all

$\mathrm{clc}$

$\%$------- load data -------

load('Data-file');

inputN =Data-file(:, $\left.{ }^{*}\right) ; \%$ \%input

target $\mathrm{N}=$ Data-file $\left(:,{ }^{*}\right) ; \%$ targets;

$\%$------- Network creation -------

hiddenLayerSize $=20$;

net $=$ patternnet $($ hiddenLayerSize $)$;

$\%$------- Initializing -------

net.trainParam.min_grad $=0.000001$;

net.trainParam.epochs $=1000 ; \%$ number of epochs

net.divideFen = 'dividerand'; \% Divide data randomly

net.divideMode = 'sample'; \% Divide up every sample

net.divideParam.trainRatio $=75 / 100 ; \%$ for training

net.divideParam.valRatio $=15 / 100 ; \%$ for validation

net.divideParam.testRatio $=25 / 100 ; \%$ for testing

net.trainParam.max_fail $=15$;

net.layers $\{1\}$.transferFen ='tansig'; \% 'logsig' activation function

net.trainFcn = 'trainlm'; \% Levenberg-Marquardt training function

net.performFcn = 'mse'; \% Mean squared error

$\%$------- Training -------

net $=$ train (net, inputN', targetN');

$\mathrm{yN}=\operatorname{net}($ inputN');

errors $=$ gsubtract $($ targetN',yN $)$;

performance $=$ perform $($ net, targetN', $\mathrm{yN})$

$\%$ figure, plotconfusion(target $\mathrm{N}^{\prime}, \mathrm{yN}$ );

gensim(net); \% generate simulink block

view(net) \% view the structure of MLP 
$\%$------ plot results--------

figure

$\operatorname{plot}\left(\mathrm{yN}, \mathrm{r}^{\prime}\right)$; hold on;

plot(targetN,'k')

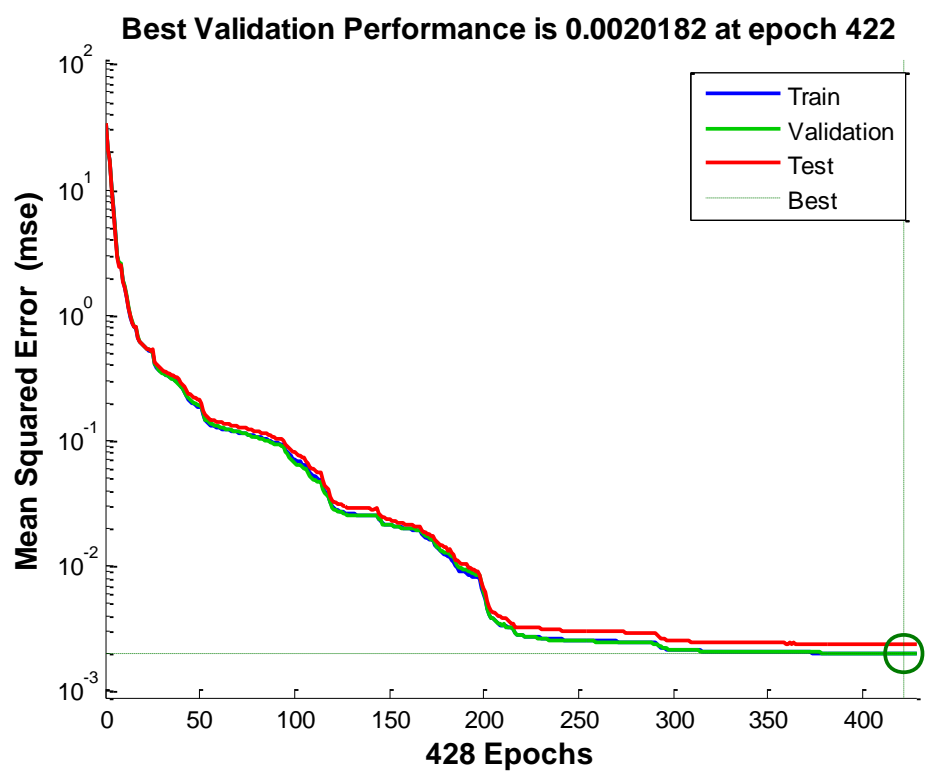

Figure A5. The performance of the MLP-ANN model during the training process. 


\section{Appendix B}

\section{B1: Lyapunov stability}

Consider a dynamical system which satisfies

$$
\dot{x}=f(x)
$$

Suppose $\bar{x} \in \mathbb{R}^{n}$ is an equilibrium point of (B1) if $f(\bar{x}) \equiv 0$ (i.e., the equilibrium point is at the origin of $\mathbb{R}^{n}$ ). This equilibrium point is locally stable if all solutions starting at nearby $\bar{x}$ (meaning that the initial conditions are in a neighborhood of $\bar{x}$ ); otherwise it is unstable. It is a locally asymptotically stable if all solutions starting at nearby points not only stay nearby, but also tend to the equilibrium point as time approaches infinity $t \rightarrow \infty$. There is no loss generality if equilibrium point is not at the origin $(\bar{x} \neq 0)$ because any equilibrium point can be shifted to the origin by means of change of variable (i.e., $y=x-\bar{x}$ ) [91].

In fact, the behavior of a nonlinear system can be described locally by the characteristics of a linear system. The method of linearizing a nonlinear plant around a chosen operating point is often denoted Lyapunov's linearization method. An order phase portraits method can be also used to examine stability [86]. Another approach which applies to systems of arbitrary order is Lyapunov's direct method which will be explained in the next section.

\section{- Lyapunov's direct method}

Lyapunov's direct method provides a number of theorems for establishing local or global stability of systems. To proceed with these the concept of a Lyapunov function need to be presented [91];

Definition 1 (Lyapunov function) Let $B_{\epsilon}$ be a ball of size $\epsilon$ around the origin. If, in a ball $B_{\epsilon}$, the function $V(x)$ is positive definite and has continuous partial derivatives, and if its time derivative along any state trajectory of the system $\dot{x}=f(x)$ is negative semi-definite. Then $V(x)$ is said to be a Lyapunov function for the system.

Theorem $\boldsymbol{i}$ (Local stability) An equilibrium state exhibits local stability if

- $V(x)$ is positive definite locally in $B_{\epsilon}$

- $\dot{V}(x)$ is negative semidefinite locally in $B_{\epsilon}$

The equilibrium is an asymptotically stable if $\dot{V}(x)$ is a negative definite. 
Theorem $\ddot{i i}$ (Global stability) For the equilibrium state to hold global (asymptotic) stability the ball $B_{\epsilon}$ must include the whole state-space. Furthermore, $V(x)$ must be radially unbounded.

- $V(x)$ is a positive definite.

- $\dot{V}(x)$ is a negative definite.

$\cdot V(x) \rightarrow \infty$ as $\|x\| \rightarrow \infty$.

The above theorems provide a conceptually simple method for analyzing a system for stability. The drawback is however that there generally is no systematic method for finding such a Lyapunov function $V(x)$. Furthermore, the theorems do not provide any information about the instability of an equilibrium point [108]. Nevertheless, the Lyapunov function can be created for stable linear systems in a systematic way. For example, a simple quadratic form for the Lyapunov function is chosen:

$$
V(x)=x^{T} P x, P=P^{T}>0
$$

The directional derivative of $V(x)$ is

$$
\begin{gathered}
\dot{V}(x)=x^{T} P \dot{x}+\dot{x}^{T} P x \\
=x^{T}\left(A^{T} P+P A\right) x \\
=-x^{T} Q x
\end{gathered}
$$

Choosing $\mathrm{P}$ as an arbitrary positive definite matrix does not generally lead to conclusive results. On the other hand, it can be shown that choosing $\mathrm{Q}$ as a symmetric positive definite matrix (which means that $\dot{V}(x)$ is negative definite) and solving for $P$ will lead to a Lyapunov function.

The equation

$$
A^{T} P+P A=-Q
$$

is the Lyapunov equation. The quadratic Lyapunov function usually is not guaranteed to determine the stability of nonlinear system. It is, however, a good starting point for stability analysis[91].

In fact, there are no given procedures to select a suitable Lyapunov function for a given dynamic system. However, the stability property of the system can be predicted by using the actual energy function of the system. In particular, the Lyapunov function can be found by replacing the energy-like function with the actual energy function in stability analysis of a dynamic system [108]. 


\section{B2: Polynomial function for power coefficient}

$$
\begin{aligned}
& C_{p}(\lambda, \beta)=C_{p}\left(x_{1}, x_{2}\right)=p_{00}+p_{10} x_{2}+p_{01} x_{1}+p_{20} x_{2}^{2}+p_{11} x_{2} x_{1}+p_{02} x_{1}^{2}+p_{30} x_{2}^{3}+ \\
& p_{21} x_{2}^{2} x_{1}+p_{12} x_{2} x_{1}^{2}+p_{03} x_{1}^{3}+p_{40} x_{2}^{4}+p_{31} x_{2}^{3} x_{1}+p_{22} x_{2}^{2} x_{1}^{2}+p_{13} x_{2} x_{1}^{3}+p_{04} x_{1}^{4}+p_{50} x_{2}^{5}+ \\
& p_{41} x_{2}^{4} x_{1}+p_{32} x_{2}^{3} x_{1}^{2}+p_{23} x_{2}^{2} x_{1}^{3}+p_{14} x_{2} x_{1}^{4}
\end{aligned}
$$

Where $p 00, p 10, p 01, p 20, p 11, p 02, p 30, p 21, p 12, p 03, p 40, p 31, p 22, p 04, p 50, p 41, p 32, p 23$, and $p 14$ are parameters that are different for each Cp curve.

\section{$\% \% \% \%$ Main code for stability analysis $\% \% \% \%$}

clear all

clc

syms x y s1 s2

$$
\begin{aligned}
& C_{p}(\lambda, \beta)=\left(\mathrm{p} 00+\mathrm{p} 10^{*} \mathrm{x}+\mathrm{p} 01 * \mathrm{y}+\mathrm{p} 20^{*} \mathrm{x} .{ }^{\wedge} 2+\mathrm{p} 11^{*} \mathrm{x} * \mathrm{y}+\mathrm{p} 02 * \mathrm{y} \cdot{ }^{\wedge} 2+\mathrm{p} 30 * \mathrm{x} \cdot{ }^{\wedge} 3+\mathrm{p} 21 * \mathrm{x} .{ }^{\wedge} 2 * \mathrm{y}\right. \\
& +\mathrm{p} 12 * \mathrm{x}^{*} \mathrm{y} .{ }^{\wedge} 2+\mathrm{p} 03^{*} \mathrm{y} .{ }^{\wedge} 3+\mathrm{p} 40^{*} \mathrm{x} .{ }^{\wedge} 4+\mathrm{p} 31^{*} \mathrm{x} .{ }^{\wedge} 3 * \mathrm{y}+\mathrm{p} 22^{*} \mathrm{x} \cdot{ }^{\wedge} 2 * \mathrm{y} .{ }^{\wedge} 2 \ldots \\
& + \text { p13*x*y.^3 + p04*y.^4 + p50*x.^5 + p41*x.^4*y + p32*x.^3*y.^2 .. } \\
& + \text { p } 23 * x \cdot{ }^{\wedge} 2 * y . \wedge 3+\text { p14*x*y.^4) } \% \% \% \text { Power coefficient }
\end{aligned}
$$

$\mathrm{x} 1=\left(\left(1.225 * 2 * 0.85^{*} 10 .^{\wedge} 3\right) /(2 * 100)\right) *(0.85 /(10 * \mathrm{y})) * \mathrm{Cp}-((15 * 20) / 100)$;

$\mathrm{A} 1=-\mathrm{a} /(1+\exp (-(11.76 * \mathrm{y}-\mathrm{b}) * \mathrm{c}))$;

$\mathrm{B}=-\mathrm{d} /\left(1+\exp \left(-\left(11.76^{*} \mathrm{y}-\mathrm{b}\right) * \mathrm{e}\right)\right)$;

$\mathrm{PID}=\mathrm{h} *(\mathrm{z}-\mathrm{x})+\mathrm{m} *(\operatorname{diff}(\mathrm{z}-\mathrm{x}))+\mathrm{k} *(\operatorname{int}(\mathrm{z}-\mathrm{x}))$

$\mathrm{x} 2=(1 / 0.15) *((1 /(1+\exp (\mathrm{A} 1+\mathrm{B})))-\mathrm{x})+\mathrm{PID}$;

$\mathrm{f} 1=\operatorname{diff}(\mathrm{x} 1, \mathrm{u})$;

$\mathrm{f} 2=\operatorname{diff}(\mathrm{x} 1, \mathrm{z} 1)$;

$\mathrm{f} 3=\operatorname{diff}(\mathrm{x} 2, \mathrm{u})$;

$\mathrm{f} 4=\operatorname{diff}(\mathrm{x} 2, \mathrm{z} 1)$;

$\mathrm{x}=\mathrm{x}(1)$;

$\mathrm{y}=\mathrm{x}(2)$;

fun=@(x)[x1;x2];\%\%\% solve $\mathrm{x} 1$ and $\mathrm{x} 2$

$\mathrm{x} 0=[1.5,6] ; \%$ intials

options = optimoptions('Isqnonlin','Algorithm','levenberg-marquardt','Display','iter');

[x,resnorm,residual,exitflag,output] =lsqnonlin(fun, 0 0,[],[],options);

$\mathrm{S}=$ lsqnonlin(fun, $\mathrm{x} 0,[],[]$, options);

quiver(W,Z,S(:,1),S(:,2),'r'); figure(gcf) \%\%\%\% phase diagram

$\mathrm{r}=\operatorname{double}(\mathrm{S})$;

$\mathrm{r}=\mathrm{r}^{\prime}$;

$\mathrm{x}=\mathrm{s} 1+\mathrm{r}(1,1)$;

$\mathrm{y}=\mathrm{s} 2+\mathrm{r}(2,1)$;

$\mathrm{x} 1=\left(\left(1.225^{*} 2 * 0.85^{*} 10 .^{\wedge} 3\right) /(2 * 100)\right) *(0.85 /(10 * \mathrm{y})) * \mathrm{Cp}-\left(\left(15^{*} 20\right) / 100\right)$;

$\mathrm{x} 2=(1 / 0.15) *((1 /(1+\exp (\mathrm{A} 1+\mathrm{B})))-\mathrm{x})+\mathrm{PID}$;

$\mathrm{s} 1=0$;

$\mathrm{s} 2=0$;

$A=\operatorname{eval}([\mathrm{f} 1 \mathrm{f} 2 ; \mathrm{f} 3 \mathrm{f} 4])$;

$\mathrm{g}=\operatorname{eig}(\mathrm{A})$

$\mathrm{Q}=\left[\begin{array}{lll}1 & 0 ; 0 & 1\end{array}\right]$;

$\% \% \% \%$ Lyap(A,Q) \%\%\%\%

$\% \mathrm{AX}+\mathrm{XA}^{\prime}+\mathrm{Q}=0$

$\% \mathrm{~A}=$ Matrix $\mathrm{A}, \mathrm{Q}=$ Matrix $\mathrm{Q}, \mathrm{X}=$ Matrix $\mathrm{X}$ 
$\%$

MatrixX = lyap(A',Q);

disp('Lyapunov Solution is MatrixX = ');

$\operatorname{disp}($ MatrixX);

$\mathrm{k}=\operatorname{eig}($ MatrixX);

disp('Eigen values of Lyapunov: ');

$\operatorname{disp}(\mathrm{k})$;

if $(\mathrm{k}(1)>0 \& \& \mathrm{k}(2)>0)$

disp('The System is Positive Definite and hence stable');

else

disp('The System is not Positive Definite and hence unstable');

end

\section{B3: Classification of critical point}

The stability can be informed based on the real part $(R e)$ of eigenvalues $(a)$ as follows [109]:

- Sink or attracting (stable node): if $\operatorname{Re}\left(a_{1}\right)<0$ and $\operatorname{Re}\left(a_{2}\right)<0$

- $\quad$ Source (unstable node): if $\operatorname{Re}\left(a_{1}\right)>0$ and $\operatorname{Re}\left(a_{2}\right)>0$

- Saddle (unstable): if $\operatorname{Re}\left(a_{1}\right)<0$ and $\operatorname{Re}\left(a_{2}\right)>0$

- Spiral or vortex: $a_{1}$ and $a_{2}$ are complex conjugates: if $\operatorname{Re}\left(a_{1}\right)<0$ then stable, if $\operatorname{Re}\left(a_{1}\right)>$ 0 then unstable. 
Appendix C

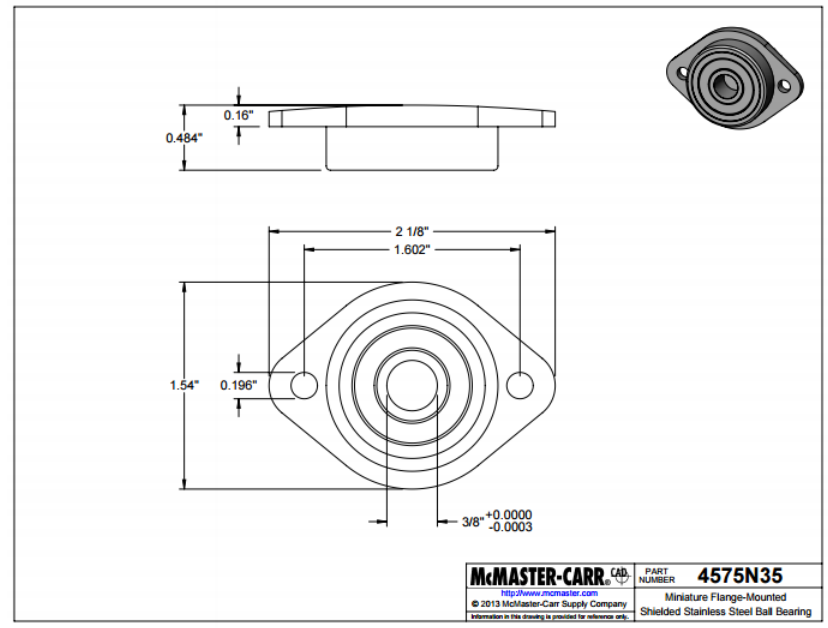

Figure $\mathrm{C} 1.3 / 8$ " bearings for top and bottom blade parts
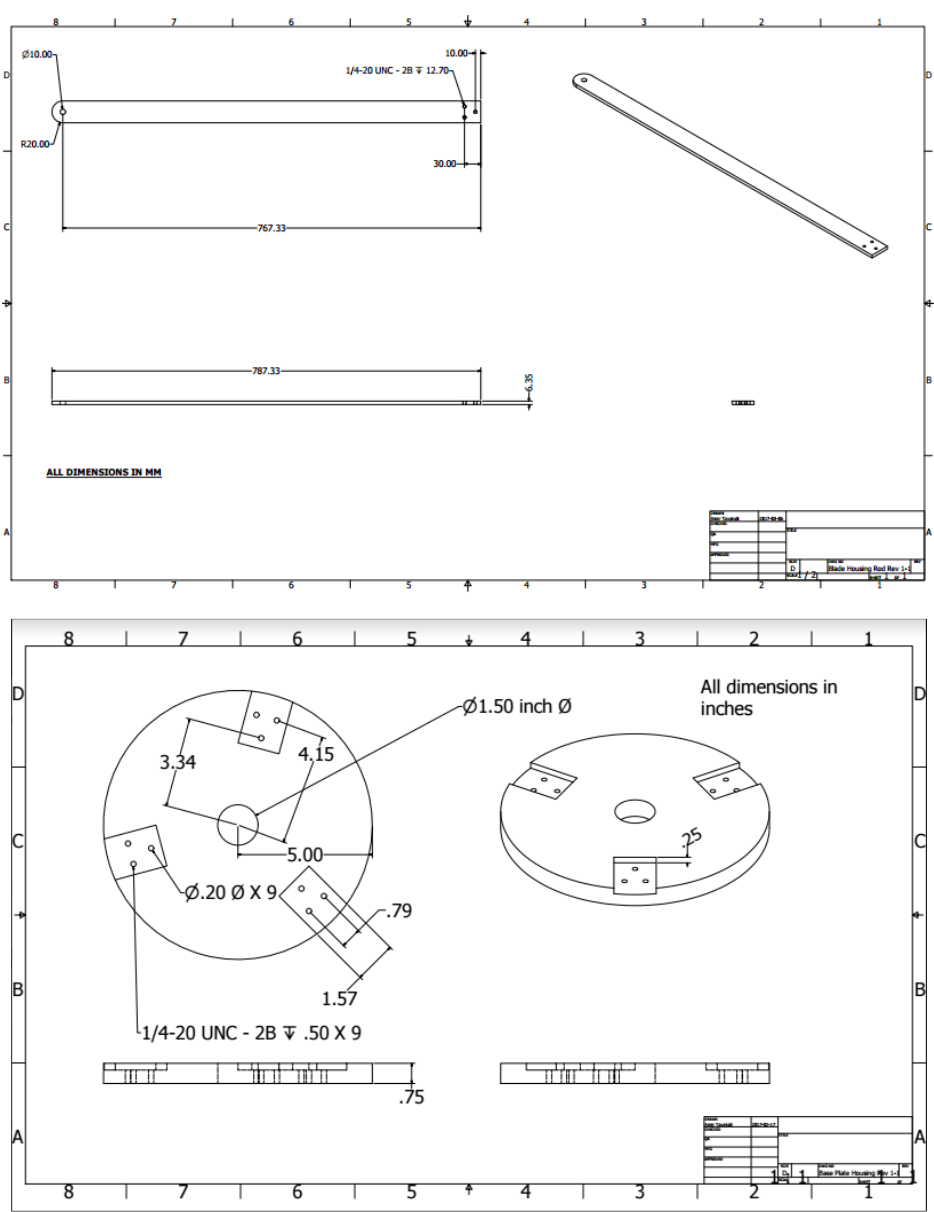

Figure C2. (a) Top and bottom hub final drawing, (b) Hub linkages' rods 


\section{MeMASTER-CARR.}

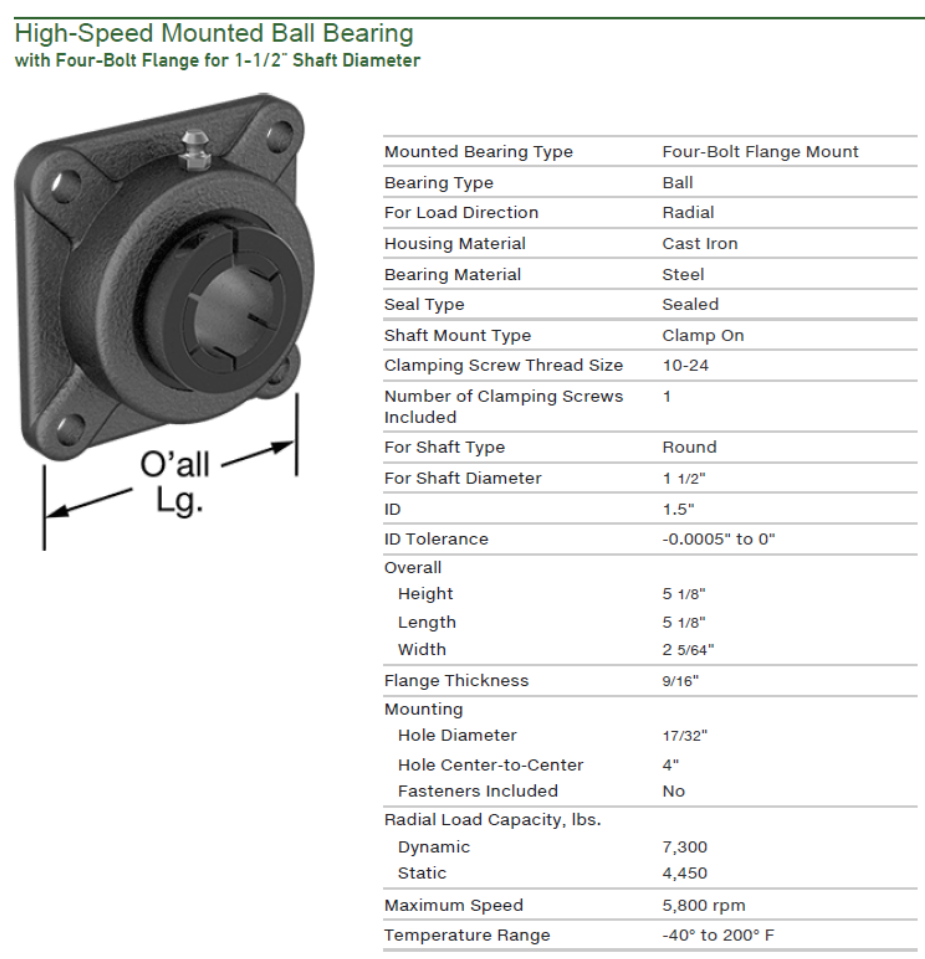

Figure $\mathrm{C} 3$. Main shaft bearing $\mathrm{CAD}$ and specifications.

\section{C1: PMSG specifications}

Fisher\&Paykel
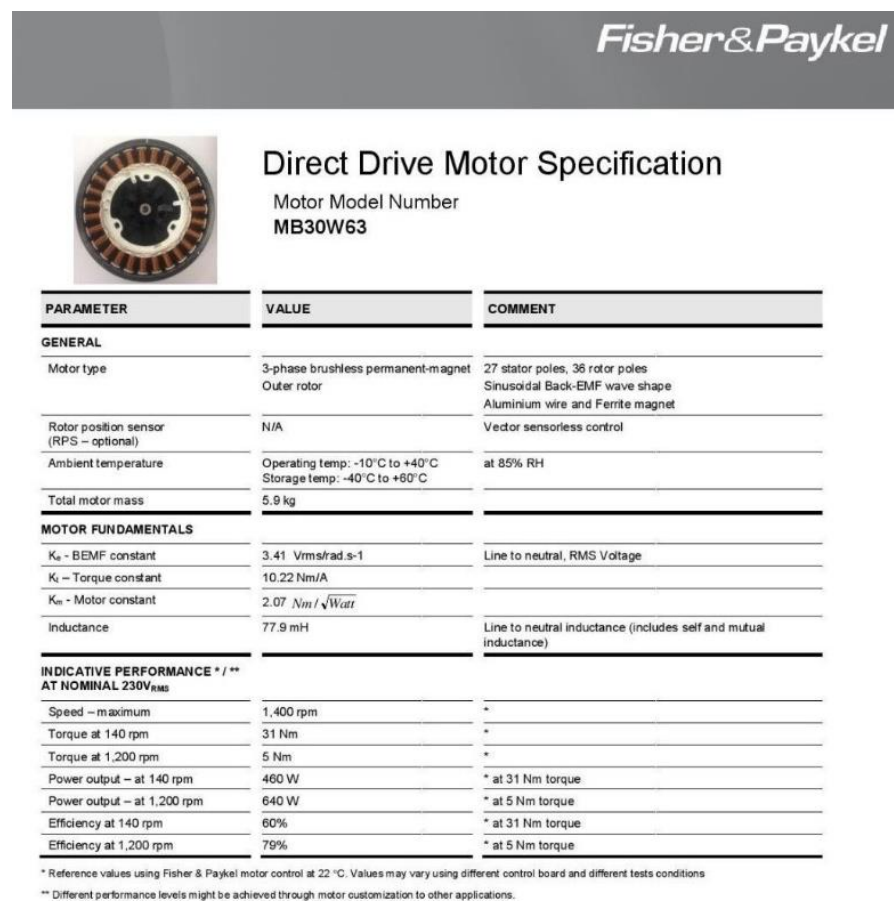


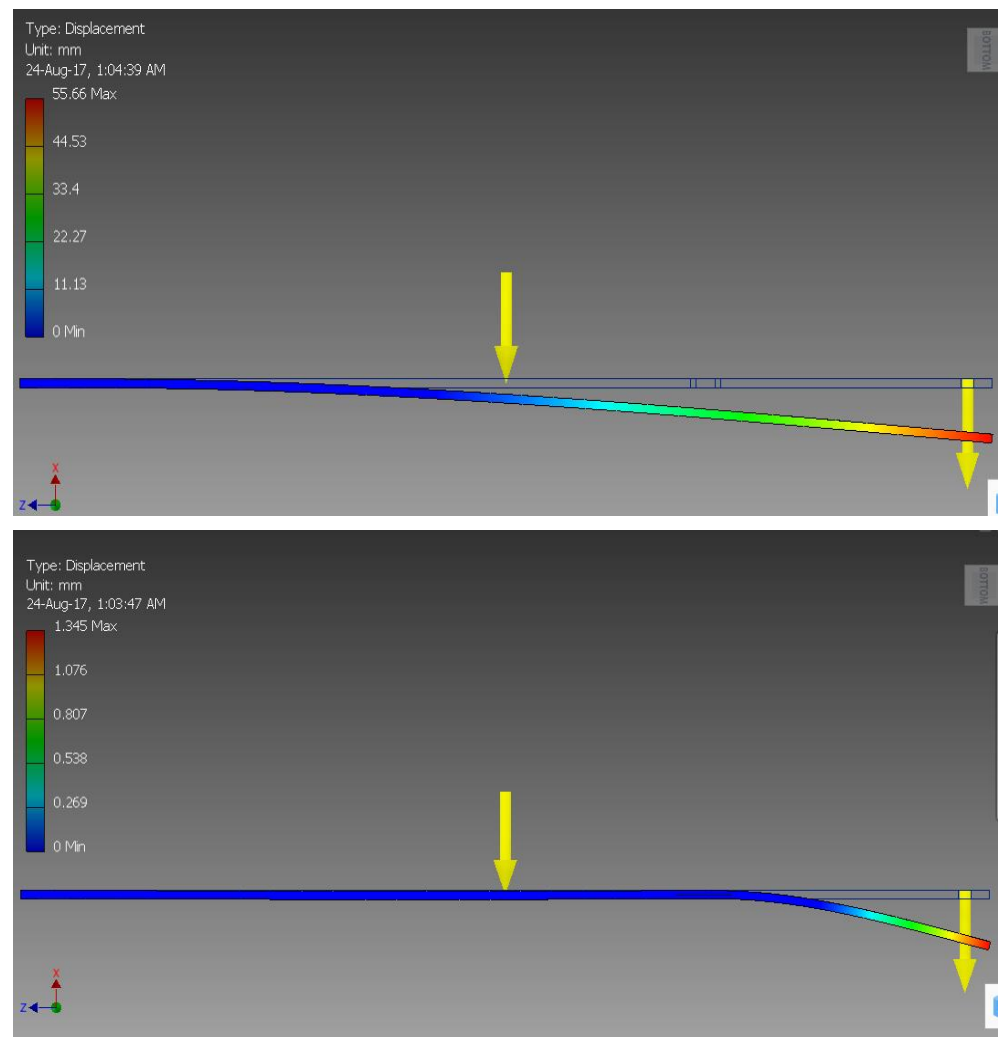

Figure C4 (a) bending due to blade weight, (b) bending after adding blade support structure
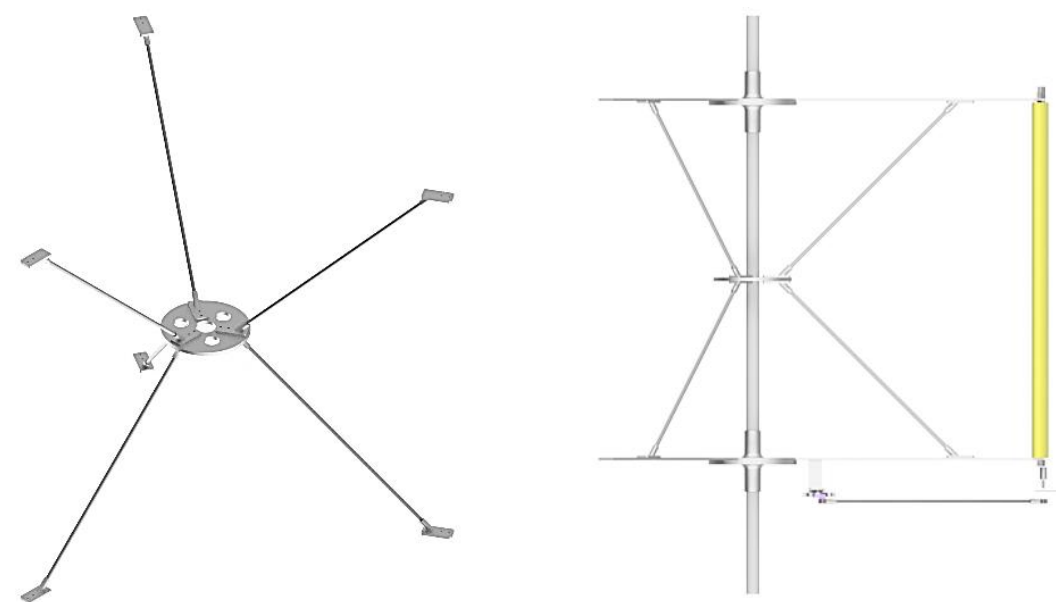

Figure C5 Blade support structure. 
Table C1. HG37D670WE12 - 052FH motor characteristics

\begin{tabular}{ll}
\hline Nominal Voltage: & $7.2 \mathrm{~V}$ \\
No-load speed: & $160 \mathrm{rpm}$ \\
Reduction: & $1: 52$ \\
Torque: & $100 \mathrm{oz}-\mathrm{in}(7.2 \mathrm{~kg}-\mathrm{cm})$ \\
Weight: & $4.7 \mathrm{oz}$ \\
Motor cables: & 12 "20AWG \\
Quadrature encoder cables: & 12 "20AWG $(+5 \mathrm{~V}, \mathrm{Gnd}, \mathrm{A}, \mathrm{B})$ \\
Resolution: & 624 pulses per revolution of the axis \\
Diameter of the shaft: & $6 \mathrm{~mm}$ \\
Current without load: & $300 \mathrm{~mA}$ \\
Maximum current (rotor blocked): & $2.0 \mathrm{~A}$ \\
\hline
\end{tabular}

\section{C2: Slip ring specifications}
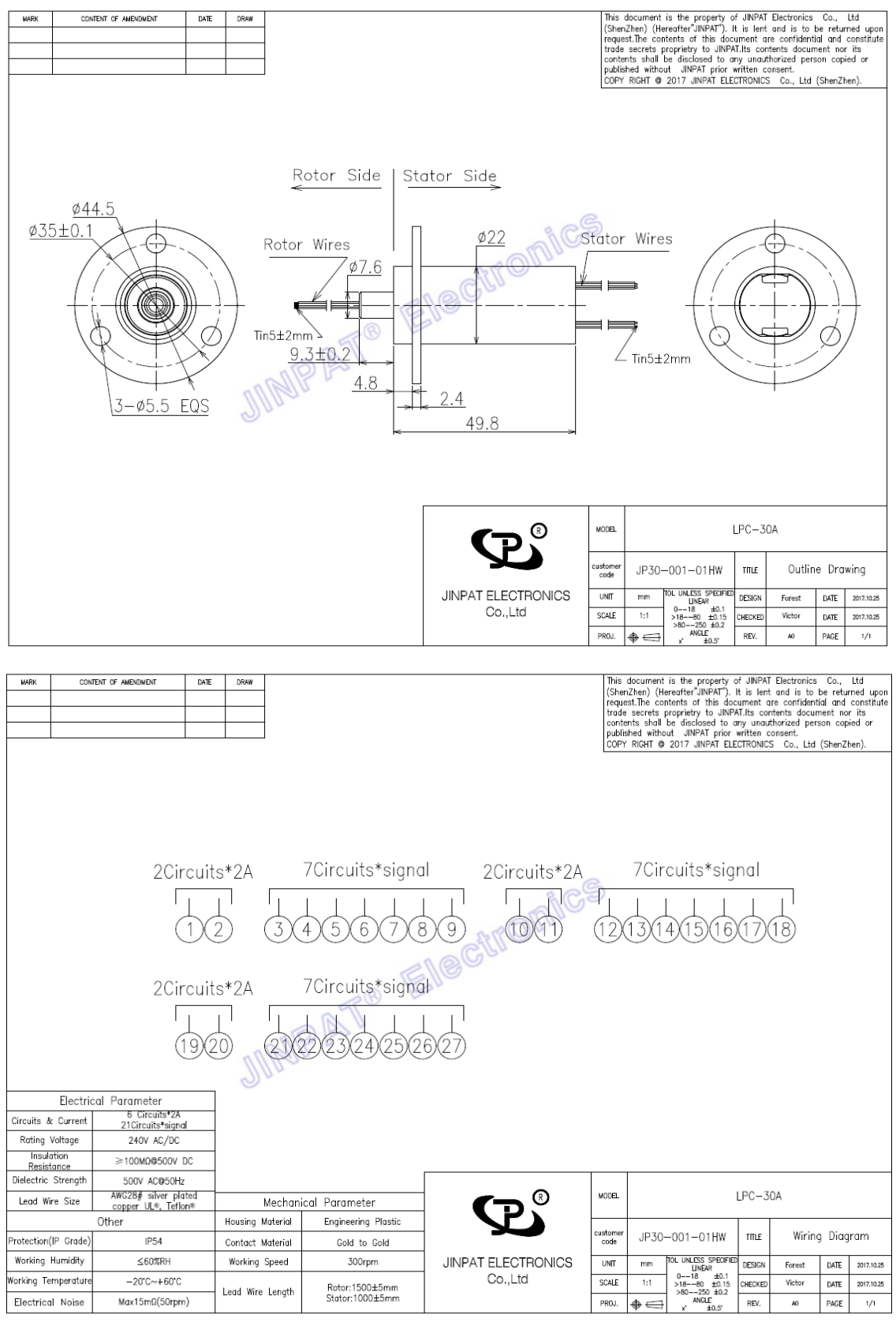


\section{C3: Initialization and main loop of the proposed Hybrid controller}

\section{a. Initialization}

The system is initialized by using limit switch for a potentiometer sensor calibration. The main steps for initialization are:

- Digital signal is given to each motor driver where one of the pins is false and the other is true in order to rotate in one direction and the Pulse Width Modulation (PWM) is determined by the user in order to control the speed of the motor.

- The motor keeps rotating the blade until it presses on the corresponding limit switch. Next, the program stops the motor and then records the measured blade angle as $-6^{\circ}$.

- Next step, the digital signal sent to each motor driver is reversed causing reversing the motor direction.

- The motor keeps rotating the blade until it presses on the corresponding limit switch. Next, the program stops the motor and then records the measured blade angle as $+6^{\circ}$.

\section{b. Main loop implementation}

In Fig. (6.13), the main loop can be described by following steps:

- The program measures the current rotor angle, and accordingly, sets to each blade the corresponding set-point from a predefined lookup table.

- Next, each blade angle is measured by potentiometer, filtered and fed to the Hybrid controller.

- PID controller measures the error and based on the tuned PID gains and saturation limits, the corresponding a PWM signal is calculated.

- MLP-ANN Controller also receives the error signal, its derivative, rotor position $(\theta)$, and reference pitch angle for producing an MLP-ANN PWM value.

Finally, both PWM values of PID and MLP-ANN controllers are weighted by $w_{1}$ and $w_{2}$ parameters, respectively, which are summed to produce a final PWM value which is sent to each motor driver. 